\title{
Asymptotics of Cholesky GARCH models and time-varying conditional betas
}

\author{
Serge Darolles a, Christian Francq ${ }^{\mathrm{b}}$, Sébastien Laurent ${ }^{\mathrm{c}, *}$ \\ a Université Paris-Dauphine, PSL Research University, CNRS, UMR 7088, DRM, Finance, France \\ b CREST, ENSAE, Université Paris-Saclay and University Lille 3, France \\ ${ }^{\mathrm{c}}$ Aix-Marseille University (Aix-Marseille School of Economics), CNRS \& EHESS, Aix-Marseille Graduate School of Management-IAE, France
}

\begin{abstract}
A B S T R A C T
This paper proposes a new model with time-varying slope coefficients. Our model, called CHAR, is a Cholesky-GARCH model, based on the Cholesky decomposition of the conditional variance matrix intro-duced by Pourahmadi (1999) in the context of longitudinal data. We derive stationarity and invertibility conditions and prove consistency and asymptotic normality of the Full and equation-by-equation QML estimators of this model. We then show that this class of models is useful to estimate conditional betas and compare it to the approach proposed by Engle (2016). Finally, we use real data in a portfolio and risk management exercise. We find that the CHAR model outperforms a model with constant betas as well as the dynamic conditional beta model of Engle (2016).
\end{abstract}

JEL classification: C13; C32; C53; C58

Keywords: Multivariate-GARCH; Conditional betas; Covariance

\section{Introduction and motivations}

The estimation of linear models on time series data is common in finance, especially in the context of factor models. The standard practice is to assume that the slope coefficients are constant over time. However, wrongly assuming the constancy of the parameters leads to misspecified models and potentially wrong financial decisions. For example, testing an asset pricing model such as the Capital Asset Pricing Model (CAPM) (Sharpe, 1964) with constant regression parameters may lead to a wrong rejection of the model if the parameters are changing over time. Similarly, attempting to evaluate the performance of an active management fund using the Sharpe Style Analysis Model (Sharpe, 1992) with constant parameters may lead to an over-valuation of the manager's skills. It is therefore important to develop simple statistical approaches capturing all the dynamic aspects of the financial series.

Recently, Engle (2016) proposed a new model called Dynamic Conditional Beta (DCB in short) to obtain time-varying slope coefficients by extending Bollerslev et al. (1998) to the case of more than

\footnotetext{
* Corresponding author.

E-mail addresses: serge.darolles@dauphine.fr (S. Darolles), christian.francq@univ-lille3.fr (C. Francq),sebastien.laurent@univ-amu.fr (S. Laurent).
}

one explanatory variable (or factor). ${ }^{1}$ Assuming joint conditional normality of the variables appearing in the model (i.e., dependent and independent variables), Engle (2016) shows how to recover indirectly the time-varying slope coefficients of the independent variables using an estimate of the full conditional covariance matrix. In his application, he relies on a DCC model to obtain the conditional betas of the Fama-French three factor model. While this approach is easy to implement, testing and imposing the constancy of the conditional betas is cumbersome. Furthermore, identifying the variables determining the evolution of the betas is impossible because conditional betas are not modeled directly but recovered afterwards by a non-linear transformation of the elements of the estimated conditional covariance matrix.

In this paper, we propose a different approach essentially based on a natural orthogonalization of the observed time series, initially proposed by Pourahmadi (1999) in the context of longitudinal data. We first study the Cholesky-GARCH model (CHAR in short), a model specifying directly the dynamics of time varying slope coefficients. We extend the work of Pourahmadi (1999) by considering time varying slope coefficients that depend on their lagged values and past shocks. We derive stationarity and invertibility

\footnotetext{
1 Maheu and Shamsi (2016) propose a nonparametric DCB model in a Bayesian framework.
} 
conditions and prove consistency and asymptotic normality of the Full and equation-by-equation (EbE) QML estimators of this model. Our approach is very flexible because it allows to impose easily the constancy of some of the conditional betas. Furthermore, unlike conditional correlations, conditional betas can take any real values, which allows the use of general specifications.

We compare the numerical properties of the two estimators using a Monte-Carlo simulation study. Our results suggest that both the Full and EbE QML deliver satisfactory results in the sense that biases are very small for all parameters. In an empirical application, we show that this class of models is useful to estimate conditional betas in the context of the Fama-French three factor model. We consider four competing models to obtain one-step-ahead forecasts of the three conditional betas, i.e., two DCB models based on a CCC-GARCH $(1,1)$ or a DCC-GARCH $(1,1)$, our proposed model with time-varying betas and a special case of this model where the constancy of the betas is imposed. We use these forecasts to build tracking portfolios for the 12 US industry portfolios (Data are from Ken French's web site and cover the period spanning from February 1994 to August 2016). We rely on two loss functions (i.e., mean square error or mean absolute deviation) to quantify the magnitude of the tracking errors (i.e., difference between the observed returns and the tracking portfolios) of the four models and like Laurent et al. (2012) and Amado and Teräsvirta (2014) apply the Model Confidence Set (MCS) test of Hansen et al. (2011) to discriminate between them. We find that on the whole forecasting period (i.e., 2010-2016), our proposed model with timevarying betas belongs to the set of superior models in all cases while the DCB based on the DCC model belongs to this set in only 3 and 2 cases, when relying respectively on the MSE and MAD loss functions. Interestingly, the DCB-CCC model and the CHAR model with constant betas never show up in the MCS. We also show that our proposed model has forecasted betas that are much smoother than those obtained with the DCB-DCC model, which translates into smaller transaction costs.

The paper is organized as follows. In Section 2, we review the DCB model of Engle (2016) and present the CHAR model. Stationarity conditions for the CHAR model are studied in Section 3. The Full and EbE QML estimators of this model are presented in Section 4 as well as invertibility conditions. Monte Carlo simulation results are reported in Section 5 and an application in Section 6. Finally, Section 7 concludes.

\section{Direct versus indirect specification of conditional betas}

Before studying in more detail the properties of our proposed model, let us present some notation and a competing approach delivering time-varying slope coefficients. Let $\boldsymbol{\epsilon}_{t}=\left(\epsilon_{1 t}, \ldots, \epsilon_{m t}\right)^{\prime}$ be a vector of $m \geq 2$ returns with mean zero and satisfying a general volatility model of the form

$\boldsymbol{\epsilon}_{t}=\Sigma_{t}^{1 / 2}\left(\boldsymbol{\vartheta}_{0}\right) \boldsymbol{\eta}_{t}, \quad t=1, \ldots, n$,

where $\left(\boldsymbol{\eta}_{t}\right)$ is a sequence of independent and identically distributed (i.i.d.) random vectors with zero mean and identity covariance matrix, and

$\boldsymbol{\Sigma}_{t}=\boldsymbol{\Sigma}_{t}\left(\boldsymbol{\vartheta}_{0}\right)=\boldsymbol{\Sigma}\left(\boldsymbol{\epsilon}_{t-1}, \boldsymbol{\epsilon}_{t-2}, \ldots ; \boldsymbol{\vartheta}_{0}\right)$

is almost surely a positive definite $m \times m$ matrix, parametrized by a $d$-dimensional parameter $\vartheta_{0}$ and depending on the information $\mathcal{F}_{t-1}$ generated by the past values of $\boldsymbol{\epsilon}_{t}$. Assuming that (2.1) admits a non anticipative stationary solution (the stationarity conditions are discussed in the sequel), $\Sigma_{t}$ is the conditional variancecovariance matrix of $\epsilon_{t}$.

Multivariate GARCH (MGARCH) models are very well suited for empirical applications needing an estimate or a forecast of $\Sigma_{t}$ (see
Bauwens et al., 2006; Silvennoinen and Teräsvirta, 2009 for a survey of MGARCH models). A natural example is the computation of the value-at-risk of a portfolio, when the portfolio's composition is observable and time-varying (see e.g., Francq and Zakoïan, 2017). However, MGARCH models are also used in financial applications involving a non-linear transformation of $\Sigma_{t}$. For instance, in the mean-variance framework (see e.g., Markowitz, 1952 in a static case), the optimal weights of a portfolio depend on an estimate of $\Sigma_{t}^{-1}$. Another example is the estimation of conditional betas using the DCB model of Engle (2016) presented in the next subsection. In this paper, we advocate the use of a model that specifies directly the dynamics of the conditional betas instead of $\Sigma_{t}$.

\subsection{Indirect specification of dynamic conditional betas}

In some empirical finance applications, it is necessary to give a special role to one of the asset embedded in the vector $\boldsymbol{\epsilon}_{t}$, for example when a particular return series, say $y_{t}=\epsilon_{m t}$, is regressed on the other components of the vector $\epsilon_{t}$, i.e., $\mathbf{x}_{t}=$ $\left(\epsilon_{1 t}, \ldots, \epsilon_{m-1, t}\right)^{\prime}$. The coefficients of the multivariate regression of $y_{t}$ on $\mathbf{x}_{t}$ are usually called betas. To obtain time-varying betas, Engle (2016) assumes that $\boldsymbol{\epsilon}_{t}=\left(\mathbf{x}_{t}, y_{t}\right)^{\prime}$ has a multivariate normal distribution (conditional on $\mathcal{F}_{t-1}$ ) with conditional covariance $\Sigma_{t}$, i.e.,

$$
\left(\begin{array}{l}
\mathbf{x}_{t} \\
y_{t}
\end{array}\right) \mid \mathcal{F}_{t-1} \sim \mathcal{N}\left(\left(\begin{array}{c}
\mathbf{0}_{m-1} \\
0
\end{array}\right),\left(\begin{array}{cc}
\boldsymbol{\Sigma}_{x x, t} & \boldsymbol{\Sigma}_{x y, t} \\
\boldsymbol{\Sigma}_{y x, t} & \Sigma_{y y, t}
\end{array}\right)\right),
$$

where subscripts represent natural partitions. Engle (2016) uses the fact that the conditional distribution of $y_{t}$ on $\mathbf{x}_{t}$ is

$y_{t} \mid \mathbf{x}_{t} \sim \mathcal{N}\left(\boldsymbol{\Sigma}_{y x, t} \boldsymbol{\Sigma}_{x x, t}^{-1} \mathbf{x}_{t}, \Sigma_{y y, t}-\boldsymbol{\Sigma}_{y x, t} \boldsymbol{\Sigma}_{x x, t}^{-1} \boldsymbol{\Sigma}_{x y, t}\right)$

to derive an estimate of the conditional betas. Indeed, estimates of the time-varying coefficients of the regression of $y_{t}$ on $\mathbf{x}_{t}$ can be recovered from $\Sigma_{t}$ by the formula $\Sigma_{x x, t}^{-1} \Sigma_{x y, t}$ or $\Sigma_{x y, t} / \Sigma_{x x, t}$ if there is only one explanatory variable (like in Bollerslev et al., 1998). Engle (2016) advocates the use of a Dynamic Conditional Correlation (DCC) model to estimate $\Sigma_{t}$, but any multivariate GARCH model can be used. The DCC approach relies on the following decomposition of $\Sigma_{t}$ :

$\Sigma_{t}=\boldsymbol{D}_{t} \boldsymbol{R}_{t} \boldsymbol{D}_{t}$

where $\boldsymbol{D}_{t}$ and $\boldsymbol{R}_{t}$ are respectively a diagonal matrix with the conditional volatilities of $\epsilon_{t}$ and its conditional correlation matrix. Matrix $\boldsymbol{D}_{t}$ is typically modeled using $m$ univariate GARCH models on $\boldsymbol{\epsilon}_{t}$ and $\boldsymbol{R}_{t}$ using a scalar BEKK specification on the devolatilized series $\boldsymbol{D}_{t}^{-1} \boldsymbol{\epsilon}_{t}$ (which is transformed afterwards to get a correlation matrix). The Constant Conditional Correlation (CCC) model of Bollerslev (1990) is obtained by setting $\boldsymbol{R}_{t}=\boldsymbol{R}$.

\subsection{Direct specification of dynamic conditional betas}

The specificity of the previous approach is to deduce the conditional betas from an estimate of $\Sigma_{t}$. Our approach is radically different because it allows to directly model these conditional betas. It is based on a natural orthogonalization of $\epsilon_{t}$, initially proposed by Pourahmadi (1999) in the case of longitudinal data. We extend his work by allowing the slope coefficients to vary over time with an autoregressive structure.

We follow Tsay (2010, Chapter 7) and introduce recursively the orthogonal factors obtained from $\epsilon_{t}$. Let $\ell_{21, t}$ be the time-varying coefficient in the regression of $\epsilon_{2 t}$ on $v_{1 t}:=\epsilon_{1 t}$, conditional on $\mathcal{F}_{t-1}$. One can write

$\epsilon_{2 t}=\ell_{21, t} v_{1 t}+v_{2 t}=\beta_{21, t} \epsilon_{1 t}+v_{2 t}$, 
where $\beta_{21, t}=\ell_{21, t} \in \mathcal{F}_{t-1}$ is the conditional beta in the regression of $\epsilon_{2 t}$ on $\epsilon_{1 t}$, and $v_{2 t}$ is orthogonal to $\epsilon_{1 t}$ conditionally on $\mathcal{F}_{t-1}$. More generally, we have

$\epsilon_{i t}=\sum_{j=1}^{i-1} \ell_{i j, t} v_{j t}+v_{i t}=\sum_{j=1}^{i-1} \beta_{i j, t} \epsilon_{j t}+v_{i t}, \quad$ for $i=2, \ldots, m$,

where $v_{i t}$ is uncorrelated to $v_{1 t}, \ldots, v_{i-1, t}$, and thus uncorrelated to $\epsilon_{1 t}, \ldots, \epsilon_{i-1, t}$, conditionally on $\mathcal{F}_{t-1}$. In particular, we obtain

$\beta_{31, t}=\ell_{31, t}-\ell_{32, t} \ell_{21, t}, \quad \beta_{32, t}=\ell_{32, t}$.

In matrix form, (2.3) can be written

$\boldsymbol{\epsilon}_{t}=\boldsymbol{L}_{t} \boldsymbol{v}_{t} \quad$ and $\quad \boldsymbol{B}_{t} \boldsymbol{\epsilon}_{t}=\boldsymbol{v}_{t}$,

where $\boldsymbol{L}_{t}$ and $\boldsymbol{B}_{t}=\boldsymbol{L}_{t}^{-1}$ are lower unitriangular (i.e., lower triangular with 1 on the diagonal) matrices, with $\ell_{i j, t}$ (resp. $-\beta_{i j, t}$ ) at the row $i$ and column $j$ of $\boldsymbol{L}_{t}$ (resp. $\boldsymbol{B}_{t}$ ) for $i>j$. For instance, for $m=3$, we have

$\boldsymbol{L}_{t}=\left(\begin{array}{ccc}1 & 0 & 0 \\ \ell_{21, t} & 1 & 0 \\ \ell_{31, t} & \ell_{32, t} & 1\end{array}\right)$ and $\boldsymbol{B}_{t}=\left(\begin{array}{ccc}1 & 0 & 0 \\ -\beta_{21, t} & 1 & 0 \\ -\beta_{31, t} & -\beta_{32, t} & 1\end{array}\right)$.

The vector $\boldsymbol{v}_{t}=\left(v_{1 t}, \ldots, v_{m t}\right)^{\prime}$ can be interpreted as a vector of orthogonal factors, whose covariance matrix $\boldsymbol{G}_{t}$ is therefore diagonal but not necessarily constant over time, i.e., $\boldsymbol{G}_{t}=$ $\operatorname{diag}\left(g_{1, t}, \ldots, g_{m, t}\right)$ with $g_{i t}>0$ a.s., for $i=1, \ldots, m$ (since $\Sigma_{t}$ is positive definite) ${ }^{2}$

We end up with the Cholesky decomposition of Pourahmadi (1999), i.e.,

$\boldsymbol{\Sigma}_{t}\left(\boldsymbol{\vartheta}_{0}\right)=\Sigma_{t}=\operatorname{Var}\left(\boldsymbol{L}_{t} \boldsymbol{v}_{t}\right)=\boldsymbol{L}_{t} \boldsymbol{G}_{t} \boldsymbol{L}_{t}^{\prime}$

which is an alternative to (2.2). Using (2.5), (2.1) can be rewritten as

$\boldsymbol{\epsilon}_{t}=\boldsymbol{L}_{t} \boldsymbol{G}_{t}^{1 / 2} \boldsymbol{\eta}_{t}$

where $\left(\boldsymbol{\eta}_{t}\right)$ is defined as above.

Interestingly, when $i=m$, (2.3) corresponds to the regression of $y_{t}$ on $\mathbf{x}_{t}$ with time-varying coefficients (in the case of demeaned series), i.e., $\epsilon_{m t}=\sum_{j=1}^{m-1} \beta_{m j, t} \epsilon_{j t}+v_{m t}$, that are directly comparable with those obtained indirectly with the DCB model of Engle (2016). However, our approach allows to directly specify a model for the $\beta_{i j, t}$ coefficients without having to impose any constraint apart from the stationarity constraint discussed in Section 3.

As pointed out by a referee, there is a resemblance between (2.4) and the OGARCH model of Alexander and Chibumba (1997) and Alexander (2001) and its generalizations (see van der Weide, 2002; Lanne and Saikkonen, 2007; Fan et al., 2008; Boswijk and van der Weide, 2011 among others). The OGARCH model can be expressed as $\boldsymbol{\epsilon}_{t}=\boldsymbol{Z} \boldsymbol{v}_{t}^{*}$, with $\boldsymbol{Z}=\boldsymbol{P} \boldsymbol{E}^{1 / 2}$ an orthogonal matrix, where $\boldsymbol{P}$ is a matrix of orthogonal eigenvectors and $\boldsymbol{E}$ a diagonal matrix containing the eigenvalues of the unconditional covariance matrix of $\boldsymbol{\epsilon}_{t}$. The components of the vector $\boldsymbol{v}_{t}^{*}$ are orthogonal (and heteroscedastic) factors corresponding to the principal components of $\epsilon_{t}$. Therefore, like the CHAR model, the OGARCH model assumes that the observed data $\epsilon_{t}$ can be linearly transformed into a set of uncorrelated factors.

However, the OGARCH model, as its extensions, specifies the dynamics of the conditional covariance matrix via a factor decomposition and the modeling of the conditional variance of these factors. Therefore, unlike the CHAR model, the OGARCH model

\footnotetext{
2 We follow the R language convention that $\operatorname{diag}(\boldsymbol{v})$ is a diagonal matrix with the vector $\boldsymbol{v}$ on the diagonal, and $\operatorname{diag}(\boldsymbol{M})$ is the vector of the diagonal of $\boldsymbol{M}$, when $\boldsymbol{M}$ is a square matrix.
}

does not directly specify the dynamics of conditional betas, which is the primary aim of this paper (although conditional betas can be recovered from the estimated conditional covariance matrix using the DCB approach of (Engle, 2016)).

Importantly, the two sets of factors we obtain with the CHAR and OGARCH models differ for two reasons:

1. the elements of $\boldsymbol{v}_{t}$ are the residuals of linear models with time-varying slope coefficients for the CHAR model while they are principal components for the OGARCH model;

2. the projection matrix $\boldsymbol{Z}$ is constant in the OGARCH model while $\boldsymbol{L}_{t}$ and $\boldsymbol{B}_{t}$ are time-varying in the CHAR model. If the true rotation matrix $\boldsymbol{Z}$ is not constant over time, wrongly assuming it to be constant leads to factors that at best unconditionally uncorrelated. Note that the same comment applies to the GOGARCH model, for which $\boldsymbol{Z}$ is assumed to be invertible and not necessarily orthogonal.

Recently, Noureldin et al. (2014) proposed a model, called RARCH, for which the factors are uncorrelated both conditionally and unconditionally, and the factor loadings are time-varying. However, unlike the CHAR model, the RARCH does not directly specify the dynamics of conditional betas.

\subsection{A general parameterization of the CHAR model}

An attractive feature of the Cholesky-GARCH (CHAR) model is that the dynamics of $\epsilon_{t}$ can be defined by specifying successively the dynamics of the vector $\boldsymbol{v}_{t}$ of the orthogonal factors and the dynamics of the vector $\boldsymbol{\ell}_{t}=\operatorname{vech}^{0} \boldsymbol{L}_{t}$ of the subdiagonal elements of $\boldsymbol{L}_{t}$, or alternatively the dynamics of $\boldsymbol{\beta}_{t}=-\operatorname{vech}^{0} \boldsymbol{B}_{t} .^{3}$

We illustrate our general framework by first considering, for the dynamics of this specification, the Extended Constant Conditional correlation GARCH model studied by Jeantheau (1998), Ling and McAleer (2003), He and Teräsvirta (2004), Aue et al. (2009), Francq and Zakoïan (2010) and Pedersen (2017), among others. This model assumes that

$\mathbf{g}_{t}=\boldsymbol{\omega}_{0}+\sum_{i=1}^{q} \boldsymbol{A}_{0 i} \boldsymbol{v}_{t-i}^{2}+\sum_{j=1}^{p} \boldsymbol{B}_{0 j} \mathbf{g}_{t-j}$

where $\boldsymbol{g}_{t}=\left(g_{1, t}, \ldots, g_{m, t}\right)^{\prime}$. To guarantee strict positivity of the components of $\mathbf{g}_{t}$, it is assumed that $\omega_{0}$ is a vector of strictly positive coefficients, and that $\boldsymbol{A}_{0 i}$ and $\boldsymbol{B}_{0 j}$ are matrices of positive coefficients. Model (2.6) is an extension of the initial model introduced by Bollerslev (1990) for which the matrices $\boldsymbol{A}_{0 i}$ and $\boldsymbol{B}_{0 j}$ are assumed to be diagonal. The variances defined by (2.6) are functions of squared returns, and thus the model is not able to take into account the leverage effect commonly observed on financial series of daily returns, i.e., the fact that negative returns tend to have a higher impact on the future volatility than positive returns of the same magnitude. An extension of (2.6) allowing asymmetric responses of positive and negative past returns on the future volatility is the Asymmetric GARCH (AGARCH) model studied by McAleer et al. (2009) and Francq and Zakoïan (2012), among others. This model can be seen as a multivariate extension of the GJR model (Glosten et al., 1993). More precisely, we assume that

$\mathbf{g}_{t}=\boldsymbol{\omega}_{0}+\sum_{i=1}^{q}\left\{\boldsymbol{A}_{0 i,+} \boldsymbol{v}_{t-i}^{2+}+\boldsymbol{A}_{0 i,-} \boldsymbol{v}_{t-i}^{2-}\right\}+\sum_{j=1}^{p} \boldsymbol{B}_{0 j} \mathbf{g}_{t-j}$,

where

$\boldsymbol{v}_{t}^{2+}=\left(\left\{v_{1 t}^{+}\right\}^{2}, \ldots,\left\{v_{m t}^{+}\right\}^{2}\right)^{\prime}, \quad \boldsymbol{v}_{t}^{2-}=\left(\left\{v_{1 t}^{-}\right\}^{2}, \ldots,\left\{v_{m t}^{-}\right\}^{2}\right)^{\prime}$

\footnotetext{
${ }^{3}$ vech $^{0}$ denotes the operator stacking the sub-diagonal elements of a square
} matrix. 
with $x^{+}=\max (x, 0)=(-x)^{-}$. To guarantee strict positivity of the components of $\boldsymbol{g}_{t}$, the parameters $\boldsymbol{\omega}_{0}$ and $\boldsymbol{B}_{0 j}$ are as in (2.6), and $\boldsymbol{A}_{0 i,+}, \boldsymbol{A}_{0 i,-}$ are matrices of positive coefficients.

Note that for the above-mentioned traditional multivariate GARCH processes, a model of the form (2.6) or (2.7) is applied to the vector of the individual volatilities of $\epsilon_{t}$ in a first step. In a second step, the model is completed by specifying either a fixed or a time-varying conditional correlation, leading to the classification of the CCC and DCC-GARCH models (see Bauwens et al. (2006), Silvennoinen and Teräsvirta (2009), Francq and Zakoïan (2010, Chapter 11), Tsay (2010, Chapter 7), Bauwens et al. (2012) for general references). Models for the conditional correlation are complicated, in particular because the modeling of conditional correlations requires imposing strong restrictions on the type of dynamics and in some cases the use of a non-linear transformation to recover the conditional correlations from quasi conditional correlations. This is the reason why no complete asymptotic theory is currently available for estimating DCC-GARCH models.

The Cholesky-GARCH approach avoids specifying a correlation structure but requires specifying how conditionally orthogonal factors $\boldsymbol{v}_{t}$ are obtained from $\boldsymbol{\epsilon}_{t}$ as well as the dynamics of their conditional variances. Moreover, the Cholesky-GARCH approach presents the strong advantage that the vector $\ell_{t}$, of size $m_{0}=$ $m(m-1) / 2$, is not constrained. One can thus consider a model of the form

$\boldsymbol{\ell}_{t}=\boldsymbol{c}_{0}\left(\boldsymbol{v}_{t-1}, \ldots, \boldsymbol{v}_{t-r}, \mathbf{g}_{t-1}^{1 / 2}, \ldots, \mathbf{g}_{t-r}^{1 / 2}\right)+\sum_{j=1}^{s} \boldsymbol{c}_{0 j} \boldsymbol{\ell}_{t-j}$,

where $\boldsymbol{c}_{0}$ is a general measurable function from $\mathbb{R}^{m} \times \cdots \times \mathbb{R}^{m}$ to $\mathbb{R}^{m_{0}}$ and where the $\boldsymbol{C}_{0 j}$ 's are $m_{0} \times m_{0}$ matrices of coefficients, in particular not constrained to be positive. ${ }^{4}$ Alternatively to (2.8), one could specify a dynamic model directly on the beta's:

$\boldsymbol{\beta}_{t}=\boldsymbol{c}_{0}\left(\boldsymbol{v}_{t-1}, \ldots, \boldsymbol{v}_{t-r}, \mathbf{g}_{t-1}^{1 / 2}, \ldots, \boldsymbol{g}_{t-r}^{1 / 2}\right)+\sum_{j=1}^{s} \boldsymbol{c}_{0 j} \boldsymbol{\beta}_{t-j}$.

Stationarity conditions are derived in Section 3 for Models (2.7)-(2.8) and (2.7)-(2.9) while consistency and asymptotic normality of the Full QMLE of these models are proved under very general assumptions in Section 4.

\subsection{Particular submodels}

To get more explicit conditions for stationarity and invertibility of the CHAR model as well as consistency and asymptotic normality of the Full and EbE QMLE, we consider particular examples of the general specification (2.7)-(2.9).

For the conditional variance of the factors, we first assume that

$$
\begin{aligned}
g_{i, t}= & \omega_{0 i}+\gamma_{0 i+}\left(\epsilon_{1, t-1}^{+}\right)^{2}+\gamma_{0 i-}\left(\epsilon_{1, t-1}^{-}\right)^{2} \\
& +\sum_{k=2}^{i} \alpha_{0 i}^{(k)} v_{k, t-1}^{2}+b_{0 i} g_{i, t-1}
\end{aligned}
$$

for $i=1, \ldots, m$, with positivity constraints on the coefficients. By convention, any term of the form $\sum_{k=2}^{i}$ vanishes when $i<$ 2 . Note that the volatility of the $i$ th factor can depend on the past values of the factors of indices $k \leq i$. This model allows volatility spillovers between the factors. Note also that, although the factors are, by definition, conditionally uncorrelated, and thus marginally uncorrelated when second-order moments exist, they are not independent.

\footnotetext{
4 Osiewalski and Pajor (2009) use a multivariate volatility model based on the Cholesky decomposition (2.5) in which the elements of $\boldsymbol{\ell}_{t}$ have structure that resembles stochastic volatility model (i.e., with a latent AR(1) structure).
}

It has to be underlined that the possible asymmetry of $g_{i t}$ is taken into account by a function of $\epsilon_{1, t-1}^{+}=v_{1, t-1}^{+}$and $\epsilon_{1, t-1}^{-}$only and not $v_{i, t-1}^{+}$and $v_{i, t-1}^{-}$. This is because we need the existence of derivatives of the volatility with respect to the parameter (see A7 below). To illustrate the issue, let us consider a simple example with $m=2$ and constant beta $\beta_{21, t}=\beta_{21}$. Since we have $v_{2 t}=$ $\epsilon_{2 t}-\beta_{21} \epsilon_{1 t}$, the term $v_{2 t}^{+}$is not derivable with respect to $\beta_{21}$, and thus a volatility of the form

$g_{2, t}=\omega+\gamma_{+}\left(v_{2, t-1}^{+}\right)^{2}+\gamma_{-}\left(v_{2, t-1}^{-}\right)^{2}$

would not be differentiable with respect to the parameter. This would result in a QML estimator optimizing a non differentiable objective function, a situation which is known to cause formidable technical difficulties (see e.g. Chan, 1993).

For the conditional betas, we first adopt the following specification:

$$
\begin{aligned}
\beta_{i j, t}= & \varpi_{0 i j}+\varsigma_{0 i j+} \epsilon_{1, t-1}^{+}+\varsigma_{0 i j-} \epsilon_{1, t-1}^{-} \\
& +\sum_{k=2}^{i} \tau_{0 i j}^{(k)} v_{k, t-1}+c_{0 i j} \beta_{i j, t-1}
\end{aligned}
$$

for any index $(i, j)$ belonging to the set

$\boldsymbol{T}_{m}=\{(i, j): i=2, \ldots, m$ and $j=1, \ldots, i-1\}$.

This specification allows conditional betas to depend on past shocks on the first $i$ factors as well as the previous conditional beta of the period before. We allow the effect of past shocks to differ depending on their sign but only for the first series because, as in (2.10), we need the existence of derivatives of the betas with respect to the parameter (see the justification just above (2.11)). Note that the purpose of this paper is not to provide a financial explanation for the presence of such non-linearity in conditional betas but to provide a statistical tool to estimate and test the empirical relevance of this feature in the model.

Importantly, no further positivity constraint is required in (2.11) to ensure the positive-definiteness of $\Sigma_{t}$. The same equation could be considered for the $\ell_{i j, t}$ 's but we decided not to study in detail such a specification because the main objective of this paper is to model conditional betas. Note that Tsay (2010) uses a specification where $\ell_{i j, t}$ depends on a constant, $\ell_{i j, t-1}$ and $\epsilon_{i, t-1}$. However, the statistical properties of this model have not been studied yet in the literature.

Eqs. (2.10)-(2.11) define a triangular system in which the dynamics of the $i$ th row depends only on the dynamics of the previous rows.

We also consider a second specification of the CHAR model for which the EbE QMLE can be parallelized, i.e.,

$$
\begin{aligned}
g_{i, t}= & \omega_{0 i}+\gamma_{0 i+}\left(\epsilon_{1, t-1}^{+}\right)^{2}+\gamma_{0 i-}\left(\epsilon_{1, t-1}^{-}\right)^{2}+\alpha_{0 i} v_{i, t-1}^{2}+b_{0 i} g_{i, t-1}, \\
\beta_{i j, t}= & \varpi_{0 i j}+\varsigma_{0 i j+} \epsilon_{1, t-1}^{+}+\varsigma_{0 i j-} \epsilon_{1, t-1}^{-}+\tau_{0 i j} v_{i, t-1} \\
& +\xi_{0 i j} v_{i, t-1} v_{1, t-1}+c_{0 i j} \beta_{i j, t-1} .
\end{aligned}
$$

As in (2.10)-(2.11), the asymmetric effects are introduced via the first factor only. Because of the presence of the product term $v_{i, t-1} v_{1, t-1},(2.13)$ is not a particular case of (2.11). Note that, since the first factor $v_{1, t-1}=\epsilon_{1, t-1}$ is observed, we will show that it is possible to express explicitly $v_{i, t-1} v_{1, t-1}$ as a function of the past observations (an invertibility property that will be further studied in Section 4.1.3).

Introducing a term of the form $v_{i, t-1} v_{j, t-1}$ in (2.14) results in unexplicit invertibility conditions, because the product is related to the past observations by complicated nonlinear recursions. This is however a relevant third model that we considered in the 
empirical application, under the form

$$
\begin{aligned}
\beta_{i j, t}= & \varpi_{0 i j}+\varsigma_{0 i j+} \epsilon_{1, t-1}^{+}+\varsigma_{0 i j-} \epsilon_{1, t-1}^{-}+\tau_{0 i j} v_{i, t-1} \\
& +\xi_{0 i j} v_{i, t-1} v_{j, t-1}+c_{0 i j} \beta_{i j, t-1} .
\end{aligned}
$$

Note that all three specifications (2.10)-(2.11), (2.12)-(2.12) and (2.13)-(2.14) are particular cases of (2.7)-(2.9) and therefore all results obtained for this general class of models also apply to these three specifications (i.e., Theorems 3.1 and 4.1).

Following a referee's suggestion, we consider a fourth model, that is not a subcase of (2.7)-(2.9), i.e.,

$$
g_{i t}=\omega_{0 i}+\sum_{k=1}^{m}\left\{\alpha_{0 i+}^{(k)}\left(\epsilon_{k, t-1}^{+}\right)^{2}+\alpha_{0 i-}^{(k)}\left(\epsilon_{k, t-1}^{-}\right)^{2}\right\}+b_{0 i} g_{i, t-1}
$$

and

$\beta_{i j, t}=\varpi_{0 i j}+\tau_{0 i j} \epsilon_{i, t-1} \epsilon_{j, t-1}+c_{0 i j} \beta_{i j, t-1}$

for $i=1, \ldots, m$. The fact that the volatilities and betas are now functions of past observations, instead of past factors, inhibits finding explicit stationarity conditions but, as will be shown in Section 4.5, renders easier the asymptotic theory of the QMLE.

The main pitfall of dynamic specifications of the elements of a Cholesky decomposition is that the order of the series matters and that most of the times all possible permutations of the series are plausible. ${ }^{5}$ In this case the optimal order can be chosen using information criteria. However, there are cases where a natural ordering is available like in our empirical application. Indeed, we use the CHAR model to estimate a three factors model (à la (Fama and French, 1992, 2004)) with time varying betas. In this case, the last series is necessarily the asset under investigation (i.e., excess returns of an industry portfolio), the first series is likely to be excess returns of the market and the second and third series are the two remaining factors (whose order is chosen using the Schwarz Information Criterion). See Section 6 for more details.

\section{Stationarity of the CHAR model}

The objective of this section is to study the stationarity conditions for the CHAR model. Proofs are gathered in Section 8. Let $\eta_{t}^{2+}=\left(\left\{\eta_{1 t}^{+}\right\}^{2}, \ldots,\left\{\eta_{m t}^{+}\right\}^{2}\right)^{\prime}$ and $\eta_{t}^{2-}=\left(\left\{\eta_{1 t}^{-}\right\}^{2}, \ldots,\left\{\eta_{m t}^{-}\right\}^{2}\right)^{\prime}$, where $\boldsymbol{\eta}_{t}=\left(\eta_{1 t}, \ldots, \eta_{m t}\right)^{\prime}$. For any integer $k$ and any sequence of vectors $\left(\boldsymbol{x}_{t}\right)$, let $\boldsymbol{x}_{t: t-k}=\left(\boldsymbol{x}_{t}^{\prime}, \ldots, \boldsymbol{x}_{t-k}^{\prime}\right)^{\prime}$. With these notations, (2.7) can be rewritten as

$\boldsymbol{z}_{t}=\boldsymbol{h}_{t}+\boldsymbol{H}_{t} \boldsymbol{z}_{t-1}$

where $\boldsymbol{z}_{t}=\left(\boldsymbol{v}_{t:(t-q+1)}^{2+^{\prime}}, \boldsymbol{v}_{t:(t-q+1)}^{2-^{\prime}}, \mathbf{g}_{t:(t-p+1)}^{\prime}\right)^{\prime}$ and

$\boldsymbol{h}_{t}=\left(\omega_{0}^{\prime} \Upsilon_{t}^{+^{\prime}}, 0_{m(q-1)}^{\prime}, \omega_{0}^{\prime} \Upsilon_{t}^{-^{\prime}}, 0_{(q-1) m}^{\prime}, \omega_{0}^{\prime}, 0_{(p-1) m}^{\prime}\right)^{\prime}$,

where $\boldsymbol{\Upsilon}_{t}^{+}=\operatorname{diag}\left(\boldsymbol{\eta}_{t}^{2+}\right)$ and $\boldsymbol{\Upsilon}_{t}^{-}=\operatorname{diag}\left(\boldsymbol{\eta}_{t}^{2-}\right)$. The matrix $\boldsymbol{H}_{t}$ depends on $\boldsymbol{\theta}_{0}$ and $\boldsymbol{\eta}_{t}$. For example, when $p=q=1$ we have

$\boldsymbol{H}_{t}=\left(\begin{array}{ccc}\boldsymbol{\Upsilon}_{t}^{+} \boldsymbol{A}_{01,+} & \boldsymbol{\Upsilon}_{t}^{+} \boldsymbol{A}_{01,-} & \boldsymbol{\Upsilon}_{t}^{+} \boldsymbol{B}_{01} \\ \boldsymbol{\Upsilon}_{t}^{-} \boldsymbol{A}_{01,+} & \boldsymbol{\Upsilon}_{t}^{-} \boldsymbol{A}_{01,-} & \boldsymbol{\Upsilon}_{t}^{-} \boldsymbol{B}_{01} \\ \boldsymbol{A}_{01,+} & \boldsymbol{A}_{01,-} & \boldsymbol{B}_{01}\end{array}\right)$.

The top Lyapunov exponent of the sequence $\left(\boldsymbol{H}_{t}\right)$ is defined by

$\gamma_{0}=\inf _{t \geq 1} \frac{1}{t} E\left(\log \left\|\boldsymbol{H}_{t} \boldsymbol{H}_{t-1} \ldots \boldsymbol{H}_{1}\right\|\right)$

5 The CholCov of Boudt et al. (2017) is a nonparametric estimator of the quadratic variation of log-prices under asynchronicity and microstructure noise. Like the CHAR model it relies on the Cholesky decomposition and allows a sequential estimation of the covariance matrix. The order of the series also matters in this case. To make optimal use of the data, Boudt et al. (2017) propose an ordering of the series in terms of decreasing liquidity. for any norm $\|\cdot\|$. Denote by $\varrho(\boldsymbol{M})$ the spectral radius of a square matrix $\boldsymbol{M}$, and let $\otimes$ be the Kronecker product of matrices.

Theorem 3.1. Consider the CHAR Model (2.1). A sufficient condition for the existence of a strictly stationary, non anticipative ${ }^{6}$ and ergodic CHAR process $\left(\boldsymbol{\epsilon}_{t}\right)_{t}$ satisfying Eqs. (2.7)-(2.8), or Eqs. (2.7)-(2.9), is $\left.i\right)$ $\gamma_{0}<0$ and (ii) det $\left\{\boldsymbol{I}_{m_{0}}-\sum_{i=1}^{s} \boldsymbol{C}_{0 i} z^{i}\right\} \neq 0$ for all $|z| \leq 1$. Suppose in addition that $\boldsymbol{c}_{0}$ satisfies the Hölder condition iii) $\left\|\boldsymbol{c}_{0}(\boldsymbol{x})-\boldsymbol{c}_{0}(\boldsymbol{y})\right\| \leq$ $K\|\boldsymbol{x}-\boldsymbol{y}\|^{a}$, for some constant $K>0, a \in(0,1]$ and all $\boldsymbol{x}$ and $\boldsymbol{y}$ in $\mathbb{R}^{2 r m}$. Then there exists $s_{0}>0$ such that $E\left\|\boldsymbol{\epsilon}_{1}\right\|^{2 s_{0}}<\infty$ and $E\left\|\Sigma_{1}\right\|^{s_{0}}<\infty$. If $\gamma_{0} \geq 0$ then there exists no stationary solution to (2.7).

Let $m_{1}$ be a positive integer. If

$E\left\|\boldsymbol{\eta}_{1}\right\|^{2 m_{1}}<\infty$ and $\varrho\left(E \boldsymbol{H}_{1}^{\otimes m_{1}}\right)<1$,

then $E\left\|\boldsymbol{v}_{t}\right\|^{2 m_{1}}<\infty$. If, in addition, conditions (ii) and (iii) hold, then $E\left\|\epsilon_{1}\right\|^{2 m_{1}}<\infty$.

For models (2.10)-(2.11), (2.12)-(2.12) and (2.13)-(2.14), the stationarity conditions are more explicit.

Corollary 3.1. There exists a strictly stationary, non anticipative and ergodic process satisfying (2.1) with (2.10)-(2.11), (2.12)-(2.13) or (2.12)-(2.14) when

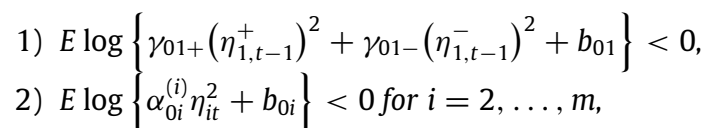

3) $\left|c_{0 i j}\right|<1$ for all $(i, j) \in \boldsymbol{T}_{m}$.

Moreover, the stationary solution satisfies $E\left\|\boldsymbol{\epsilon}_{1}\right\|^{2 s_{0}}<\infty$, $E\left\|\boldsymbol{g}_{1}\right\|^{s_{0}}<\infty, E\left\|\boldsymbol{v}_{1}\right\|^{s_{0}}<\infty, E\left\|\boldsymbol{\beta}_{1}\right\|^{s_{0}}<\infty$ and $E\left\|\boldsymbol{\Sigma}_{1}\right\|^{s_{0}}<\infty$ for some $s_{0}>0$.

\section{One-step and multi-step QMLE}

The parameter $\boldsymbol{\vartheta}_{0}$ can be estimated by the standard Gaussian QMLE. For models with a "triangular structure", such as (2.10)(2.11), a numerically more convenient equation-by-equation estimator (EbEE) can also be used. Proofs are gathered in Section 8 .

\subsection{Full QMLE}

Let $\Theta$ be a compact parameter space which contains $\vartheta_{0}$. For all $\vartheta \in \Theta$, assume that the variance $\boldsymbol{\Sigma}_{t}(\boldsymbol{\vartheta})$ is well-defined, and consider its Cholesky decomposition $\boldsymbol{\Sigma}_{t}(\boldsymbol{\vartheta})=\boldsymbol{L}_{t}(\boldsymbol{\vartheta}) \boldsymbol{G}_{t}(\boldsymbol{\vartheta}) \boldsymbol{L}_{t}^{\prime}(\boldsymbol{\vartheta})$. We also introduce the vector $\boldsymbol{g}_{t}(\boldsymbol{\vartheta})$ of generic element $g_{i t}(\boldsymbol{\vartheta})$ such that $\mathbf{g}_{t}=\mathbf{g}_{t}\left(\boldsymbol{\vartheta}_{0}\right)=\mathbf{g}\left(\boldsymbol{\epsilon}_{t-1}, \boldsymbol{\epsilon}_{t-2}, \ldots ; \boldsymbol{\vartheta}_{0}\right)$, and the vectors $\boldsymbol{\beta}_{t}(\boldsymbol{\vartheta})$ and $\boldsymbol{\ell}_{t}(\boldsymbol{\vartheta})$ such that $\boldsymbol{\beta}_{t}=\boldsymbol{\beta}_{t}\left(\boldsymbol{\vartheta}_{0}\right)=\boldsymbol{\beta}\left(\boldsymbol{\epsilon}_{t-1}, \boldsymbol{\epsilon}_{t-2}, \ldots ; \boldsymbol{\vartheta}_{0}\right)$ and $\boldsymbol{\ell}_{t}=\boldsymbol{\ell}_{t}\left(\boldsymbol{\vartheta}_{0}\right)=\boldsymbol{\ell}\left(\boldsymbol{\epsilon}_{t-1}, \boldsymbol{\epsilon}_{t-2}, \ldots ; \boldsymbol{\vartheta}_{0}\right)$. Given observations $\boldsymbol{\epsilon}_{1}, \ldots, \boldsymbol{\epsilon}_{n}$, and arbitrary fixed initial values $\tilde{\boldsymbol{\epsilon}}_{i}$ for $i \leq 0$, let the statistics

$\widetilde{\Sigma}_{t}(\boldsymbol{\vartheta})=\Sigma\left(\boldsymbol{\epsilon}_{t-1}, \ldots, \boldsymbol{\epsilon}_{1}, \widetilde{\boldsymbol{\epsilon}}_{0}, \widetilde{\boldsymbol{\epsilon}}_{-1}, \ldots ; \boldsymbol{\vartheta}\right)$

and similarly define $\widetilde{\boldsymbol{L}}_{t}(\boldsymbol{\vartheta}), \widetilde{\boldsymbol{G}}_{t}(\boldsymbol{\vartheta})=\operatorname{diag}\left\{\widetilde{\mathbf{g}}_{t}(\boldsymbol{\vartheta})\right\}, \widetilde{\boldsymbol{B}}_{t}(\boldsymbol{\vartheta}), \tilde{\boldsymbol{\ell}}_{t}(\boldsymbol{\vartheta})=$ $\operatorname{vec}^{0} \widetilde{\boldsymbol{L}}_{t}(\boldsymbol{\vartheta})$ and $\widetilde{\boldsymbol{\beta}}_{t}(\boldsymbol{\vartheta})=-\operatorname{vec}^{0} \boldsymbol{B}_{t}(\boldsymbol{\vartheta})$. A QMLE of $\boldsymbol{\vartheta}_{0}$ is defined as any measurable solution $\widehat{\vartheta}_{n}$ of

$\widehat{\boldsymbol{\vartheta}}_{n}=\underset{\boldsymbol{\vartheta} \in \Theta}{\arg \min } \widetilde{O}_{n}(\boldsymbol{\vartheta}), \quad \widetilde{O}_{n}(\boldsymbol{\vartheta})=n^{-1} \sum_{t=1}^{n} \widetilde{q}_{t}(\boldsymbol{\vartheta})$,

where

$$
\begin{aligned}
\widetilde{q}_{t}(\boldsymbol{\vartheta}) & =\boldsymbol{\epsilon}_{t}^{\prime} \widetilde{\boldsymbol{\Sigma}}_{t}^{-1}(\boldsymbol{\vartheta}) \boldsymbol{\epsilon}_{t}+\log \left|\widetilde{\boldsymbol{\Sigma}}_{t}(\boldsymbol{\vartheta})\right| \\
& =\boldsymbol{\epsilon}_{t}^{\prime} \widetilde{\boldsymbol{B}}_{t}^{\prime}(\boldsymbol{\vartheta}) \widetilde{\boldsymbol{G}}_{t}^{-1}(\boldsymbol{\vartheta}) \widetilde{\boldsymbol{B}}_{t}(\boldsymbol{\vartheta}) \boldsymbol{\epsilon}_{t}+\sum_{i=1}^{m} \log \widetilde{g}_{i t}(\boldsymbol{\vartheta}) .
\end{aligned}
$$

$\overline{6}$ i.e $\epsilon_{t}$ measurable with respect to the sigma-field generated by $\left\{\eta_{u}, u \leq t\right\}$. 
It is worth noting that for computing the QMLE, it is not necessary to invert matrices $\left(\widetilde{\boldsymbol{G}}_{t}(\boldsymbol{\vartheta})\right.$ being diagonal). This constitutes an attractive feature of the CHAR models.

\subsubsection{General CHAR model}

We now give high-level assumptions which entail the strong consistency and asymptotic normality (CAN) of the QML estimator for the general CHAR model. These assumptions will be made explicit for the particular model (2.10)-(2.11). In the sequel $\rho$ denotes a generic constant belonging to $[0,1)$, and $K$ denotes a positive constant or a positive random variable measurable with respect to $\left\{\boldsymbol{\epsilon}_{u}, u<0\right\}$ (and thus which does not depend on $n$ ).

A1: $\sup _{\vartheta \in \Theta}\left\|\widetilde{\mathbf{g}}_{t}^{-1}(\boldsymbol{\vartheta})\right\| \leq K, \quad \sup _{\vartheta \in \Theta}\left\|\mathbf{g}_{t}^{-1}(\boldsymbol{\vartheta})\right\| \leq K, \quad$ a.s.

A2: $\sup _{\boldsymbol{\vartheta} \in \Theta}\left\{\left\|\boldsymbol{g}_{t}(\boldsymbol{\vartheta})-\widetilde{\boldsymbol{g}}_{t}(\boldsymbol{\vartheta})\right\|+\left\|\boldsymbol{\beta}_{t}(\boldsymbol{\vartheta})-\widetilde{\boldsymbol{\beta}}_{t}(\boldsymbol{\vartheta})\right\|\right\} \leq K \rho_{t}$ where the random variable $\rho_{t}$ satisfies $\sum_{t=1}^{\infty}\left\{E \rho_{t}^{s_{1}}\right\}^{1 / 3}<\infty$ for all $s_{1} \in\left(0, s_{0}\right]$ and some $s_{0}>0$.

A3: $E\left\{\left\|\boldsymbol{\epsilon}_{t}\right\|^{s_{0}}+\left\|\boldsymbol{g}_{t}\left(\boldsymbol{\vartheta}_{0}\right)\right\|^{s_{0}}+\sup _{\boldsymbol{\vartheta} \in \Theta}\left\|\boldsymbol{\beta}_{t}(\boldsymbol{\vartheta})\right\|^{s_{0}}\right\}<\infty$ for some $s_{0}>0$.

A4: For $\boldsymbol{\vartheta} \in \Theta,\left\{\boldsymbol{g}_{t}(\boldsymbol{\vartheta}), \boldsymbol{\beta}_{t}(\boldsymbol{\vartheta})\right\}=\left\{\boldsymbol{g}_{t}\left(\boldsymbol{\vartheta}_{0}\right), \boldsymbol{\beta}_{t}\left(\boldsymbol{\vartheta}_{0}\right)\right\}$ a.s. implies $\vartheta=\vartheta_{0}$

A5: For any sequence $\boldsymbol{x}_{1}, \boldsymbol{x}_{2}, \ldots$ of vectors of $\mathbb{R}^{m}$, the functions $\boldsymbol{\vartheta} \mapsto \mathbf{g}\left(\boldsymbol{x}_{1}, \boldsymbol{x}_{2}, \ldots ; \boldsymbol{\vartheta}\right)$ from $\Theta$ to $(0,+\infty)^{m}$ and $\boldsymbol{\vartheta} \mapsto$ $\boldsymbol{\beta}\left(\boldsymbol{x}_{1}, \boldsymbol{x}_{2}, \ldots ; \boldsymbol{\vartheta}\right)$ from $\Theta$ to $\mathbb{R}^{m_{0}}$ are continuous on $\Theta$.

A6: $\vartheta_{0}$ belongs to the interior $\Theta$ of $\Theta$.

A7: For any sequence $\boldsymbol{x}_{1}, \boldsymbol{x}_{2}, \ldots$ of vectors of $\mathbb{R}^{m}$, the functions $\boldsymbol{\vartheta} \mapsto \mathbf{g}\left(\boldsymbol{x}_{1}, \boldsymbol{x}_{2}, \ldots ; \boldsymbol{\vartheta}\right)$ and $\boldsymbol{\vartheta} \mapsto \boldsymbol{\beta}\left(\boldsymbol{x}_{1}, \boldsymbol{x}_{2}, \ldots ; \boldsymbol{\vartheta}\right)$ admit continuous second-order derivatives.

A8: For some neighborhood $V\left(\boldsymbol{\vartheta}_{0}\right)$ of $\boldsymbol{\vartheta}_{0}$, there exists $s_{0}>0$ such that

$$
\sup _{\boldsymbol{\vartheta} \in V\left(\boldsymbol{\vartheta}_{0}\right)}\left\{\left\|\frac{\partial \mathbf{g}_{t}(\boldsymbol{\vartheta})}{\partial \boldsymbol{\vartheta}}-\frac{\partial \widetilde{\mathbf{g}}_{t}(\boldsymbol{\vartheta})}{\partial \boldsymbol{\vartheta}}\right\|+\left\|\frac{\partial \boldsymbol{\beta}_{t}(\boldsymbol{\vartheta})}{\partial \boldsymbol{\vartheta}}-\frac{\partial \widetilde{\boldsymbol{\beta}}_{t}(\boldsymbol{\vartheta})}{\partial \boldsymbol{\vartheta}}\right\|\right\} \leq K \rho_{t}
$$

with $\rho_{t}$ as in $\mathbf{A 2}$.

A9: For some neighborhood $V\left(\boldsymbol{\vartheta}_{0}\right)$ of $\boldsymbol{\vartheta}_{0}$, for all $i, j \in\{1, \ldots, m\}$ and $p>0, q>0$ and $r>0$ such that $2 q^{-1}+2 r^{-1}=1$ and $p^{-1}+2 r^{-1}=1$, we have

$$
\begin{aligned}
& E \sup _{\boldsymbol{\vartheta} \in V\left(\boldsymbol{\vartheta}_{0}\right)}\left\|\boldsymbol{\Sigma}_{t}^{-1 / 2^{\prime}}(\boldsymbol{\vartheta}) \frac{\partial^{2} \boldsymbol{\Sigma}_{t}(\boldsymbol{\vartheta})}{\partial \vartheta_{i} \partial \vartheta_{j}} \Sigma_{t}^{-1 / 2}(\boldsymbol{\vartheta})\right\|^{p}<\infty, \\
& E \sup _{\boldsymbol{\vartheta} \in V\left(\boldsymbol{\vartheta}_{0}\right)}\left\|\boldsymbol{\Sigma}_{t}^{-1 / 2^{\prime}}(\boldsymbol{\vartheta}) \frac{\partial \boldsymbol{\Sigma}_{t}(\boldsymbol{\vartheta})}{\partial \vartheta_{i}} \boldsymbol{\Sigma}_{t}^{-1 / 2}(\boldsymbol{\vartheta})\right\|^{q}<\infty, \\
& E \sup _{\boldsymbol{\vartheta} \in V\left(\boldsymbol{\vartheta}_{0}\right)}\left\|\boldsymbol{\Sigma}_{t}^{1 / 2^{\prime}}\left(\boldsymbol{\vartheta}_{0}\right) \boldsymbol{\Sigma}_{t}^{-1 / 2}(\boldsymbol{\vartheta})\right\|^{r}<\infty,
\end{aligned}
$$

where $\vartheta_{i}$ denotes the $i$ th element of the vector $\vartheta$

A10: $E\left\|\boldsymbol{\eta}_{t}\right\|^{4}<\infty$

A11: The matrices $\left\{\partial \Sigma_{t}\left(\vartheta_{0}\right) / \partial \vartheta_{i}, i=1, \ldots, d\right\}$ are linearly independent with nonzero probability.

Theorem 4.1 (CAN of the QMLE in the General CHAR Case). Let $\left(\boldsymbol{\epsilon}_{t}\right)$ be a non anticipative solution to the CHAR model (2.1). Let $\left(\widehat{\vartheta}_{n}\right)$ be a sequence of QML estimators satisfying (4.1). Under A1 -A5 we have

$\widehat{\vartheta}_{n} \rightarrow \vartheta_{0}$, almost surely as $n \rightarrow \infty$.

Under the additional assumptions $\mathbf{A 6}-\mathbf{A 1 0}$, we have the existence of the $d \times d$ matrix

$\boldsymbol{J}=E \boldsymbol{D}_{t}^{\prime}\left\{\boldsymbol{\Sigma}_{t}^{-1}\left(\boldsymbol{\vartheta}_{0}\right) \otimes \boldsymbol{\Sigma}_{t}^{-1}\left(\boldsymbol{\vartheta}_{0}\right)\right\} \boldsymbol{D}_{t}, \quad \boldsymbol{D}_{t}=\frac{\partial \operatorname{vec} \boldsymbol{\Sigma}_{t}\left(\boldsymbol{\vartheta}_{0}\right)}{\partial \boldsymbol{\vartheta}^{\prime}}$,

and of the $d \times d$ matrix $I$ of generic term

$\boldsymbol{I}(i, j)=\operatorname{Tr}\left\{\boldsymbol{K} E \boldsymbol{C}_{i, t} \boldsymbol{C}_{j, t}^{\prime}\right\}$, with $\boldsymbol{K}=\operatorname{Evec}\left(\boldsymbol{I}_{m}-\boldsymbol{\eta}_{t} \boldsymbol{\eta}_{t}^{\prime}\right) \operatorname{vec}^{\prime}\left(\boldsymbol{I}_{m}-\boldsymbol{\eta}_{t} \boldsymbol{\eta}_{t}^{\prime}\right)$ and

$\boldsymbol{C}_{i, t}=\left\{\boldsymbol{\Sigma}_{t}^{-1 / 2}\left(\boldsymbol{\vartheta}_{0}\right) \otimes \Sigma_{t}^{-1 / 2}\left(\boldsymbol{\vartheta}_{0}\right)\right\} \operatorname{vec} \frac{\partial \boldsymbol{\Sigma}_{t}\left(\boldsymbol{\vartheta}_{0}\right)}{\partial \vartheta_{i}}$.

Moreover, under the additional assumption $\mathbf{A 1 1}, \mathbf{J}$ is invertible and $\sqrt{n}\left(\widehat{\vartheta}_{n}-\vartheta_{0}\right) \stackrel{\mathcal{L}}{\rightarrow} \mathcal{N}\left\{0, \boldsymbol{J}^{-1} \boldsymbol{J}^{-1}\right\}$ as $n \rightarrow \infty$.

We also have the Bahadur representation

$\widehat{\boldsymbol{\vartheta}}_{n}-\boldsymbol{\vartheta}_{0}=\boldsymbol{J}^{-1} \frac{1}{n} \sum_{t=1}^{n} \nabla_{t} \operatorname{vec}\left(\boldsymbol{\eta}_{t} \boldsymbol{\eta}_{t}^{\prime}-\boldsymbol{I}_{m}\right)+o_{P}\left(n^{-1 / 2}\right)$,

where $\nabla_{t}=\boldsymbol{D}_{t}^{\prime}\left\{\Sigma_{t}^{-1 / 2^{\prime}}\left(\boldsymbol{\vartheta}_{0}\right) \otimes \Sigma_{t}^{-1 / 2^{\prime}}\left(\vartheta_{0}\right)\right\}$.

\subsubsection{QMLE for a specific parameterization}

Now consider the case where $\left(\boldsymbol{\epsilon}_{t}\right)$ is a stationary and ergodic solution to model (2.10)-(2.11), whose unknown parameter $\boldsymbol{\vartheta}_{0}$ belongs to a compact set $\Theta$ of $\mathbb{R}^{d}$, with $d=m(m+1)(m+5) / 3$. Let $\boldsymbol{\theta}_{0}^{(1)}=\left(\omega_{01}, \gamma_{01+}, \gamma_{01-}, b_{01}\right)^{\prime}$ and, for $i=2, \ldots, m$, let

$\boldsymbol{\theta}_{0}^{(i)}=\left(\omega_{0 i}, \gamma_{0 i_{+}}, \gamma_{0 i_{-}}, \alpha_{0 i}^{(2)}, \ldots, \alpha_{0 i}^{(i)}, b_{0 i}\right)^{\prime}$

be the vector of the $i+3$ parameters involved in $g_{i t}$. Assume that $\boldsymbol{\theta}_{0}^{(i)}$ belongs to a compact set $\Theta_{\boldsymbol{\theta}}^{(i)} \subset(0, \infty) \times[0, \infty)^{i+2}$. For $(i, j) \in \boldsymbol{T}_{m}$, set

$\varphi_{0}^{(i j)}=\left(\varpi_{0 i j}, \varsigma_{0 i j+}, \varsigma_{0 i j-}, \tau_{0 i j}^{(2)}, \ldots, \tau_{0 i j}^{(i)}, c_{0 i j}\right)^{\prime}$,

and $\varphi_{0}^{(i)}=\left(\varphi_{0}^{(i 1)^{\prime}}, \ldots, \varphi_{0}^{(i, i-1)^{\prime}}\right)^{\prime}$. For $i \geq 2$, let $\Theta_{\varphi}^{(i)}$ be the parameter space of $\varphi_{0}^{(i)}$, a compact subspace of $\mathbb{R}^{(i-1)(i+3)}$. The parameter $\varphi_{0}:=$ $\left(\varphi_{0}^{(2)^{\prime}}, \ldots, \varphi_{0}^{(m)^{\prime}}\right)^{\prime}$ belongs to the compact subset $\Theta_{\varphi}=\Theta_{\varphi}^{(2)} \times \cdots \times$ $\Theta_{\varphi}^{(m)}$ of the Euclidean space of dimension $m(m-1)(2 m+11) / 6$. Let $\boldsymbol{\vartheta}_{0}^{(1)}=\boldsymbol{\theta}_{0}^{(1)}$ and $\boldsymbol{\vartheta}_{0}^{(i)}=\left(\boldsymbol{\theta}_{0}^{(i)^{\prime}}, \boldsymbol{\varphi}_{0}^{(i)^{\prime}}\right)^{\prime}$ for $i=2, \ldots, m$. The parameter space $\Theta^{(i)}=\Theta_{\theta}^{(i)} \times \Theta_{\varphi}^{(i)}$ of $\vartheta_{0}^{(i)}$ (with the convention $\left.\Theta^{(1)}=\Theta_{\theta}^{(1)}\right)$ is a compact subset of $\mathbb{R}^{d_{i}}$, with $d_{i}=i(i+3)$. We have $\boldsymbol{\vartheta}_{0}=\left(\boldsymbol{\vartheta}_{0}^{(1)^{\prime}}, \ldots, \boldsymbol{\vartheta}_{0}^{(m)^{\prime}}\right)^{\prime}$. Let $\boldsymbol{\vartheta}=\left(\boldsymbol{\vartheta}^{(1)^{\prime}}, \ldots, \boldsymbol{\vartheta}^{(m)^{\prime}}\right)^{\prime}$ be a generic element of the parameter space $\Theta$ and $\varphi=\left(\varphi^{(2)^{\prime}}, \ldots, \varphi^{(m)^{\prime}}\right)^{\prime}$ a generic element of $\Theta_{\varphi}$. Using (2.3) to compute $v_{k, t-1}$ in (2.11), one can see that $\beta_{i j, t}$ actually depends on

$\varphi_{0}^{(+i)}=\left(\varphi_{0}^{(i)^{\prime}}, \varphi_{0}^{(-i)^{\prime}}\right)^{\prime}, \quad \varphi_{0}^{(-i)}=\left(\varphi_{0}^{(i-1)^{\prime}}, \ldots, \varphi_{0}^{(2)^{\prime}}\right)^{\prime}$,

with the convention $\varphi_{0}^{(+2)}=\varphi_{0}^{(2)}$. We thus consider the function $\beta_{i j, t}\left(\varphi^{(+i)}\right)$ such that $\beta_{i j, t}=\beta_{i j, t}\left(\varphi_{0}^{(+i)}\right)$. Introduce also the notation

$\boldsymbol{\vartheta}_{0}^{(+i)}=\left(\boldsymbol{\vartheta}_{0}^{(i)^{\prime}}, \varphi_{0}^{(-i)^{\prime}}\right)^{\prime}=\left(\boldsymbol{\theta}_{0}^{(i)^{\prime}}, \varphi_{0}^{(+i)^{\prime}}\right)^{\prime}$,

dropping the subscript " 0 " when considering a generic element of a parameter space denoted by $\Theta^{(+i)}$, and using the convention $\vartheta^{(+i)}=\vartheta^{(i)}$ for $i=1$, 2. The QMLE of $\vartheta_{0}$ is the solution to (4.1) where, with some abuse of notation,

$\widetilde{q}_{t}(\boldsymbol{\vartheta})=\sum_{i=1}^{m} \widetilde{q}_{i t}\left(\boldsymbol{\vartheta}^{(+i)}\right)$,

with, for $t=1, \ldots, n$,

$$
\begin{aligned}
& \tilde{q}_{i t}\left(\boldsymbol{\vartheta}^{(+i)}\right)=\frac{\widetilde{v}_{i t}^{2}\left(\boldsymbol{\varphi}^{(+i)}\right)}{\widetilde{g}_{i t}\left(\boldsymbol{\vartheta}^{(+i)}\right)}+\log \widetilde{g}_{i t}\left(\boldsymbol{\vartheta}^{(+i)}\right), \\
& \tilde{g}_{i t}\left(\boldsymbol{\vartheta}^{(+i)}\right)=\omega_{i, t-1}+\sum_{k=2}^{i} \alpha_{i}^{(k)} \widetilde{v}_{k, t-1}^{2}\left(\boldsymbol{\varphi}^{(+k)}\right)+b_{i} \widetilde{g}_{i, t-1}\left(\boldsymbol{\vartheta}^{(+i)}\right) \\
& \tilde{v}_{k t}\left(\boldsymbol{\varphi}^{(+k)}\right)=\epsilon_{k t}-\sum_{j=1}^{k-1} \widetilde{\beta}_{k j, t}\left(\boldsymbol{\varphi}^{(+k)}\right) \epsilon_{j t}
\end{aligned}
$$


$\widetilde{\beta}_{i j, t}\left(\varphi^{(+i)}\right)=\omega_{i j, t-1}+\sum_{k=2}^{i} \tau_{i j}^{(k)} \widetilde{v}_{k, t-1}\left(\varphi^{(+k)}\right)+c_{i j} \widetilde{\beta}_{i j, t-1}\left(\varphi^{(+i)}\right)$,

using the convention $\widetilde{v}_{1 t}^{2}\left(\varphi^{(+i)}\right)=\epsilon_{1 t}^{2}$, the notations $\omega_{i t}=\omega_{i}+$ $\gamma_{i+}\left(\epsilon_{1 t}^{+}\right)^{2}+\gamma_{i-}\left(\epsilon_{1 t}^{-}\right)^{2}$ and $\omega_{i j t}=\varpi_{i j}+\varsigma_{i j+} \epsilon_{1 t}^{+}+\varsigma_{i j-} \epsilon_{1 t}^{-}$, and fixed initial values for $\widetilde{\beta}_{i j, 0}(\cdot), \epsilon_{0}$ and $\widetilde{g}_{i, 0}(\cdot)$.

\subsubsection{Invertibility condition}

Starting with the initial values and using (4.7) for $t=0$ and successively (4.8) and (4.7) for $t=1,2, \ldots$, one can define $\widetilde{\beta}_{i j, t}\left(\varphi^{(+i)}\right)$ for any value of $\varphi^{(+i)}$. One can expect that the initial values be asymptotically negligible, in the sense that, as $t \rightarrow \infty$, $\widetilde{\beta}_{i j, t}\left(\varphi^{(+i)}\right)$ be arbitrarily close to some function $\beta_{i j, t}\left(\varphi^{(+i)}\right)$ measurable with respect to the past observations $\mathcal{F}_{t-1}$. From (4.7), we then consistently approximate $\widetilde{v}_{i t}\left(\varphi^{(+i)}\right)$ by a measurable function $v_{i t}\left(\varphi^{(+i)}\right)$ of $\mathcal{F}_{t-1}$. By imposing $\left|b_{i}\right|<1$ in (4.6), one can also consistently approximate $\widetilde{g}_{i t}\left(\boldsymbol{\vartheta}^{(+i)}\right)$ by some function $g_{i t}\left(\vartheta^{(+i)}\right)$ of $\mathcal{F}_{t-1}$. When these consistent $\mathcal{F}_{t-1}$-approximations hold for all values of $i$ and $j$, the model (2.10)-(2.11) is said to be invertible. For prediction or estimation purposes, it is important that the model possesses this property. Indeed, if the invertibility does not hold, the approximate volatility $\widetilde{\boldsymbol{\Sigma}}_{t}(\boldsymbol{\vartheta})$ is likely to depend strongly on the initial values, even for large $t$, and thus the model would provide unstable predictions that depend much on the initial values. The noninvertibility would also entail estimation inconsistency, because the objective functions $\widetilde{O}_{n}^{(i)}\left(\vartheta^{(+i)}\right)$ could asymptotically depend on the initial values. Problems of invertibility have been documented in Wintenberger (2013) for the EGARCH model of Nelson (1991) and more recently Blasques et al. (2016) for the Beta-t GARCH of Harvey (2013). In both models, the conditional variance (or its $\log$ ) depends in a very nonlinear way on past innovations.

We now give conditions for the uniform invertibility of Model (2.10)-(2.11).

For all $\varphi \in \Theta_{\varphi}$, let

$$
\begin{aligned}
\widetilde{\boldsymbol{\beta}}_{t}(\boldsymbol{\varphi}) & =\left(\widetilde{\beta}_{21, t}\left(\boldsymbol{\varphi}^{(2)}\right), \widetilde{\beta}_{31, t}\left(\boldsymbol{\varphi}^{(+3)}\right) \ldots, \widetilde{\beta}_{m, m-1, t}\left(\boldsymbol{\varphi}^{(+m)}\right)\right)^{\prime} \\
& =-\operatorname{vech}^{0} \widetilde{\boldsymbol{B}}_{t}(\boldsymbol{\varphi}),
\end{aligned}
$$

where $\widetilde{\boldsymbol{B}}_{t}(\boldsymbol{\varphi})$ is a lower unitriangular matrix. In vector form, the Eqs. (4.7)-(4.8) write

$$
\widetilde{\boldsymbol{\beta}}_{t}(\boldsymbol{\varphi})=\boldsymbol{w}_{t-1}+\boldsymbol{T} \widetilde{\boldsymbol{B}}_{t-1}(\boldsymbol{\varphi}) \boldsymbol{\epsilon}_{t-1}+\boldsymbol{C} \widetilde{\boldsymbol{\beta}}_{t-1}(\boldsymbol{\varphi}), \quad t \geq 1,
$$

with a fixed initial values for $\widetilde{\boldsymbol{\beta}}_{0}(\boldsymbol{\varphi})$. If the $\ell$ th element of $\boldsymbol{\beta}_{t}$ contains $\beta_{i j, t}$, then the $\ell$ th element of the random vector $\boldsymbol{w}_{t}$ is $\omega_{i j t}$, row $\ell$ of $\boldsymbol{T}$ is $\left(0, \tau_{i j}^{(2)}, \ldots, \tau_{i j}^{(i)}, \mathbf{0}_{m-i}^{\prime}\right)$, and $\mathbf{C}$ is a diagonal matrix with $c_{i j}$ as $\ell$ th diagonal element. Let $\mathbf{D}_{m}^{0}$ be the matrix with elements 0 or 1 such that vec $\boldsymbol{A}=\mathbf{D}_{m}^{0} \operatorname{vech}^{0} \boldsymbol{A}$ for any strictly lower triangular ${ }^{7} m \times m$ matrix $\boldsymbol{A}$. Noting that vec $\widetilde{\boldsymbol{B}}_{t}(\boldsymbol{\varphi})=-\mathbf{D}_{m}^{0} \widetilde{\boldsymbol{\beta}}_{t}(\boldsymbol{\varphi})+\operatorname{vec} \boldsymbol{I}_{m}$, we obtain

$$
\boldsymbol{T}_{\boldsymbol{B}}(\boldsymbol{\varphi}) \boldsymbol{\epsilon}_{t}=\boldsymbol{T} \boldsymbol{\epsilon}_{t}-\left(\boldsymbol{\epsilon}_{t}^{\prime} \otimes \boldsymbol{T}\right) \mathbf{D}_{m}^{0} \widetilde{\boldsymbol{\beta}}_{t}(\boldsymbol{\varphi})
$$

and

$$
\begin{aligned}
\widetilde{\boldsymbol{\beta}}_{t}(\boldsymbol{\varphi})= & \boldsymbol{w}_{t-1}^{*}+\sum_{i=1}^{t-1}\left(\prod_{j=1}^{i} \boldsymbol{s}_{t-j}\right) \boldsymbol{w}_{t-i-1}^{*} \\
& +\left(\prod_{i=1}^{t-1} \boldsymbol{S}_{t-i}\right)\left(\boldsymbol{w}_{0}^{*}+\widetilde{\boldsymbol{\beta}}_{0}(\boldsymbol{\varphi})\right),
\end{aligned}
$$

\footnotetext{
7 By strictly lower triangular we mean a lower triangular matrix whose main diagonal consists of zeros.
}

with $\boldsymbol{w}_{t}^{*}=\boldsymbol{w}_{t}+\boldsymbol{T} \boldsymbol{\epsilon}_{t}$ and $\boldsymbol{S}_{t}=\boldsymbol{S}_{t}(\boldsymbol{\varphi})=\boldsymbol{C}-\left(\boldsymbol{\epsilon}_{t}^{\prime} \otimes \boldsymbol{T}\right) \mathbf{D}_{m}^{0}$. By the Cauchy rule, under the conditions

$$
\begin{aligned}
& E \log ^{+} \sup _{\varphi \in \Theta_{\varphi}}\left\|\boldsymbol{w}_{1}+\boldsymbol{T} \boldsymbol{\epsilon}_{1}\right\|<\infty, \\
& \gamma_{S}:=\limsup _{n \rightarrow \infty} \frac{1}{n} \log \sup _{\varphi \in \Theta_{\varphi}}\left\|\prod_{i=1}^{n} \boldsymbol{s}_{t-i}\right\|<0,
\end{aligned}
$$

where $\|\cdot\|$ denotes any multiplicative norm, the series

$$
\boldsymbol{\beta}_{t}(\boldsymbol{\varphi})=\boldsymbol{w}_{t-1}^{*}+\sum_{i=1}^{\infty}\left(\prod_{j=1}^{i} \boldsymbol{s}_{t-j}\right) \boldsymbol{w}_{t-i-1}^{*}
$$

converges absolutely (and uniformly) with probability one. Moreover,

$$
\begin{aligned}
\sup _{\boldsymbol{\varphi} \in \Theta_{\varphi}}\left\|\boldsymbol{\beta}_{t}(\boldsymbol{\varphi})-\widetilde{\boldsymbol{\beta}}_{t}(\boldsymbol{\varphi})\right\| & =\sup _{\boldsymbol{\varphi} \in \Theta \varphi}\left\|\left(\prod_{i=1}^{t-2} \boldsymbol{S}_{t-i}\right)\left\{\boldsymbol{\beta}_{1}(\boldsymbol{\varphi})-\widetilde{\boldsymbol{\beta}}_{1}(\boldsymbol{\varphi})\right\}\right\| \\
& \leq K \rho^{t}
\end{aligned}
$$

where $K$ is a positive random variable measurable with respect to $\left\{\boldsymbol{\epsilon}_{u}, u \leq 0\right\}$ and $0 \leq \exp \left\{\gamma_{s}(\boldsymbol{\varphi})\right\}<\rho<1$ (see Bougerol and Picard, $1992 a$, b for conditions under which (4.11) is not only sufficient but also necessary for the existence of (4.12)).

Note that for any $s_{0}>0$, there exists $K>0$ such that $\log ^{+}\left\|\boldsymbol{w}_{1}\right\| \leq K+\left\|\boldsymbol{w}_{1}+\boldsymbol{T} \boldsymbol{\epsilon}_{1}\right\|^{s_{0}}$. Therefore $E \log ^{+}\left\|\boldsymbol{w}_{1}+\boldsymbol{T} \boldsymbol{\epsilon}_{1}\right\|<\infty$ if $E\left\|\boldsymbol{\epsilon}_{1}\right\|^{s_{0}}<\infty$ for some $s_{0}>0$, regardless of the value of $\boldsymbol{\varphi}$. Consequently, in view of Corollary 3.1, Condition (4.10) does not constrain the compact parameter space $\Theta_{\varphi}$. By contrast, (4.11) entails non explicit constraints on $\varphi$. By Jensen's inequality, the condition $\gamma_{S}<0$ is satisfied if $\operatorname{Esup}_{\varphi \in \Theta_{\varphi}}\left\|\boldsymbol{S}_{1}\right\|<1$ for some multiplicative norm $\|\cdot\|$. Take the spectral norm, defined by $\|\boldsymbol{A}\|_{2}=$ $\sqrt{\varrho\left(\boldsymbol{A}^{*} \boldsymbol{A}\right)}$, with $\varrho$ the spectral radius and other standard notations. Noting that $\left\|\mathbf{D}_{m}^{0}\right\|_{2}=\sqrt{2}$ and $\left\|\boldsymbol{\epsilon}_{t}^{\prime} \otimes \boldsymbol{T}\right\|_{2}=\sqrt{\varrho\left(\boldsymbol{\epsilon}_{t} \boldsymbol{\epsilon}_{t}^{\prime} \otimes \boldsymbol{T}^{\prime} \boldsymbol{T}\right)}=$ $\sqrt{\boldsymbol{\epsilon}_{t}^{\prime} \boldsymbol{\epsilon}_{t}}\|\boldsymbol{T}\|_{2}$, and using the compactness of $\Theta_{\varphi}$, we obtain that the uniform invertibility holds if

$$
\max _{i, j}\left|c_{0 i j}\right|+\sqrt{2}\|\boldsymbol{T}\|_{2} E \sqrt{\boldsymbol{\epsilon}_{1}^{\prime} \boldsymbol{\epsilon}_{1}}<1, \quad \forall \varphi \in \Theta_{\varphi} .
$$

Note that, although slightly more explicit, Condition (4.14) is more restrictive than Condition (4.11). In particular, (4.14) requires a finite second order moment for $\left\|\boldsymbol{\epsilon}_{t}\right\|$ when $\boldsymbol{T} \neq \mathbf{0}$. Note also that if the term $\sum_{k=2}^{i} \tau_{0 i j}^{(k)} v_{k, t-1}$ does not appear in (2.11), then the model is invertible, i.e., (4.11) holds, if $\max _{i, j}\left|c_{0 i j}\right|<1$ for all $\varphi \in \Theta_{\varphi}$.

\subsubsection{Asymptotic property of the QMLE}

Let us define the following empirical information matrices:

$\boldsymbol{J}_{n}=\frac{\partial^{2} \widetilde{O}_{n}\left(\widehat{\boldsymbol{\vartheta}}_{n}\right)}{\partial \boldsymbol{\vartheta} \partial \boldsymbol{\vartheta}^{\prime}} \quad$ and $\quad \boldsymbol{I}_{n}=\frac{1}{n} \sum_{t=1}^{n} \frac{\partial \widetilde{q}_{t}\left(\widehat{\boldsymbol{\vartheta}}_{n}\right)}{\partial \boldsymbol{\vartheta}} \frac{\partial \widetilde{q}_{t}\left(\widehat{\vartheta}_{n}\right)}{\partial \boldsymbol{\vartheta}^{\prime}}$.

We need the following assumptions.

B1: Conditions (1), (2) and (3) of Corollary 3.1 are satisfied.

B2: For $i=2, \ldots, m$, the distribution of $\eta_{i t}^{2}$ conditionally on $\left\{\eta_{j t}, j \neq i\right\}$ is non-degenerate. The support of $\eta_{1 t}$ contains at least two positive points and two negative points.

B3: For $(i, j) \in \mathfrak{T}_{m}$ and all $\boldsymbol{\vartheta}^{(i)} \in \Theta^{(i)}$ we have $\boldsymbol{\theta}^{(i)} \geq 0$ componentwise, $\omega_{0 i}>0,\left|b_{0 i}\right|<1$ and $\left|c_{0 i j}\right|<1$.

B4: For $i=1, \ldots, m$, we have $\left(\gamma_{0 i_{+}}, \gamma_{0 i_{-}}, \alpha_{0 i}^{(2)}, \ldots, \alpha_{0 i}^{(i)}\right) \neq 0$ and $\left(\varsigma_{0 i j+}, \varsigma_{0 i j-}, \tau_{0 i j}^{(2)}, \ldots, \tau_{0 i j}^{(i)}\right) \neq 0$.

B5: The uniform invertibility condition (4.11) holds true.

B6: There exist three pairs of Hölder conjugate numbers, $\left(p_{i}, q_{i}\right)$ with $p_{i}^{-1}+q_{i}^{-1}=1$ for $i \in\{1,2,3\}$, such that $E\left\|\epsilon_{t}\right\|^{4 p_{1} q_{2}}$ 
$<\infty, E\left\|\epsilon_{t}\right\|^{2 q_{1} q_{3}}<\infty$, and there exists a neighborhood $V\left(\varphi_{0}\right)$ of $\varphi_{0}$ such that

$$
\begin{aligned}
& E \sup _{\boldsymbol{\varphi} \in V\left(\varphi_{0}\right)}\left\|\boldsymbol{\beta}_{t}(\boldsymbol{\varphi})\right\|^{4 p_{1} p_{2}}<\infty, \\
& E \sup _{\boldsymbol{\varphi} \in V\left(\varphi_{0}\right)}\left\|\frac{\partial \boldsymbol{\beta}_{t}(\boldsymbol{\varphi})}{\partial \boldsymbol{\varphi}^{\prime}}\right\|^{2 q_{1} p_{3}}<\infty,
\end{aligned}
$$

where $\boldsymbol{\beta}_{t}(\boldsymbol{\varphi})$ is defined by (4.12).

In the case where the conditional betas are constant, (4.15) holds true for any value of the Hölder conjugates. Therefore one can choose $q_{1}=3$ and $q_{2}, q_{3}$ arbitrarily close to 1 , which shows that, in this case, $\mathbf{B} \mathbf{6}$ is satisfied when $\left\|\boldsymbol{\epsilon}_{t}\right\|$ admits a moment larger than 6.

As shown by Corollary 3.1, B1 ensures the existence of a strictly stationary solution to (2.10)-(2.11). Assumption B2 is an identifiability assumption. Assumption $\mathbf{B} 3$ guarantees the existence and positivity of $\widetilde{g}_{i t}\left(\boldsymbol{\vartheta}^{(i)}\right)$, as well as the existence of $\widetilde{\beta}_{i j, t}\left(\boldsymbol{\vartheta}^{(i)}\right)$. The first part of $\mathbf{B} 4$ avoids the well-known identifiability issue $g_{i t}=$ $\omega_{0 i} /\left(1-b_{0 i}\right)=\omega_{0 i}-b_{0 i} g_{i, t-1}$ that arises when all the coefficients $\gamma_{0 i+}, \gamma_{0 i-}, \alpha_{0 i}^{(2)}, \ldots, \alpha_{0 i}^{(i)}$ are equal to zero. The second part is an analogous identifiability condition for $\beta_{i j, t}$. Assumption B5 ensures that the initial values are asymptotically negligible.

The moment conditions in $\mathbf{B} \mathbf{6}$ are required for the asymptotic normality (see the example given after the next theorem). It must be acknowledged that they are restrictive and not explicit. Indeed, Theorem 3.1 provides more explicit conditions for even-order moments only. Even for the simplest univariate ARCH processes $\epsilon_{t}$, the precise conditions for the existence of $E\left|\epsilon_{t}\right|^{p_{1}}$ are unknown when $p_{1}$ is not an even integer. To our knowledge, the only asymptotic theory available for multivariate GARCH models with dynamic conditional correlation has been provided by Comte and Lieberman (2003) and concerns the BEKK model. These authors assume, in particular, a moment of order two for the consistency and of order eight for the asymptotic normality. Actually, Avarucci et al. (2013) give evidence that moment conditions are necessary for the CAN of the QMLE in the case of the simplest VEC-GARCH model, which contrasts with the univariate GARCH models for which the CAN can be established without moment conditions on the observed process.

Theorem 4.2 (CAN of the QMLE). Consider the CHAR model (2.1) satisfying (2.10)-(2.11). Under B1 -B5, the QMLE $\widehat{\vartheta}_{n}$ defined by (4.1) and (4.4)-(4.8) satisfies

$\widehat{\vartheta}_{n} \rightarrow \vartheta_{0}$, almost surely as $n \rightarrow \infty$.

Under the additional assumptions $\mathbf{A 6}, \boldsymbol{A 1 0}$ and $\mathbf{B 6}$, the matrices $\boldsymbol{I}_{n}$ and $\boldsymbol{J}_{n}$ converge almost surely to positive-definite matrices $\mathbf{I}$ and $\boldsymbol{J}$ as $n \rightarrow \infty$. Moreover,

$\sqrt{n}\left(\widehat{\vartheta}_{n}-\vartheta_{0}\right) \stackrel{\mathcal{L}}{\rightarrow} \mathcal{N}(0, \Omega), \quad \Omega=\boldsymbol{J}^{-1} \boldsymbol{J}^{-1}$

In order to emphasize the need of the moment conditions involved in B6, let us consider an elementary bivariate example. Assume $g_{1 t}=\omega_{01}+\gamma_{01+}\left(\epsilon_{1, t-1}^{+}\right)^{2}+\gamma_{01-}\left(\epsilon_{1, t-1}^{+}\right)^{2}+b_{01} g_{1, t-1}, g_{2 t}=$ $\omega_{02}$ and $\beta_{21, t}=\varpi_{0}+\varsigma_{0+} \epsilon_{1, t-1}^{+}+\varsigma_{0-} \epsilon_{1, t-1}^{-}$. There are 8 unknown parameters and, in view of (8.13) in the proof of Theorem 4.2, we have

$I(8,8)=\operatorname{Var}\left(2 \eta_{2 t} \frac{1}{\sqrt{g_{2 t}}} \frac{\partial v_{2 t}}{\partial \varsigma_{-}}\right)=\frac{4}{\omega_{02}} E \epsilon_{1, t}^{2}\left(\epsilon_{1, t-1}^{-}\right)^{2}$.

Therefore, even in this simplistic example, at least moments of order 4 are required.

\subsection{Multi-step QMLE}

Recall that (2.10)-(2.11) defines a triangular system in which the dynamics of the $i$ th row depends only on the dynamics of the previous rows. Therefore, instead of estimating simultaneously the $d$ components of $\boldsymbol{\vartheta}_{0}$, one can estimate the parameters equationby-equation (EbE) (see e.g., Francq and Zakoïan, 2016 for an EbE estimator in another class of multivariate GARCH models).

\subsubsection{Definition of the estimator}

For simplicity, the EbE estimator (EbEE) of $\boldsymbol{\vartheta}_{0}$ is described in the case of Model (2.10)-(2.11), but the estimator can be readily extended to other models.

In a first step we estimate $\vartheta_{0}^{(1)}$ by

$\widehat{\vartheta}_{n}^{(1)}=\underset{\vartheta^{(1)} \in \Theta^{(1)}}{\arg \min } \widetilde{O}_{n}^{(1)}\left(\vartheta^{(1)}\right), \quad \widetilde{O}_{n}^{(1)}\left(\vartheta^{(1)}\right)=\frac{1}{n} \sum_{t=1}^{n} \widetilde{q}_{1 t}\left(\boldsymbol{\vartheta}^{(1)}\right)$,

where, according to (4.5), $\widetilde{q}_{1 t}\left(\vartheta^{(1)}\right)$ is defined by

$\widetilde{q}_{1 t}\left(\boldsymbol{\vartheta}^{(1)}\right)=\frac{\epsilon_{1 t}^{2}}{\widetilde{g}_{1 t}\left(\boldsymbol{\vartheta}^{(1)}\right)}+\log \widetilde{g}_{1 t}\left(\boldsymbol{\vartheta}^{(1)}\right)$,

$\tilde{g}_{1 t}\left(\boldsymbol{\vartheta}^{(1)}\right)=\omega_{1, t-1}+b_{1} \widetilde{g}_{1, t-1}\left(\boldsymbol{\vartheta}^{(1)}\right)$.

The estimation of $\vartheta_{0}^{(2)}$ can be done independently or in parallel to $\vartheta_{0}^{(1)}$ by using

$\widehat{\vartheta}_{n}^{(2)}=\underset{\vartheta^{(2)} \in \Theta^{(2)}}{\arg \min } \widetilde{O}_{n}^{(2)}\left(\boldsymbol{\vartheta}^{(2)}\right), \quad \widetilde{O}_{n}^{(2)}\left(\boldsymbol{\vartheta}^{(2)}\right)=\frac{1}{n} \sum_{t=1}^{n} \tilde{q}_{2 t}\left(\boldsymbol{\vartheta}^{(2)}\right)$,

where, following again (4.5),

$$
\begin{aligned}
\tilde{q}_{2 t}\left(\boldsymbol{\vartheta}^{(2)}\right) & =\frac{\widetilde{v}_{2 t}^{2}\left(\boldsymbol{\varphi}^{(2)}\right)}{\widetilde{g}_{2 t}\left(\boldsymbol{\vartheta}^{(2)}\right)}+\log \widetilde{g}_{2 t}\left(\boldsymbol{\vartheta}^{(2)}\right), \\
\widetilde{g}_{2 t}\left(\boldsymbol{\vartheta}^{(2)}\right) & =\omega_{2, t-1}+\alpha_{2}^{(2)} \widetilde{v}_{2, t-1}^{2}\left(\boldsymbol{\varphi}^{(2)}\right)+b_{2} \widetilde{g}_{2, t-1}\left(\boldsymbol{\varphi}^{(2)}\right), \\
\widetilde{v}_{2 t}\left(\boldsymbol{\varphi}^{(2)}\right) & =\epsilon_{2 t}-\widetilde{\beta}_{21, t}\left(\boldsymbol{\varphi}^{(2)}\right) \epsilon_{1 t}, \\
\widetilde{\beta}_{21, t}\left(\boldsymbol{\varphi}^{(2)}\right) & =\omega_{21, t-1}+\tau_{21}^{(2)} \widetilde{v}_{2, t-1}\left(\varphi^{(2)}\right)+c_{21} \widetilde{\beta}_{21, t-1}\left(\varphi^{(2)}\right) .
\end{aligned}
$$

For $i=3, \ldots, m$, the estimation of $\vartheta_{0}^{(i)}$ depends on the estimates $\widehat{\boldsymbol{\varphi}}_{n}^{(-i)}=\left(\widehat{\boldsymbol{\varphi}}_{n}^{(i-1)^{\prime}}, \ldots, \widehat{\boldsymbol{\varphi}}_{n}^{(2)^{\prime}}\right)^{\prime}$ obtained in previous steps (i.e., $j<i$ ). We then estimate $\vartheta_{0}^{(i)}$, for $i=3, \ldots, m$, by

$\widehat{\vartheta}_{n}^{(i)}=\arg \min _{\vartheta^{(i)} \in \Theta^{(i)}} \widetilde{O}_{n}^{(i)}\left(\begin{array}{c}\boldsymbol{\vartheta}^{(i)} \\ \widehat{\boldsymbol{\varphi}}_{n}^{(-i)}\end{array}\right)$,

$\widetilde{O}_{n}^{(i)}\left(\boldsymbol{\vartheta}^{(+i)}\right)=\frac{1}{n} \sum_{t=1}^{n} \widetilde{q}_{i t}\left(\vartheta^{(+i)}\right)$,

where $\widetilde{q}_{i t}$ is defined in (4.5).

\subsubsection{Asymptotic behavior of the EbEE}

Let us define the following empirical information matrices:

$\boldsymbol{J}_{n}^{(i)}=\frac{\partial^{2} \widetilde{\boldsymbol{O}}_{n}^{(i)}\left(\widehat{\boldsymbol{\vartheta}}_{n}^{(+i)}\right)}{\partial \boldsymbol{\vartheta}^{(i)} \partial \boldsymbol{\vartheta}^{(i)^{\prime}}}, \quad \boldsymbol{I}_{n}^{(i)}=\frac{1}{n} \sum_{t=1}^{n} \frac{\partial \widetilde{q}_{i t}\left(\widehat{\boldsymbol{\vartheta}}_{n}^{(+i)}\right)}{\partial \boldsymbol{\vartheta}^{(i)}} \frac{\partial \widetilde{q}_{i t}\left(\widehat{\boldsymbol{\vartheta}}_{n}^{(+i)}\right)}{\partial \boldsymbol{\vartheta}^{(i)^{\prime}}}$,

$\boldsymbol{K}_{n}^{(i)}=\frac{\partial^{2} \widetilde{O}_{n}^{(i)}\left(\widehat{\boldsymbol{\vartheta}}_{n}^{(+i)}\right)}{\partial \boldsymbol{\vartheta}^{(i)} \partial \varphi^{(-i)^{\prime}}}$

with the notation $\widehat{\vartheta}_{n}^{(+i)}=\widehat{\boldsymbol{\vartheta}}_{n}^{(i)}$ for $i=1,2$ and $\widehat{\vartheta}_{n}^{(+i)}=\left(\widehat{\boldsymbol{\vartheta}}_{n}^{(i)^{\prime}}, \widehat{\boldsymbol{\varphi}}_{n}^{(-i)^{\prime}}\right)^{\prime}$ for $i=3, \ldots, m$. By definition, the components of $\eta_{t}$ are uncorrelated. The form of the asymptotic variance of the EbEE can be simplified under the assumption that the components of $\eta_{t}$ are independent, or more generally under the following assumption. 
B7: The components of $\boldsymbol{\eta}_{t}$ are such that $E \eta_{i t} \eta_{j t}^{2}=0$ and $E \eta_{i t}^{2} \eta_{j t}^{2}=$ 1 when $i \neq j$.

Theorem 4.3 (CAN of the EbEE). Consider Model (2.1), satisfying (2.10)-(2.11). Under B1 -B5, the EbEE $\widehat{\vartheta}_{n}^{E b E}=\left(\widehat{\boldsymbol{\vartheta}}_{n}^{(1)^{\prime}}, \ldots, \widehat{\boldsymbol{\vartheta}}_{n}^{(m)^{\prime}}\right)^{\prime}$ defined by (4.17), (4.18) and (4.19) satisfies

$\widehat{\vartheta}_{n}^{E b E} \rightarrow \vartheta_{0}$, almost surely as $n \rightarrow \infty$.

Under the additional assumptions $\mathbf{A 6 ,} \boldsymbol{A 1 0}$ and $\mathbf{B 6}$, for $i=1, \ldots, m$, the matrices $\boldsymbol{J}_{n}^{(i)}, \boldsymbol{I}_{n}^{(i)}$ and $\boldsymbol{K}_{n}^{(i)}$ converge almost surely, respectively, to a positive-definite matrix $\boldsymbol{J}^{(i)}$, to a positive semidefinite matrix $\boldsymbol{I}^{(i)}$, and to a matrix $\boldsymbol{K}^{(i)}$, as $n \rightarrow \infty$. Moreover,

$\sqrt{n}\left(\widehat{\vartheta}_{n}^{(i)}-\vartheta_{0}^{(i)}\right) \stackrel{\mathcal{L}}{\rightarrow} \mathcal{N}\left\{0, \Sigma^{(i)}:=\left(\boldsymbol{J}^{(i)}\right)^{-1} \boldsymbol{I}^{(i)}\left(\boldsymbol{J}^{(i)}\right)^{-1}\right\}$

for $i=1$ and $i=2$. According to (4.3), denote by $\Sigma_{\varphi^{-}}^{(+i)}$ (or by $\Sigma_{\varphi^{+}}^{(+(i-1))}$ ) the bottom-right sub-matrix of $\Sigma^{(+i)}$ (or of $\Sigma^{(+(i-1))}$ ) corresponding to the asymptotic variance of $\widehat{\varphi}_{n}^{(-i)}$ (which is equal to $\left.\widehat{\varphi}_{n}^{(+(i-1))}\right)$. Using this notation and the convention $\Sigma^{(+2)}=\Sigma^{(2)}$, for $i=3, \ldots, m$, we have

$\sqrt{n}\left(\widehat{\vartheta}_{n}^{(+i)}-\vartheta_{0}^{(+i)}\right) \stackrel{\mathcal{L}}{\rightarrow} \mathcal{N}\left(0, \Sigma^{(+i)}\right)$

with, under B7,

$\Sigma^{(+i)}=\left(\begin{array}{cc}\Sigma_{\vartheta}^{(i)} & -\left(\boldsymbol{J}^{(i)}\right)^{-1} \boldsymbol{K}^{(i)} \Sigma_{\varphi^{+}}^{(+(i-1))} \\ -\Sigma_{\varphi^{+}}^{(+(i-1))} \boldsymbol{K}^{(i)^{\prime}}\left(\boldsymbol{J}^{(i)}\right)^{-1} & \Sigma_{\varphi^{+}}^{(+(i-1))}\end{array}\right)$

and $\Sigma_{\vartheta}^{(i)}=\left(\boldsymbol{J}^{(i)}\right)^{-1}\left\{\boldsymbol{I}^{(i)}+\boldsymbol{K}^{(i)} \Sigma_{\varphi^{+}}^{(+(i-1))} \boldsymbol{K}^{(i)^{\prime}}\right\}\left(\boldsymbol{J}^{(i)}\right)^{-1}$.

\subsection{Comparison between the full QMLE and the EbEE}

Consider the CHAR model (2.1) satisfying (2.10)-(2.11). If $m=$ 2, the one-step full QMLE and the two-step EbEE are exactly the same because

$\widetilde{Q}_{n}(\boldsymbol{\vartheta})=\widetilde{Q}_{n}^{(1)}\left(\boldsymbol{\vartheta}^{(1)}\right)+\widetilde{Q}_{n}^{(2)}\left(\boldsymbol{\vartheta}^{(2)}\right)$

in this case. For $m \geq 3$, the two estimators are generally different. In order to compare their asymptotic accuracies, we consider a simplistic static model with $g_{i t}=1$ for $i \in\{1,2,3\}, \ell_{21, t}=$ $\ell_{21}, \ell_{32, t}=\ell_{32}$ and $\ell_{31, t}=0$ (or equivalently $\beta_{21, t}=\beta_{21}$, $\beta_{32, t}=\beta_{32}$ and $\beta_{31, t}=-\beta_{21} \beta_{32}$ ). The unknown parameter is thus $\vartheta=\left(\beta_{21}, \beta_{32}\right)$. The full QMLE is

$\widehat{\boldsymbol{\vartheta}}_{n}=\underset{\boldsymbol{\vartheta} \in \Theta}{\arg \min } \sum_{t=1}^{n} q_{t}(\boldsymbol{\vartheta})$,

where $q_{t}(\boldsymbol{\vartheta})=\left(\epsilon_{2 t}-\beta_{21} \epsilon_{1 t}\right)^{2}+\left(\epsilon_{3 t}-\beta_{32} \epsilon_{2 t}+\beta_{21} \beta_{32} \epsilon_{1 t}\right)^{2}$ and $\Theta$ is a compact parameter space such that $\vartheta_{0} \in \stackrel{\Theta}{\Theta}$. We have

$$
\frac{\partial q_{t}(\boldsymbol{\vartheta})}{\partial \boldsymbol{\vartheta}}=\left(\begin{array}{c}
-2 \epsilon_{1 t} v_{2 t}(\boldsymbol{\vartheta})+2 \beta_{32} \epsilon_{1 t} v_{3 t}(\boldsymbol{\vartheta}) \\
-2 v_{2 t}(\boldsymbol{\vartheta}) v_{3 t}(\boldsymbol{\vartheta})
\end{array}\right)
$$

and

$$
\begin{aligned}
& \frac{\partial^{2} q_{t}(\boldsymbol{\vartheta})}{\partial \boldsymbol{\vartheta} \partial \boldsymbol{\vartheta}^{\prime}} \\
& \quad=\left(\begin{array}{cc}
2 \epsilon_{1 t}^{2}+2 \beta_{32}^{2} \epsilon_{1 t}^{2} & 2 \epsilon_{1 t} v_{3 t}(\boldsymbol{\vartheta})-2 \beta_{32} \epsilon_{1 t} v_{2 t}(\boldsymbol{\vartheta}) \\
2 \epsilon_{1 t} v_{3 t}(\boldsymbol{\vartheta})-2 \beta_{32} \epsilon_{1 t} v_{2 t}(\boldsymbol{\vartheta}) & 2 v_{2 t}^{2}(\boldsymbol{\vartheta})
\end{array}\right)
\end{aligned}
$$

with

$v_{2 t}(\boldsymbol{\vartheta})=\epsilon_{2 t}-\beta_{21} \epsilon_{1 t}, \quad v_{3 t}(\boldsymbol{\vartheta})=\epsilon_{3 t}-\beta_{32} \epsilon_{2 t}+\beta_{21} \beta_{32} \epsilon_{1 t}$.

Assume for instance that the variable $\eta_{2 t}$ is independent of the vector $\left(\eta_{1 t}, \eta_{3 t}\right)^{\prime}$ and that this vector is distributed as the product $\eta \boldsymbol{u}$, where the random variable $\eta$ and the vector $\boldsymbol{u}$ are independent, e.g., $\boldsymbol{u} \sim \mathcal{N}\left(\mathbf{0}, \boldsymbol{I}_{2}\right)$ and $E \eta^{2}=1$. In view of Theorem 4.1, and noting that $v_{i t}\left(\boldsymbol{\vartheta}_{0}\right)=\eta_{i t}$, we then have

$$
\begin{aligned}
\sqrt{n}\left(\widehat{\vartheta}_{n}-\vartheta_{0}\right) & \stackrel{o_{P}(1)}{=}\left\{E \frac{\partial^{2} q_{t}\left(\vartheta_{0}\right)}{\partial \vartheta \partial \vartheta^{\prime}}\right\}^{-1} \frac{1}{\sqrt{n}} \sum_{t=1}^{n} \frac{\partial q_{t}\left(\vartheta_{0}\right)}{\partial \vartheta} \\
& \stackrel{\mathcal{L}}{\rightarrow} \mathcal{N}\left\{0, \Omega:=\left(\begin{array}{cc}
\frac{1+\beta_{32}^{2} E \eta^{4}}{\left(1+\beta_{32}^{2}\right)^{2}} & 0 \\
0 & E \eta^{4}
\end{array}\right)\right\} .
\end{aligned}
$$

The EbEE of the parameter $\beta_{21}$ is defined by

$$
\widehat{\beta}_{21}=\underset{\beta_{21} \in \Theta^{(1)}}{\arg \min } \sum_{t=1}^{n}\left(\epsilon_{2 t}-\beta_{21} \epsilon_{1 t}\right)^{2}
$$

and satisfies

$\sqrt{n}\left(\widehat{\beta}_{21}-\beta_{0,21}\right)=\frac{n^{-1 / 2} \sum_{t=1}^{n} \eta_{2 t} \epsilon_{1 t}}{n^{-1} \sum_{t=1}^{n} \epsilon_{1 t}^{2}} \stackrel{\mathcal{L}}{\rightarrow} \mathcal{N}(0,1)$.

It follows that when $E \eta^{4}$ is large enough, the EbEE of $\beta_{21}$ is asymptotically more accurate than its Full QMLE. By contrast, it can be shown that the EbEE and Full QMLE of $\beta_{32}$ have the same asymptotic distribution.

To illustrate the gain of efficiency of the EbE over the QML in this particular setting, Fig. 1 reports the ratio between the asymptotic variance of the QML estimator of $\beta_{21}$ and its EbE counterpart, i.e. $\frac{1+\beta_{32}^{2} E \eta^{4}}{\left(1+\beta_{32}^{2}\right)^{2}}$, as a function of $\beta_{32}$ and $E \eta^{4}$. We consider values of $\beta_{32}$ ranging between -2 and 2 . Instead of taking different values for $E \eta^{4}$, we assume that $\eta$ follows a standardized Student-t distribution with degree of freedom $v$ such that $E \eta^{4}=3+6 /(v-4)$ for $v>4$.

Fig. 1 confirms that the gain of efficiency of the EbE estimator of $\beta_{21}$ is inversely proportional to $v$ (and therefore increases with $\left.E \eta^{4}\right)$. The loss of efficiency of the Full QMLE can be very large for values of $v<5$, i.e. $E \eta^{4}>9$.

Of course, we considered in the section a particular example, and one can easily find other examples for which the Full QMLE is more efficient than the EbEE.

\subsection{Case where the estimation can be parallelized}

Now consider the CHAR model (2.12)-(2.13). We adapt the notations used in Section 4.1.2 for model (2.10)-(2.11), by setting $\boldsymbol{\theta}^{(i)}=\left(\omega_{i}, \gamma_{i+}, \gamma_{i-}, \alpha_{i}, b_{i}\right)^{\prime}, \quad \varphi^{(i j)}=\left(\varpi_{i j}, \varsigma_{i j+}, \varsigma_{i j-}, \tau_{i j}, \xi_{i j}, c_{i j}\right)^{\prime}$,

for $(i, j) \in \mathfrak{T}_{m}$, keeping the convention of adding the subscript " 0 " to denote the true parameter value. Note that, with this new model, $\widetilde{v}_{i t}\left(\varphi^{(+i)}\right)$ and $\widetilde{g}_{i t}\left(\vartheta^{(+i)}\right)$ depend only on $\varphi^{(i)}$ and $\vartheta^{(i)}$, respectively. Indeed the QMLE is the solution to (4.1), with

$\tilde{q}_{t}(\boldsymbol{\vartheta})=\sum_{i=1}^{m} \tilde{q}_{i t}\left(\boldsymbol{\vartheta}^{(i)}\right)$,

and, for $t=1, \ldots, n$,

$$
\begin{aligned}
\tilde{q}_{i t}\left(\boldsymbol{\vartheta}^{(i)}\right)= & \frac{\widetilde{v}_{i t}^{2}\left(\boldsymbol{\varphi}^{(+i)}\right)}{\widetilde{g}_{i t}\left(\boldsymbol{\vartheta}^{(i)}\right)}+\log \widetilde{g}_{i t}\left(\boldsymbol{\vartheta}^{(i)}\right), \\
\tilde{g}_{i t}\left(\boldsymbol{\vartheta}^{(i)}\right)= & \omega_{i, t-1}+\alpha_{i} \widetilde{v}_{i, t-1}^{2}\left(\boldsymbol{\varphi}^{(i)}\right)+b_{i} \widetilde{g}_{i, t-1}\left(\boldsymbol{\vartheta}^{(i)}\right), \\
\tilde{v}_{i t}\left(\boldsymbol{\varphi}^{(i)}\right)= & \epsilon_{i t}-\sum_{j=1}^{i-1} \widetilde{\beta}_{i j, t}\left(\boldsymbol{\varphi}^{(i)}\right) \epsilon_{j t}, \\
\widetilde{\beta}_{i j, t}\left(\boldsymbol{\varphi}^{(i)}\right)= & \omega_{i j, t-1}+\tau_{i j} \widetilde{v}_{i, t-1}\left(\boldsymbol{\varphi}^{(i)}\right)+\xi_{i j} \widetilde{v}_{i, t-1}\left(\boldsymbol{\varphi}^{(i)}\right) \epsilon_{1, t-1} \\
& +c_{i j} \widetilde{\beta}_{i j, t-1}\left(\boldsymbol{\varphi}^{(i)}\right),
\end{aligned}
$$




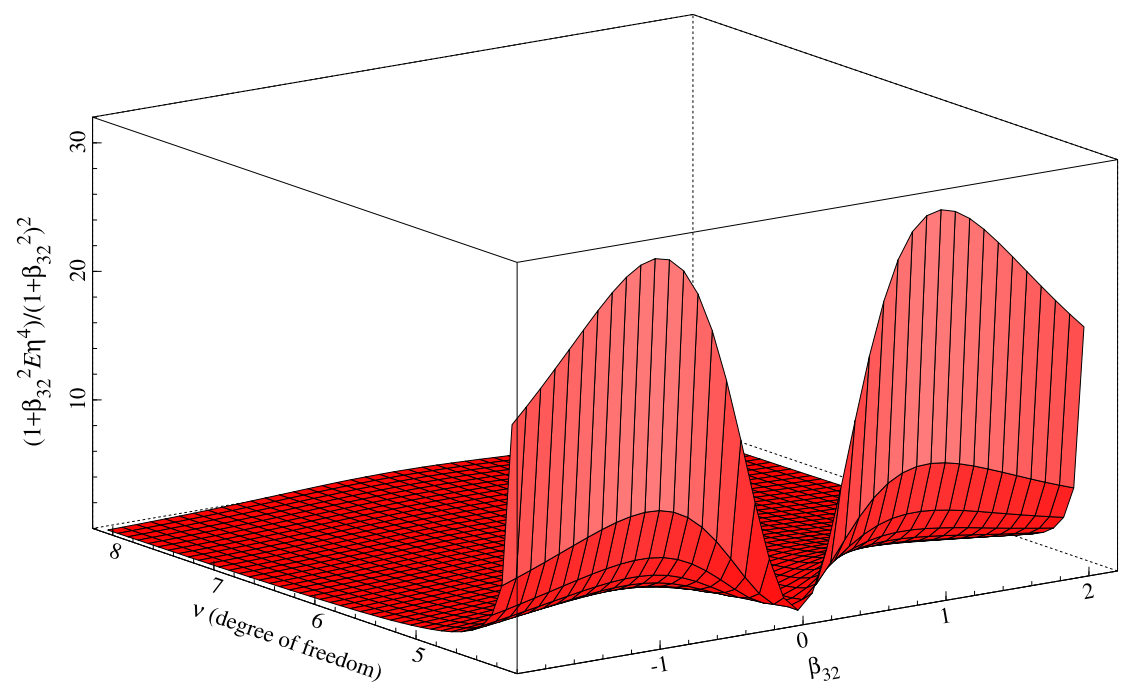

Fig. 1. Ratio between the asymptotic variance of the QML estimator of $\beta_{21}$ and its EbE counterpart as a function of the degree of freedom $v$ and $\beta_{32}$.

and fixed initial values for $\widetilde{\beta}_{i j, 0}\left(\varphi^{(i)}\right), \epsilon_{0}$ and $\widetilde{g}_{i, 0}\left(\vartheta^{(i)}\right)$. The QMLE and EbEE of $\vartheta_{0}^{(i)}$ thus coincide, and can be obtained by solving

$\widehat{\vartheta}_{n}^{(i)}=\underset{\vartheta^{(i)} \in \Theta^{(i)}}{\arg \min } \sum_{t=1}^{n} \widetilde{q}_{i t}\left(\boldsymbol{\vartheta}^{(i)}\right)$,

in parallel (or successively but without any particular order) for all $i \in\{1, \ldots, m\}$. The analog of Theorem 4.2 can be obtained, with a block-diagonal matrix $\Omega$, if the conditions in $\mathbf{B} \mathbf{4}$ are modified in an obvious way, and if the matrix $\boldsymbol{T}$ involved in $\boldsymbol{S}_{t}$ (see (4.9)-(4.11)), with $\ell$ th row $\left(0, \tau_{i j}^{(2)}, \ldots, \tau_{i j}^{(i)}, \mathbf{0}_{m-i}^{\prime}\right)$, is replaced by the matrix $\boldsymbol{T}_{t}$ with $\ell$ th $\operatorname{row}\left(\mathbf{0}_{i-1}^{\prime}, \tau_{i j}+\xi_{i j} \epsilon_{1 t}, \mathbf{0}_{m-i}^{\prime}\right)$.

\subsection{Model based on observed returns instead of factors}

We now consider the estimation of Model (2.15)-(2.16). Note that, contrary to (2.10)-(2.11), the conditional variances depend on past returns instead of past squared factors while conditional betas depend on the product of past returns.

The unknown parameter $\vartheta_{0}$ of this model belongs to a compact set $\Theta$ of $\mathbb{R}^{d}$, with $d=m(7 m+1) / 2$. Suppose that for all generic element $\vartheta$ of $\Theta$, the constraints ensuring the almost sure positivity of $g_{i t}(\vartheta)$ are satisfied: with obvious notation

$\min _{i} \omega_{i}>0 \quad$ and $\quad \min _{i, k}\left\{\alpha_{i+}^{(k)}, \alpha_{i-}^{(k)}, b_{i}\right\} \geq 0 \quad$ for all $\vartheta \in \Theta$.

The fact that $g_{i t}(\boldsymbol{\vartheta})$ and $\beta_{i j, t}(\boldsymbol{\vartheta})$ are now functions of past observations, instead of past factors, inhibits finding explicit stationarity conditions (Theorem 3.1 cannot be applied), but renders easier the asymptotic theory of the QMLE. In particular the invertibility is ensured if

$\left|b_{i}\right|<1 \quad$ and $\quad\left|c_{i j}\right|<1 \quad$ for all $\vartheta \in \Theta$.

To show the consistency of the QMLE, we need to slightly modify B2 and B4. To establish the asymptotic normality, B6 can be replaced by a more explicit condition.

B2*: For $i=1, \ldots, m$, the distributions of $\left(\eta_{i t}^{+}\right)^{2}$ and $\left(\eta_{i t}^{-}\right)^{2}$ conditionally on $\left\{\eta_{j t}, j \neq i\right\}$ are non degenerate. For $i, j=$ $1, \ldots, m$, the distributions of $\eta_{i t} \eta_{j t}$ are non degenerate.

B4*: For $i=1, \ldots, m$, we have $\left(\alpha_{0 i+}^{(1)}, \ldots, \alpha_{0 i-}^{(m)}\right) \neq 0$ and $\tau_{0 i j} \neq 0$.

B6* : We have $E\left\|\boldsymbol{\epsilon}_{t}\right\|^{s_{0}}<\infty$ for some $s_{0}>6$.
Theorem 4.4 (CAN of the QMLE of (2.15)-(2.16)). Let $\left(\boldsymbol{\epsilon}_{t}\right)$ be a stationary ergodic and non anticipative solution to the CHAR model

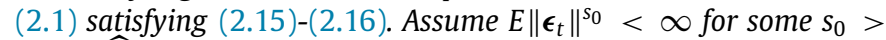
0 . Let $\left(\widehat{\vartheta}_{n}\right)$ be a sequence of QML estimators satisfying (4.1). Under (4.22), (4.23) and $\mathbf{B 2}^{*}, \mathbf{B 4}^{*}$, we have

$\widehat{\vartheta}_{n} \rightarrow \vartheta_{0}, \quad$ almost surely as $n \rightarrow \infty$.

Under the additional assumptions $\mathbf{A 6 ,} \mathbf{A 1 0}$ and $\mathbf{B 6}^{*}$,

$\sqrt{n}\left(\widehat{\vartheta}_{n}-\vartheta_{0}\right) \stackrel{\mathcal{L}}{\rightarrow} \mathcal{N}(0, \Omega), \quad \Omega=J^{-1} \boldsymbol{J}^{-1}$,

where $\mathbf{I}$ and $\mathbf{J}$ are defined in the proof. These two matrices are blockdiagonal when the components of $\boldsymbol{\eta}_{t}$ are independent and symmetrically distributed.

\section{Monte-Carlo simulation}

In this section, we first illustrate the invertibility condition and also emphasize the good finite sample properties of the QML and EbE estimators with a Monte Carlo study.

\subsection{Illustration of the invertibility condition}

To illustrate the importance of the invertibility condition, consider a trivariate stochastic process $\epsilon_{t}=\left(\epsilon_{1 t}, \epsilon_{2 t}, \epsilon_{3 t}\right)^{\prime}=\Sigma_{t}^{1 / 2} \eta_{t}$, compatible with (2.1)-(2.10)-(2.11), with the additional restrictions that $\Sigma_{t}^{1 / 2}=\boldsymbol{B}_{t}^{-1} \boldsymbol{G}_{t}^{1 / 2}$, with $\boldsymbol{G}_{t}=\boldsymbol{G}=\omega_{0} I_{3}, \boldsymbol{\eta}_{t} \stackrel{\text { i.i.d. }}{\sim} \mathcal{N}\left(\mathbf{0}, \boldsymbol{I}_{3}\right)$ and $\beta_{i j, t}=\varpi+\tau_{i j}^{(i)} v_{i, t-1}$ for $(i, j) \in \boldsymbol{T}_{3}$.

This model corresponds to a CHAR model with i.i.d. Gaussian innovations, conditionally homoscedastic orthogonal factors $\left(v_{1 t}, v_{2 t}, v_{3 t}\right)^{\prime}$ and time varying betas depending only on past shocks $v_{i, t-1}$. For simplicity, the constants in the variance of the factors and the conditional betas are restricted to be the same in all equations. The three equations describing the conditional betas can be written more compactly as follows:

$$
\begin{aligned}
\boldsymbol{\beta}_{t} & :=\left(\begin{array}{l}
\beta_{21, t} \\
\beta_{31, t} \\
\beta_{32, t}
\end{array}\right)=\left(\begin{array}{l}
\varpi \\
\varpi \\
\varpi
\end{array}\right)+\left(\begin{array}{ccc}
0 & \tau_{21}^{(2)} & 0 \\
0 & 0 & \tau_{31}^{(3)} \\
0 & 0 & \tau_{32}^{(3)}
\end{array}\right) \boldsymbol{v}_{t-1} \\
& =\left(\begin{array}{l}
\varpi \\
\varpi \\
\varpi
\end{array}\right)+\left(\begin{array}{ccc}
0 & \tau_{21}^{(2)} & 0 \\
0 & 0 & \tau_{31}^{(3)} \\
0 & 0 & \tau_{32}^{(3)}
\end{array}\right)
\end{aligned}
$$



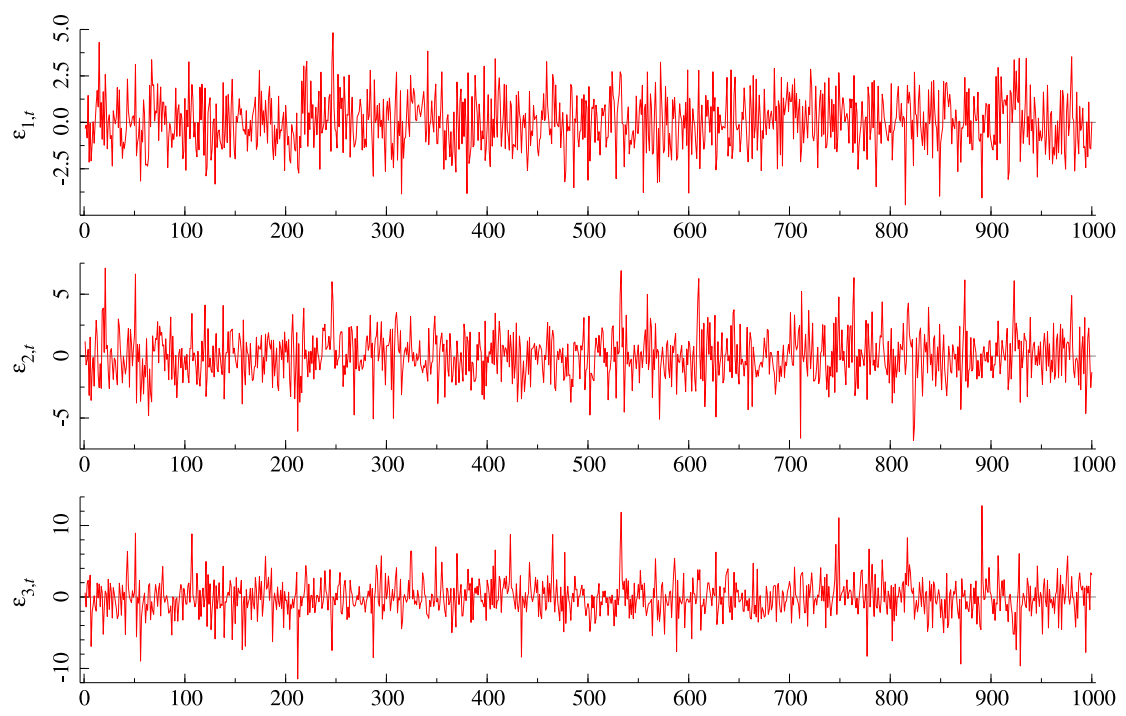

Fig. 2. Simulated returns for the case satisfying the invertibility condition.
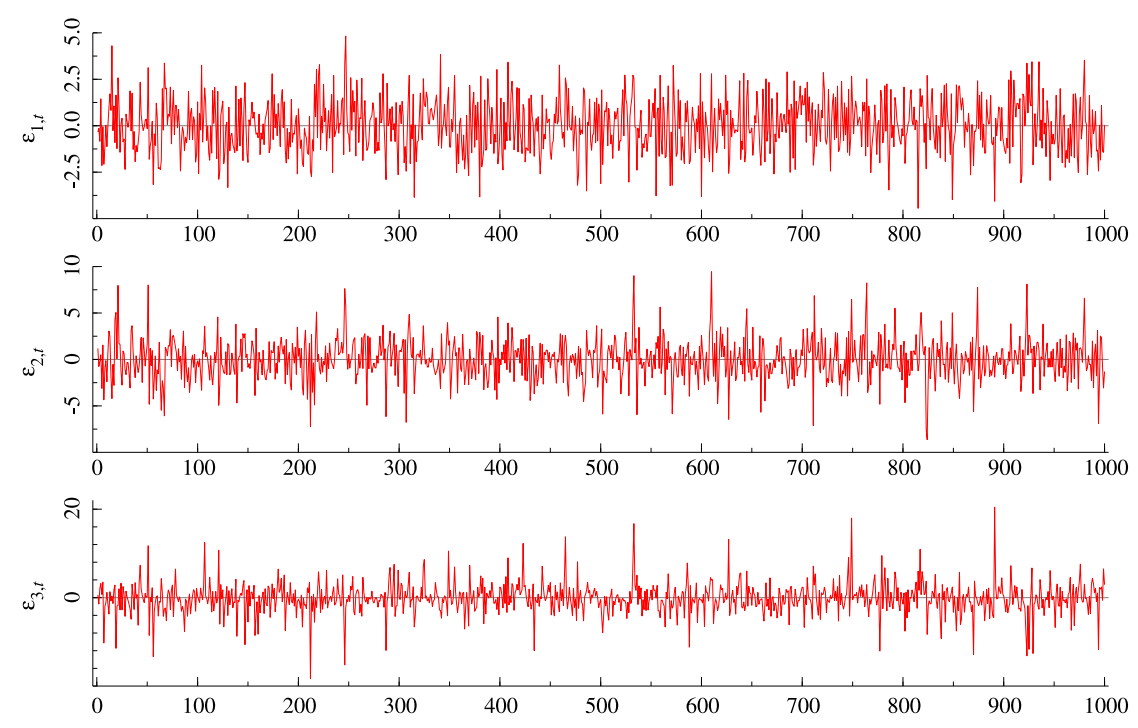

Fig. 3. Simulated returns for the case not satisfying the invertibility condition.

$$
\begin{aligned}
& \times\left(\begin{array}{ccc}
1 & 0 & 0 \\
-\beta_{21, t-1} & 1 & 0 \\
-\beta_{31, t-1} & -\beta_{32, t-1} & 1
\end{array}\right) \boldsymbol{\epsilon}_{t-1} \\
= & \boldsymbol{w}_{t-1}^{*}+\boldsymbol{S}_{t-1} \boldsymbol{\beta}_{t-1},
\end{aligned}
$$

with $\boldsymbol{w}_{t}^{*}=\left(\begin{array}{l}\varpi+\tau_{21}^{(2)} \epsilon_{2, t} \\ \varpi+\tau_{31}^{(3)} \epsilon_{3, t} \\ \varpi+\tau_{32}^{(3)} \epsilon_{3, t}\end{array}\right)$ and $\boldsymbol{S}_{t}=\left(\begin{array}{ccc}-\tau_{21}^{(2)} \epsilon_{1, t} & 0 & 0 \\ 0 & -\tau_{31}^{(3)} \epsilon_{1, t} & -\tau_{31}^{(3)} \epsilon_{2, t} \\ 0 & -\tau_{32}^{(3)} \epsilon_{1, t} & -\tau_{32}^{(3)} \epsilon_{2, t}\end{array}\right)$.

Fig. 2 plots $n=1000$ observations of $\epsilon_{t}$ generated using the above model with parameters $\omega_{0}=2, \varpi=0.5$ and $\tau_{21}^{(1)}=\tau_{31}^{(2)}=$ $\tau_{32}^{(2)}=0.5$.

Filtered betas can be obtained from the simulated log-returns using the following recursive formulas:

$$
\begin{aligned}
v_{k t} & =\epsilon_{k t}-\sum_{j=1}^{k-1} \beta_{k j, t} \epsilon_{j t} \\
\beta_{i j, t} & =\varpi+\tau_{i j}^{(i)} v_{i, t-1}
\end{aligned}
$$

for $k=1,2$ and 3 and $(i, j) \in \boldsymbol{T}_{3}$. Importantly, the parameters $\varpi$ and $\tau_{i j}^{(i)}$ are not estimated but set to their true values and the same initial value $\beta_{i j, 0}=\varpi$ is used in the simulation of the data and in the computation of the betas using the above formulas. Note that this model implies that $\beta_{31, t}=\beta_{32, t} \forall t$ because they both depend only on $v_{3, t-1}$ and furthermore $\tau_{31}^{(2)}=\tau_{32}^{(2)}$.

The invertibility condition stated in (4.11) implies that when $n \rightarrow \infty, \tilde{\gamma}_{S}=\frac{1}{n} \log \left\|\prod_{i=1}^{n} \boldsymbol{S}_{n-i+1}\right\|<0$ for all $\boldsymbol{\varphi} \in \Theta_{\varphi}$ and in particular for ${ }^{n} \varphi_{0}$. Using the Frobenius norm for $\|\cdot\|$, we obtain a value of $\tilde{\gamma}_{S}=-0.41<0$ satisfying the invertibility condition. Note that in this case, filtered and true conditional betas are indistinguishable and therefore not plotted to save space.

Fig. 3 plots 1000 observations generated using the same model as above except that $\tau_{21}^{(1)}=\tau_{31}^{(2)}=\tau_{32}^{(2)}=0.75$. Interestingly, Fig. 3 does not differ dramatically from Fig. 2, except that series 2 and 3 are more volatile and have large peaks in the later case. For this simulation, we have an estimate of $\tilde{\gamma}_{S}=0.0086>0$ implying $\gamma_{S}>0$ (provided that $n$ is sufficiently large), which implies that the invertibility condition is not satisfied in this case. The first two panels of Fig. 4 correspond to filtered values of $\beta_{21, t}$ and $\beta_{31, t}$ using 

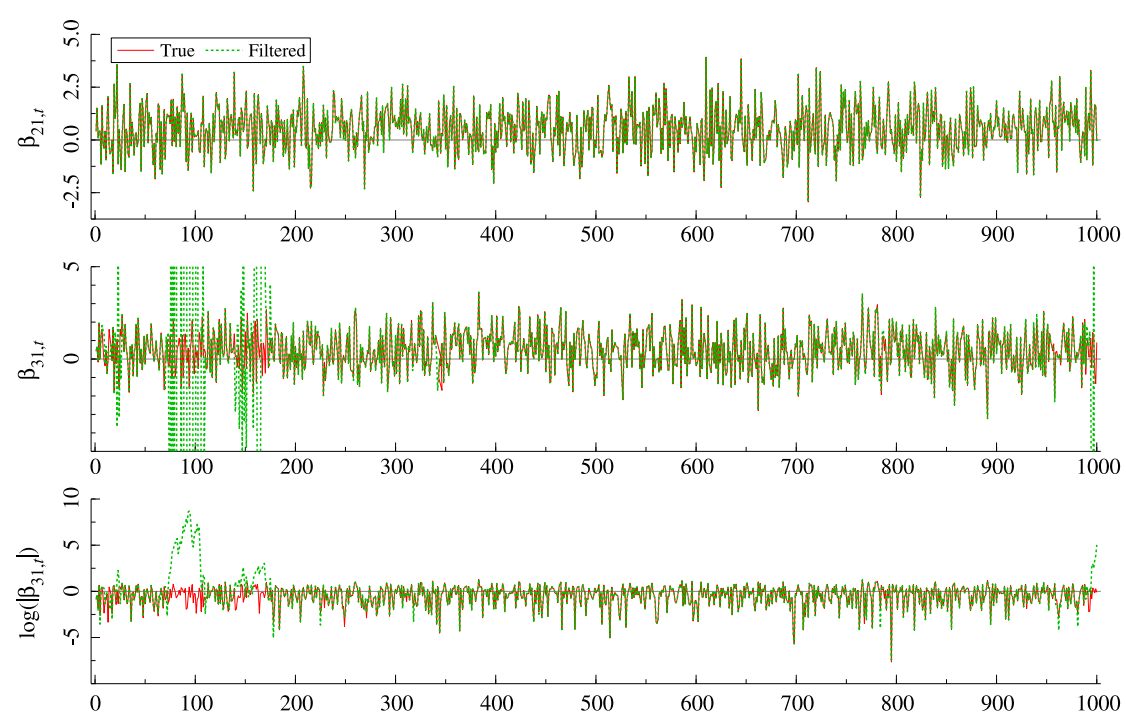

Fig. 4. True and filtered conditional beta for the case not satisfying the invertibility condition.

the above formulas. Recall that the model is specified such that $\beta_{31, t}=\beta_{32, t} \forall t$ and therefore we do not report the graph for $\beta_{32, t}$.

While the filtered values of $\beta_{21, t}$ follow closely the true values, we get a totally different picture for $\beta_{31, t}$. To better visualize the problem, $\log \left(\left|\beta_{31, t}\right|\right)$ and the corresponding filtered values are plotted in the last panel of Fig. 4. Interestingly, the filtered values of $\beta_{31, t}$ deviate completely from the true values for observations $75-100,140-170$ and the end of the sample, which illustrates the impact of the rejection of the invertibility condition.

\subsection{Finite sample properties of the $\mathrm{QML}$ and EbE estimators}

To study the finite sample properties of the QML and EbE estimators of the CHAR model, we perform a Monte Carlo simulation.

Simulation setup: We generate $n=1000,2000$ or 4000 observations of a $m(=5)$-dimensional return process $\epsilon_{t}=\Sigma_{t}^{1 / 2} \eta_{t}$ $(t=1, \ldots, n)$, where $\Sigma_{t}^{1 / 2}=\boldsymbol{B}_{t}^{-1} \boldsymbol{G}_{t}^{1 / 2}$. In the first simulation (i.e., Gaussian case) $\boldsymbol{\eta}_{t} \stackrel{\text { i.i.d. }}{\sim} \mathcal{N}\left(\mathbf{0}, \mathbf{I}_{5}\right)$ while in the second simulation (i.e., Student-t case) innovations follow independent standardized Student-t distributions with 7 degrees of freedom, i.e., $\eta_{t}=$ $\left(\eta_{1 t}, \ldots, \eta_{m t}\right)$, where $\eta_{i t} \stackrel{\text { i.i.d. }}{\sim} T(\mathbf{0}, 1,7)$ for $i=1, \ldots, m$ with $\eta_{i t} \perp \eta_{j t}$ for $i \neq j$.

The respectively $m$ and $m(m-1) / 2$ elements $g_{i t}$ and $\beta_{i j, t}$ defining $\boldsymbol{G}_{t}$ and $\boldsymbol{B}_{t}$ are specified as restricted versions of (2.10)-(2.11), i.e.

$g_{i t}=\omega_{0 i}+\alpha_{0 i} v_{i, t-1}^{2}+b_{0 i} g_{i, t-1}$

$\beta_{i j, t}=\varpi_{0 i j}+\tau_{0 i j} v_{i, t-1}+c_{0 i j} \beta_{i j, t-1}$.

We set the parameters governing the dynamics in the conditional variances to $\omega_{0 i}=0.1, \alpha_{0 i}=0.1$ and $b_{0 i}=0.8$ (for $i=1, \ldots, M)$ so that all elements of $\boldsymbol{v}_{t}$ have a unit unconditional variance and GARCH effects. Parameters of the conditional betas are set to $\varpi_{0 i j}=0.1 \tau_{0 i j}=0.2$ and $c_{0 i j}=0.8$. Results reported below are based on programs written by the authors using $\mathrm{Ox}$ version 7.1 (Doornik, 2012).

Results: Summary statistics on the Monte Carlo simulation are reported in Tables 1 and 2. The left part of both tables corresponds to the Full QMLE while the right part is for the EbEE. Due to the high number of parameters, results are not reported for each parameter separately. Instead, we report averages across the number of series for the GARCH parameters entering in $\boldsymbol{\theta}$ (i.e., $\omega, \alpha$ and $\beta$ ) or across the number of conditional betas for the parameters entering in $\varphi$ (i.e., $\varpi, \tau$ and $c$ ). Note that for $\omega, \alpha$ and $\beta$, averages are taken over $m=5$ values while for $\varpi, \tau$ and $c$, they are taken over $m(m-1) / 2=10$ values. Averages across all parameters are also reported in rows labeled "ALL"

Columns "BIAS" correspond to the empirical bias of the estimates over 1000 replications. More specifically, the biases reported in rows " $\omega$ " are computed as follows:

BIAS of $\hat{\omega}=\frac{1}{1000} \sum_{r=1}^{1000} \frac{1}{5} \sum_{i=1}^{5}\left(\hat{\omega}_{i}^{(r)}-\omega_{0 i}\right)$,

where $\hat{\omega}_{i}^{(r)}$ is either the Full QMLE or EbEE of $\omega_{i}$ obtained at the $r$ th replication. Similarly, the RMSE of $\hat{\omega}$ is computed as follows:

RMSE of $\hat{\omega}=\sqrt{\frac{1}{1000} \sum_{r=1}^{1000} \frac{1}{5} \sum_{i=1}^{5}\left(\hat{\omega}_{i}^{(r)}-\omega_{0 i}\right)^{2}}$.

The relative efficiency of the EbEE with respect to the Full QMLE is reported in column "RE". For each row of the tables, RE is defined as the ratio between the RMSE of the EbE divided by the RMSE of the Full QML. A value of RE greater than 1 therefore means that the Full QML is more efficient than the EbE. Finally, columns " $5 \%$ $C B$ " and " $95 \%$ CB" correspond to the frequencies of true parameters falling below the bands of a $90 \%$ confidence interval computed using Theorems 4.2 and 4.3, respectively for the Full QMLE and EbEE. Again these frequencies are averaged over the number of GARCH equations or conditional betas.

Results suggest that both the Full QML and EbE deliver satisfactory results in the sense that biases are very small for all parameters (particularly for $n=2000$ and 4000). In the Gaussian case (i.e. Table 1), the Full QML is about 9\%, 16\% and 30\% more efficient than the EbE, respectively for $n=1000,2000$ and 4000. In the case of Student-t, innovations the RE of the Full QML is slightly greater.

About the validity of Theorems 4.2 and 4.3 in finite samples, the rejection frequencies reported in columns " $5 \%$ CB" and " $95 \%$ $C B$ " are close to the theoretical values for all parameters both for Gaussian and Student-t innovations.

Unreported simulation results (available upon request) suggest very similar finite sample behaviors of the Full and EbE QML for a specification of the conditional betas of the type $\beta_{i j, t}=\varpi_{0 i j}+$ $\tau_{0 i j} v_{j, t-1}+c_{0 i j} \beta_{i j, t-1}, \beta_{i j, t}=\varpi_{0 i j}+\tau_{0 i j} v_{i, t-1} v_{1, t-1}+c_{0 i j} \beta_{i j, t-1}$ and $\beta_{i j, t}=\varpi_{0 i j}+\tau_{0 i j} v_{i, t-1} v_{j, t-1}+c_{0 i j} \beta_{i j, t-1}$. The first two specifications 
Table 1

Monte-Carlo simulation - Gaussian case.

\begin{tabular}{|c|c|c|c|c|c|c|c|c|c|}
\hline & \multicolumn{4}{|l|}{ Full QML } & \multicolumn{4}{|l|}{$\mathrm{EbE}$} & \multirow[t]{2}{*}{$\mathrm{RE}$} \\
\hline & BIAS & RMSE & $5 \%$ CB & $95 \%$ CB & BIAS & RMSE & $5 \%$ CB & $95 \% \mathrm{CB}$ & \\
\hline \multicolumn{10}{|c|}{$n=1000$} \\
\hline$\omega$ & 0.0160 & 0.0614 & 3.5988 & 93.4440 & 0.0190 & 0.0672 & 4.070 & 92.251 & 1.10 \\
\hline$\alpha$ & 0.0030 & 0.0295 & 3.2265 & 92.9470 & 0.0036 & 0.0322 & 3.659 & 91.696 & 1.09 \\
\hline$\beta$ & -0.0196 & 0.0804 & 6.2461 & 95.1600 & -0.0230 & 0.0876 & 7.379 & 94.347 & 1.09 \\
\hline$\varpi$ & 0.0006 & 0.0121 & 4.2192 & 93.4950 & 0.0007 & 0.0132 & 4.604 & 92.559 & 1.08 \\
\hline$\tau$ & 0.0009 & 0.0149 & 5.6256 & 95.2640 & 0.0011 & 0.0165 & 6.208 & 94.779 & 1.10 \\
\hline c & -0.0013 & 0.0230 & 6.1531 & 95.3980 & -0.0016 & 0.0250 & 7.009 & 94.913 & 1.09 \\
\hline ALL & 0.0000 & 0.0301 & 5.0075 & 94.4300 & 0.0000 & 0.0329 & 5.639 & 93.644 & 1.09 \\
\hline \multicolumn{10}{|c|}{$n=2000$} \\
\hline$\omega$ & 0.0068 & 0.0320 & 2.4869 & 95.4020 & 0.0087 & 0.0370 & 3.305 & 93.138 & 1.16 \\
\hline$\alpha$ & 0.0013 & 0.0185 & 2.6750 & 95.2560 & 0.0017 & 0.0217 & 3.766 & 93.410 & 1.17 \\
\hline$\beta$ & -0.0082 & 0.0439 & 4.2633 & 96.6140 & -0.0104 & 0.0510 & 6.130 & 95.460 & 1.16 \\
\hline$\varpi$ & 0.0001 & 0.0067 & 3.8454 & 94.5140 & 0.0002 & 0.0079 & 4.550 & 93.410 & 1.17 \\
\hline$\tau$ & 0.0002 & 0.0088 & 4.6290 & 95.6010 & 0.0004 & 0.0102 & 5.690 & 94.812 & 1.17 \\
\hline c & -0.0003 & 0.0126 & 5.0888 & 96.2800 & -0.0004 & 0.0148 & 6.266 & 95.690 & 1.18 \\
\hline ALL & 0.0000 & 0.0167 & 4.0613 & 95.5630 & 0.0000 & 0.0195 & 5.135 & 94.426 & 1.17 \\
\hline \multicolumn{10}{|c|}{$n=4000$} \\
\hline$\omega$ & 0.0024 & 0.0172 & 2.1475 & 96.3990 & 0.0039 & 0.0225 & 3.442 & 93.831 & 1.31 \\
\hline$\alpha$ & 0.0005 & 0.0113 & 2.2993 & 96.8980 & 0.0006 & 0.0148 & 3.831 & 94.372 & 1.30 \\
\hline$\beta$ & -0.0030 & 0.0248 & 3.4924 & 97.2450 & -0.0046 & 0.0321 & 5.649 & 95.909 & 1.29 \\
\hline$\varpi$ & 0.0000 & 0.0037 & 2.8850 & 96.1710 & 0.0000 & 0.0049 & 4.058 & 94.177 & 1.31 \\
\hline$\tau$ & 0.0001 & 0.0049 & 3.4924 & 96.7140 & 0.0001 & 0.0065 & 5.336 & 95.249 & 1.33 \\
\hline c & -0.0001 & 0.0071 & 3.5792 & 96.9310 & -0.0001 & 0.0092 & 5.487 & 95.747 & 1.30 \\
\hline ALL & 0.0000 & 0.0094 & 3.0947 & 96.6860 & 0.0000 & 0.0123 & 4.743 & 94.940 & 1.30 \\
\hline
\end{tabular}

Note: Monte-Carlo simulation results based on 1000 replications. DGP is a $m(=5)$-dimensional return process $\boldsymbol{\epsilon}_{t}=$ $\Sigma_{t}^{1 / 2} \boldsymbol{\eta}_{t}$, where $\Sigma_{t}^{1 / 2}=\boldsymbol{B}_{t}^{-1} \boldsymbol{G}_{t}^{1 / 2}, \boldsymbol{\eta}_{t} \stackrel{\text { i.i.d. }}{\sim} \mathcal{N}\left(\mathbf{0}, \mathbf{I}_{5}\right), g_{i t}=\omega_{0 i}+\alpha_{0 i} v_{i, t-1}^{2}+b_{0 i} g_{i, t-1}\left(\right.$ with $\left.\omega_{0 i}=0.1, \alpha_{0 i}=0.1, b_{0 i}=0.8\right)$ for $i=1, \ldots, m, \beta_{i j, t}=\varpi_{0 i j}+\tau_{0 i j} v_{i, t-1}+c_{0 i j} \beta_{i j, t-1}\left(\right.$ with $\varpi_{0 i j}=0.1 \tau_{0 i j}=0.2$ and $\left.c_{0 i j}=0.8\right)$ for $i=1, \ldots, m, j<i$. Columns "BIAS" and "RMSE" correspond to the empirical bias and root mean square error. Columns " $5 \%$ CB" and " $95 \%$ CB" correspond to the frequencies of true parameters falling below the bands of a $90 \%$ confidence interval. Column "RE" is the ratio between the RMSE of the EbE and that of Full QML. Entries in this table are averages across the $m$ series for $\omega, \alpha$ and $\beta$, across the $m(m-1) / 2$ conditional betas for the parameters $\varpi, \tau$ and $c$ and across all parameters for rows labeled "All".

Table 2

Monte-Carlo simulation - Student-t case.

\begin{tabular}{|c|c|c|c|c|c|c|c|c|c|}
\hline & \multicolumn{4}{|l|}{ Full QML } & \multicolumn{4}{|l|}{$\mathrm{EbE}$} & \multirow[t]{2}{*}{ RE } \\
\hline & BIAS & RMSE & $5 \% \mathrm{CB}$ & $95 \% \mathrm{CB}$ & BIAS & RMSE & $5 \% \mathrm{CB}$ & $95 \% \mathrm{CB}$ & \\
\hline \multicolumn{10}{|c|}{$n=1000$} \\
\hline$\omega$ & 0.0179 & 0.0689 & 3.3720 & 92.4130 & 0.0225 & 0.0771 & 4.227 & 90.284 & 1.12 \\
\hline$\alpha$ & 0.0039 & 0.0355 & 2.2339 & 91.9070 & 0.0057 & 0.0414 & 2.860 & 90.074 & 1.17 \\
\hline$\beta$ & -0.0233 & 0.0919 & 6.9968 & 94.5840 & -0.0293 & 0.1031 & 8.980 & 93.270 & 1.12 \\
\hline$\varpi$ & 0.0008 & 0.0135 & 4.0569 & 93.9520 & 0.0010 & 0.0144 & 4.532 & 92.650 & 1.06 \\
\hline$\tau$ & 0.0010 & 0.0155 & 4.9104 & 95.9750 & 0.0012 & 0.0178 & 5.910 & 95.152 & 1.15 \\
\hline$c$ & -0.0016 & 0.0245 & 5.9536 & 95.7960 & -0.0018 & 0.0271 & 7.308 & 95.226 & 1.10 \\
\hline ALL & -0.0001 & 0.0337 & 4.7161 & 94.4830 & -0.0001 & 0.0378 & 5.730 & 93.298 & 1.12 \\
\hline \multicolumn{10}{|c|}{$n=2000$} \\
\hline$\omega$ & 0.0064 & 0.0337 & 2.1758 & 94.7250 & 0.0093 & 0.0416 & 3.141 & 91.734 & 1.23 \\
\hline$\alpha$ & 0.0009 & 0.0215 & 1.7363 & 93.8460 & 0.0020 & 0.0266 & 2.748 & 91.189 & 1.24 \\
\hline$\beta$ & -0.0080 & 0.0478 & 5.0989 & 96.7470 & -0.0120 & 0.0588 & 7.546 & 94.984 & 1.23 \\
\hline$\varpi$ & 0.0002 & 0.0065 & 3.0659 & 95.7030 & 0.0003 & 0.0080 & 4.308 & 94.079 & 1.22 \\
\hline$\tau$ & 0.0003 & 0.0083 & 3.6813 & 96.6810 & 0.0005 & 0.0101 & 4.984 & 95.507 & 1.22 \\
\hline$c$ & -0.0005 & 0.0124 & 4.2088 & 96.9120 & -0.0007 & 0.0150 & 5.965 & 95.725 & 1.21 \\
\hline ALL & -0.0001 & 0.0175 & 3.4359 & 95.9900 & 0.0000 & 0.0215 & 4.883 & 94.281 & 1.23 \\
\hline \multicolumn{10}{|c|}{$n=4000$} \\
\hline$\omega$ & 0.0023 & 0.0179 & 1.7156 & 97.0390 & 0.0049 & 0.0259 & 3.125 & 93.310 & 1.45 \\
\hline$\alpha$ & 0.0002 & 0.0123 & 1.3631 & 96.6630 & 0.0008 & 0.0177 & 3.194 & 92.755 & 1.44 \\
\hline$\beta$ & -0.0028 & 0.0259 & 3.0552 & 97.7910 & -0.0060 & 0.0374 & 6.505 & 95.370 & 1.44 \\
\hline$\varpi$ & 0.0001 & 0.0034 & 2.8555 & 96.7100 & 0.0001 & 0.0050 & 4.630 & 94.051 & 1.46 \\
\hline$\tau$ & 0.0000 & 0.0045 & 2.7850 & 97.4150 & 0.0002 & 0.0065 & 4.792 & 95.567 & 1.45 \\
\hline$c$ & -0.0002 & 0.0064 & 3.4078 & 97.1210 & -0.0002 & 0.0094 & 5.787 & 95.486 & 1.46 \\
\hline ALL & 0.0000 & 0.0094 & 2.6923 & 97.1090 & 0.0000 & 0.0136 & 4.805 & 94.627 & 1.45 \\
\hline
\end{tabular}

Note: See Table 1 expect that $\eta_{t}=\left(\eta_{1 t}, \ldots, \eta_{m t}\right)$, where $\eta_{i t} \stackrel{\text { i.i.d. }}{\sim} T(\mathbf{0}, 1,7)$ for $i=1, \ldots, m$ with $\eta_{i t} \perp \eta_{j t}$ for $i \neq j$.

are nested respectively in (2.11) and (2.13). The third one satisfies the stationarity conditions stated in Section 3. When $m>2$ it is not embedded in the theoretical setting of Section 4 but this specification is found to be empirically relevant (see next section).

\section{Empirical applications}

The aim of this section is to illustrate the usefulness of the CHAR model in a portfolio and risk management exercise. We 

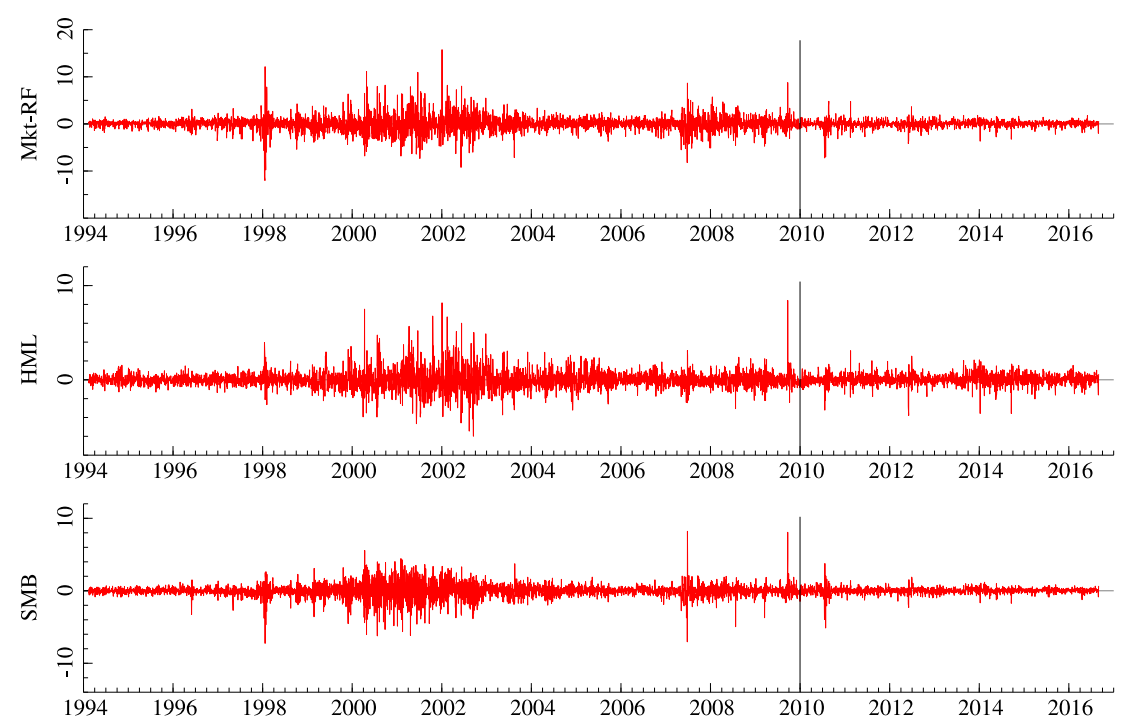

Fig. 5. Time series graph of the three factors $\left(M K T_{t}, S M B_{t}\right.$ and $\left.H M L_{t}\right)$ for the whole sample (i.e., in- and out-of-sample period).
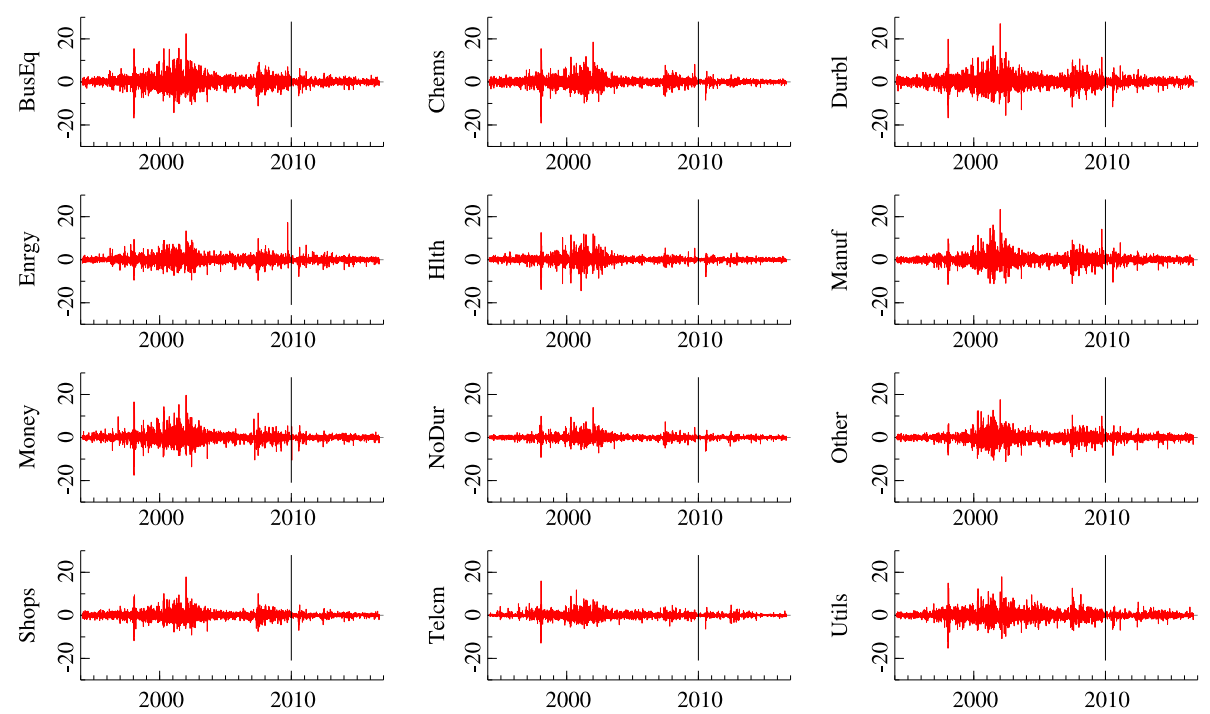

Fig. 6. Time series graph of the log-returns of the 12 industry portfolios for the whole sample (i.e., in- and out-of-sample period).

start with the same dataset used in Engle (2016). We consider the 12 US industry portfolios (i.e., BusEq, Chems, Durbl, Enrgy, Hlth, Manuf, Money, NoDur, Other, Shops, Telcm and Utils) and the three factors introduced in an asset pricing context by Fama and French (1992, 2004). Daily data are obtained from Ken French's website Database available at http://mba.tuck.dartmouth.edu/pages/ faculty/ken.french/data_library.html and cover the period 1994August 2016. The three risk factors are the market factor MKT proxied by the excess log-returns on the SP500 index as well as the standard Fama-French size and value factors SMB (Small Minus Big) and HML (High Minus Low). The 3 factors $M K T_{t}, S M B_{t}, H M L_{t}$ and the 12 industry portfolios log-returns in excess to the risk free rate (i.e., $r_{k t}$ for $k=1, \ldots, 12$ ) are plotted in Figs. 5 and 6 , respectively. All the familiar asset pricing results can be easily expressed in terms of conditional betas, and in particular

$$
\begin{aligned}
E_{t-1}\left(r_{k t}\right)= & \beta_{k, M K T, t} E_{t-1}\left(M K T_{t}\right)+\beta_{k, S M B, t} E_{t-1}\left(S M B_{t}\right) \\
& +\beta_{k, H M L, t} E_{t-1}\left(H M L_{t}\right),
\end{aligned}
$$

where $\beta_{k, M K T, t}, \beta_{k, S M B, t}$ and $\beta_{k, H M L, t}$ are the three time-varying parameters of interest to be estimated from the data. In this asset pricing context, expected returns on any asset is linear in the betas and only depend upon the risk premiums embedded in the factors. In other words, there is no alpha or intercept in (6.1). This property can be directly used to build an in-sample test of the asset pricing model (see e.g., Engle, 2016).

We follow the approach initially proposed by Patton and Verardo (2012) that consists in building hedged portfolios to offset some unwanted exposures to predetermined factors. Let us consider an industry portfolio with a clear small caps bias. The over/underperformance of this portfolio compared to the market can be explained by a statistically significant $\beta_{k, S M B, t}$ coefficient. The only way to control for this effect is to build a portfolio that buys the industry portfolio and sells the implied SMB exposure. If $\beta_{k, S M B, t}$ is time-varying, out-of-sample forecasts are needed to adjust the position. Generalizing this idea to the three factors, Eq. (6.1) leads to an hedged portfolio or equivalently a tracking error series with zero mean.

To implement this strategy, four competing models are used to estimate the three conditional betas and obtain one-step-ahead forecasts, i.e., two DCB models and two CHAR models. For the DCB models, we consider a CCC-GARCH $(1,1)$ model and a DCC$\operatorname{GARCH}(1,1)$ estimated on 4-dimensional systems $\boldsymbol{\epsilon}_{t}=\left(\mathbf{x}_{t}, y_{t}\right)^{\prime}$ 
Table 3

Full and EbE QMLE of the CHAR model for BusEq.

\begin{tabular}{|c|c|c|c|c|c|c|}
\hline & \multicolumn{3}{|l|}{ Full QML } & \multicolumn{3}{|l|}{ EbE QML } \\
\hline & Coefficient & Std-Err & $p$-value & Coefficient & Std-Err & $p$-value \\
\hline$\omega_{1}$ & 0.01924 & 0.00495 & 0.00010 & 0.01924 & 0.00495 & 0.00010 \\
\hline$\alpha_{1}$ & 0.09558 & 0.01134 & 0.00000 & 0.09558 & 0.01134 & 0.00000 \\
\hline$b_{1}$ & 0.89050 & 0.01184 & 0.00000 & 0.89050 & 0.01184 & 0.00000 \\
\hline$\omega_{2}$ & 0.00575 & 0.00173 & 0.00090 & 0.00575 & 0.00173 & 0.00090 \\
\hline$\alpha_{2}$ & 0.06644 & 0.01176 & 0.00000 & 0.06644 & 0.01176 & 0.00000 \\
\hline$b_{2}$ & 0.91329 & 0.01574 & 0.00000 & 0.91329 & 0.01573 & 0.00000 \\
\hline$\varpi_{21}$ & 0.00057 & 0.00043 & 0.18980 & 0.00058 & 0.00043 & 0.17750 \\
\hline$\tau_{21}$ & 0.02128 & 0.00334 & 0.00000 & 0.02128 & 0.00305 & 0.00000 \\
\hline$c_{21}$ & 0.99620 & 0.00162 & 0.00000 & 0.99621 & 0.00160 & 0.00000 \\
\hline$\omega_{3}$ & 0.00163 & 0.00071 & 0.02160 & 0.00163 & 0.00071 & 0.02070 \\
\hline$\alpha_{3}$ & 0.08838 & 0.01965 & 0.00000 & 0.08838 & 0.01942 & 0.00000 \\
\hline$b_{3}$ & 0.90699 & 0.02020 & 0.00000 & 0.90699 & 0.01996 & 0.00000 \\
\hline$\varpi_{31}$ & 0.00004 & 0.00015 & 0.79040 & 0.00003 & 0.00015 & 0.85730 \\
\hline$\tau_{31}$ & 0.02336 & 0.00779 & 0.00270 & 0.02336 & 0.00689 & 0.00070 \\
\hline$c_{31}$ & 0.99546 & 0.00150 & 0.00000 & 0.99547 & 0.00148 & 0.00000 \\
\hline$\varpi_{32}$ & -0.24824 & 0.06639 & 0.00020 & -0.24824 & 0.07138 & 0.00050 \\
\hline$\tau_{32}$ & 0.09610 & 0.01117 & 0.00000 & 0.09610 & 0.01076 & 0.00000 \\
\hline$c_{32}$ & -0.30902 & 0.31845 & 0.33190 & -0.30902 & 0.35103 & 0.37870 \\
\hline$\omega_{4}$ & 0.00106 & 0.00060 & 0.07700 & 0.00106 & 0.00061 & 0.07940 \\
\hline$\alpha_{4}$ & 0.04480 & 0.01468 & 0.00230 & 0.04480 & 0.01481 & 0.00250 \\
\hline$b_{4}$ & 0.95173 & 0.01554 & 0.00000 & 0.95173 & 0.01566 & 0.00000 \\
\hline$\varpi_{41}$ & 0.00394 & 0.00140 & 0.00480 & 0.00394 & 0.00143 & 0.00600 \\
\hline$\tau_{41}$ & 0.01036 & 0.00223 & 0.00000 & 0.01036 & 0.00223 & 0.00000 \\
\hline$c_{41}$ & 0.99630 & 0.00126 & 0.00000 & 0.99630 & 0.00130 & 0.00000 \\
\hline$\varpi_{42}$ & 0.08221 & 0.01696 & 0.00000 & 0.08221 & 0.01734 & 0.00000 \\
\hline$\varpi_{43}$ & -0.00242 & 0.00176 & 0.16800 & -0.00242 & 0.00192 & 0.20820 \\
\hline$\tau_{43}$ & 0.04332 & 0.02636 & 0.10040 & 0.04332 & 0.02665 & 0.10420 \\
\hline$c_{43}$ & 0.99509 & 0.00365 & 0.00000 & 0.99509 & 0.00405 & 0.00000 \\
\hline
\end{tabular}

Note: Full QMLE (left panel) and EbE QMLE (right panel) of the CHAR model for BusEq and the period spanning from $2000-$ 02-08 to 2015-12-31. Standard errors are computed using the formulas described in Theorems 4.2 and Theorem 4.3.

with $\mathbf{x}_{t}=\left(M K T_{t}, S M B_{t}, H M L_{t}\right)^{\prime}$ and $y_{t}=r_{k t}$ using the same notation as in Section 2. In-sample conditional beta estimates of the DCB models are obtained using the formula $\boldsymbol{\Sigma}_{y x, t} \boldsymbol{\Sigma}_{x x, t}^{-1}$ while one-step-ahead forecasts are obtained as $\boldsymbol{\Sigma}_{y x, t+1 \mid t} \boldsymbol{\Sigma}_{x x, t+1 \mid t}^{-1}$.

The third model is a CHAR model with constant betas (denoted $\mathrm{C}-\mathrm{CHAR}$ ) and a $\mathrm{GARCH}(1,1)$ specification for the conditional variance of the factors, i.e.,

$g_{i, t}=\omega_{i}+\alpha_{i} v_{i, t-1}^{2}+b_{i} g_{i, t-1}, \quad \beta_{i j, t}=\varpi_{i j}$.

As explained above, the order of the series might matter in Cholesky decompositions. In our case, the last series of the vector $\boldsymbol{\epsilon}_{t}$ is known and corresponds to the $k$ th industry portfolio so that $\beta_{41, t}$, $\beta_{42, t}$ and $\beta_{43, t}$ correspond to $\beta_{k, M K T, t}, \beta_{k, S M B, t}$ and $\beta_{k, H M L, t}$ (in a certain order). The choice of $\epsilon_{1, t}$ is also natural because it makes more sense to dynamically orthogonalize the $S M B$ and the $H M L$ factors with respect to the $M K T$ factor rather than the opposite. Indeed, the size and value factors are generated using long/short dollar neutral strategies to get approximatively market neutral returns. The order between SMB and HML is however not known apriori and has been chosen by maximizing the empirical log-likelihood. We fixed the order as follows: $\boldsymbol{\epsilon}_{t}=\left(M K T_{t}, S M B_{t}, H M L_{t}, r_{k t}\right)^{\prime}$.

The last model (simply denoted CHAR) relaxes the assumption of constancy of the conditional betas. We start by discussing the specification of the conditional betas of the first three components of $\boldsymbol{\epsilon}_{t}$, as they are identical whatever the industry portfolio we consider. We tested several specifications for the $\beta_{i j, t}$ 's and found that the best one (relying on t-tests, Wald tests and information criteria) is

$\beta_{i j, t}=\varpi_{i j}+\tau_{i j} v_{i, t-1} v_{j, t-1}+c_{i j} \beta_{i j, t-1}$

for $(i, j)$ belonging to the set $\boldsymbol{T}_{3}{ }^{8}$ With this choice, we can empirically check for instance whether the factors are already

\footnotetext{
8 The $\beta_{i j, t}$ 's do not need to be constrained to be positive and therefore can depend on variables taking values on $\Re$. Our theoretical framework allows to include past
}

orthogonal on average (i.e, $\varpi_{i j}=0$ ), or need to be conditionally orthogonalized (i.e, $\varpi_{i j}, \tau_{i j}, c_{i j} \neq 0$ ).

In a second step, and to model the remaining $\beta_{k, M K T, t}, \beta_{k, S M B, t}$ and $\beta_{k, H M L, t}$ terms relative to a given industry portfolio, several specifications are considered. We allow each conditional beta to be either constant like in (6.2) or time-varying according to (6.3). The best specification (chosen using the SIC) is used to obtain onestep ahead forecasts of the three conditional betas of interest. Note that in almost all cases, the best model is found to be the one where all conditional betas follow Eq. (6.3). One-step-ahead forecasts, denoted $\beta_{i j, t+1 \mid t}$, are obtained using either (6.2) or by translating (6.3) one step into the future because (6.3) only depends on quantities that are observed at time $t$.

The first two models are estimated by Gaussian QML while the two CHAR models are estimated by the EbE QMLE described in Section 4. All models are estimated on demeaned log-returns (the empirical means are computed on in-sample observations and not the whole sample) on rolling windows of 4000 observations. Models are reestimated every 3 steps (parameters are therefore kept constant to produce three consecutive forecasts before being reevaluated).

Before presenting the results of the forecasting exercise, we report in Table 3 the estimation results of a CHAR model for series BusEq and the period spanning from 2000-02-08 to 2015-12-31, i.e., the values used to produce the forecasts of the CHAR model for the first working day in 2016. The left part of the table corresponds to the Full QMLE and the right part to the EbE QMLE (i.e., the method used to produce the forecasts). We choose to comment this example because it is one of the few cases where not all conditional betas are time-varying.

values of the $i$ first factors and also to test an asymmetry in the response to positive and negative shocks of the first series (i.e., Market). It turns out that none of these variables are found to be significant. 

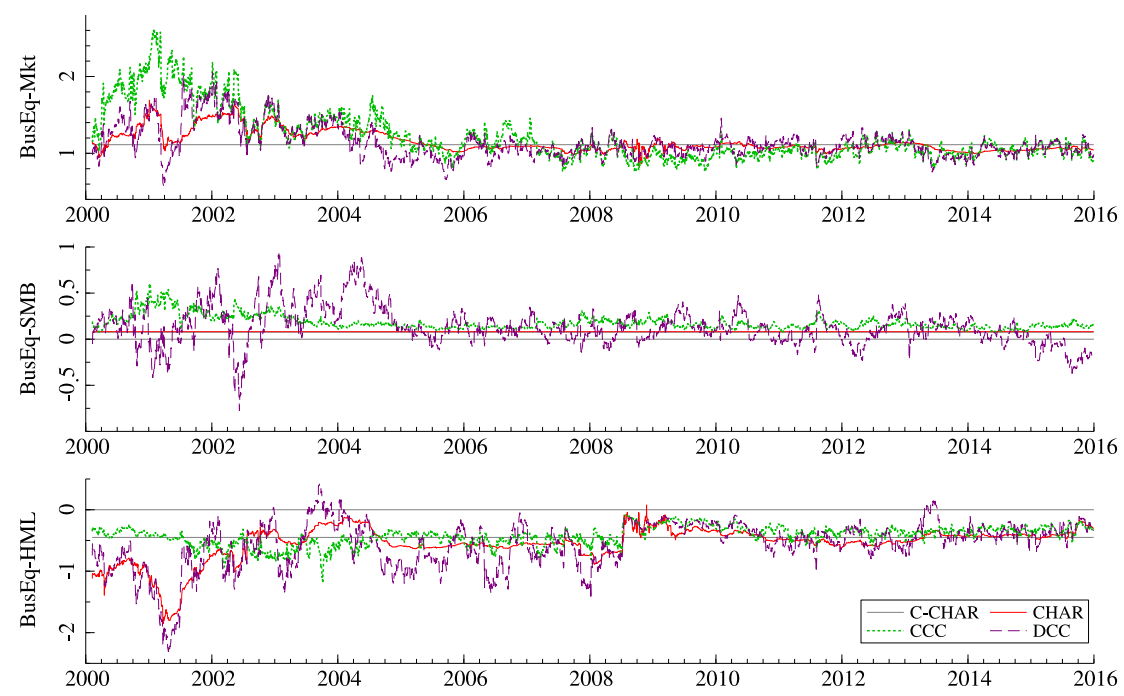

Fig. 7. Conditional betas ( $\beta_{B u s E q, M K T, t}, \beta_{B u s E q, S M B, t}$ and $\beta_{B u s E q, H M L, t}$ ) of the four competing models for the period spanning from 2000-02-08 to 2015-12-31.

Importantly, results of the two estimation methods are found to be almost identical, reason why we rely on the EbE estimator in the forecasting exercise because convergence is achieved much faster with this multi-step method. First, Table 3 suggests that all factors have GARCH effects as the $\alpha_{i}$ and $b_{i}, i=1, \ldots, 4$ coefficients are statistically significant. Second, the two $S M B$ and $H M L$ factors are marginally orthogonal to the $M K T$ factor as the parameters $\varpi_{21}$ and $\varpi_{31}$ are not statistically significant and the estimates of the unconditional betas are 0.15 and -0.19 , respectively for $E\left(\beta_{21, t}\right)$ and $E\left(\beta_{31, t}\right)$. However, the two corresponding conditional market betas show a time-varying behavior with long term persistence (the two autoregressive coefficients $c_{21}$ and $c_{31}$ are close to 1 ), meaning that we can use current information to predict non zero conditional market betas for the value and size factors. Concerning the projection of $H M L$ on the $S M B$ factor, we observe a negative marginal value but a simpler time-varying behavior with no long term persistence as the $c_{3,2}$ parameter is not statistically different from zero. This last result on $S M B$ and $H M L$ justifies our choice to orthogonalize the three factors. Third, we now study the dynamic properties of the industry portfolio returns. While $\beta_{B u s E q, S M B, t}$ is constant and slightly positive $\left(\beta_{42, t}=\varpi_{42}=0.08\right)$, time-varying behavior is detected in $\beta_{B u s E q, M K T, t}$ and $\beta_{B u s E q, H M L, t}$, and found to be very persistent (with estimates of the autoregressive coefficients $c_{41}$ and $c_{43}$ again close to 1 ).

The estimated conditional betas $\beta_{B u s E q, M K T, t}, \beta_{B u s E q, S M B, t}$ and $\beta_{B u s E q, H M L, t}$ are plotted in Fig. 7. Each graph contains the estimated betas for the four competing models. It is interesting to notice that conditional betas filtered with the CHAR model are much smoother than those obtained with the DCC-DCB model. In the case of $\beta_{B U s E q, M K T, t}$ for example, we expect to get a conditional beta stable and close to 1 . We observe this result for all models, except at the burst of the internet bubble (i.e., 2000-2002). In particular, the CCC model gives very erratic conditional betas during this period, while conditional market betas of the DCC and CHAR models are much more realistic.

Next, we compute one-step-ahead forecasts $\beta_{k, M K T, t+1 \mid t}$, $\beta_{k, S M B, t+1 \mid t}$ and $\beta_{k, H M L, t+1 \mid t}$, for the four models and the 12 industry portfolios. The sample-size has been chosen so that the first forecast corresponds to the first working day of year 2010 (i.e., 4th of January 2010), which is highlighted by a vertical line in Figs. 5 and 6 . The total number of forecasts is 1678 . For the sake of illustration, the predicted betas of the first portfolio (BusEq) are plotted in Fig. 8 . Recall that the models are reevaluated every 3 steps, reason why the forecasts of the model with constant betas (i.e., C-CHAR) are slowly changing over-time. Several comments are in order.
First, we observe huge differences between the forecasts produced by the four models. Focusing on the conditional market beta (i.e., the top graph in Fig. 8), the CHAR forecasts (red continuous line) are much smoother than those of the CCC-DCB and DCC-DCB models (green and purple dotted lines, respectively). The same comment applies to the other industry portfolios (graphs are not reported to save space but are available upon request).

Second, the CHAR model gives a tradeoff between the very smooth behavior of the C-CHAR model and the shaky behavior of the two DCB models. Interestingly, forecasts of the conditional HML beta of the DCC model deviate much from the forecasts of the other models between March-2013 and August-2013. Indeed, the DCC predicts an almost zero beta while the other 3 models keep predicting conditional betas close to their sample mean. In a risk management perspective, if the objective is to offset the impact of the HML factor onto the Buseq portfolio, the message given by the DCC during this period is to cut the hedging component of the portfolio, while the message of the other three models is to continue with the same risk management policy.

Finally, for each model, the predicted conditional betas are used to build a hedging portfolio used to immunize industry portfolios against all factors. The returns of this portfolio are easily obtained using the forecasted conditional betas, i.e.,

$$
\begin{aligned}
Z_{k, t+1 \mid t}= & \beta_{k, M K T, t+1 \mid t} M K T_{t+1}+\beta_{k, S M B, t+1 \mid t} S M B_{t+1} \\
& +\beta_{k, H M L, t+1 \mid t} H M L_{t+1},
\end{aligned}
$$

where $M K T_{t+1}, S M B_{t+1}$ and $H M L_{t+1}$ are the realized (nondemeaned) log-returns of the three factors. Economically, this hedging portfolio corresponds to the portfolio invested in the risk factors that optimally tracks the corresponding industry portfolio return. The term hedging means that we can short sell this portfolio to cover the main risks of a given portfolio.

For each of the 12 industry portfolios and the four different hedging portfolios, we compute the realized tracking error as follows:

$T E_{k, t+1}=r_{k, t+1}-Z_{k, t+1 \mid t}$

and seek for the model with the smallest sample mean square error (MSE) or mean absolute deviation (MAD) over the 1678 values of the tracking error by means of the Model Confidence Set of Hansen et al. (2011). A similar approach has been used by Hansen et al. (2014) in the context of Realized Beta GARCH models and more recently by Boudt et al. (2017) on the one-step-ahead forecasts of the CholCov estimator. The Fama-French three factor model, 

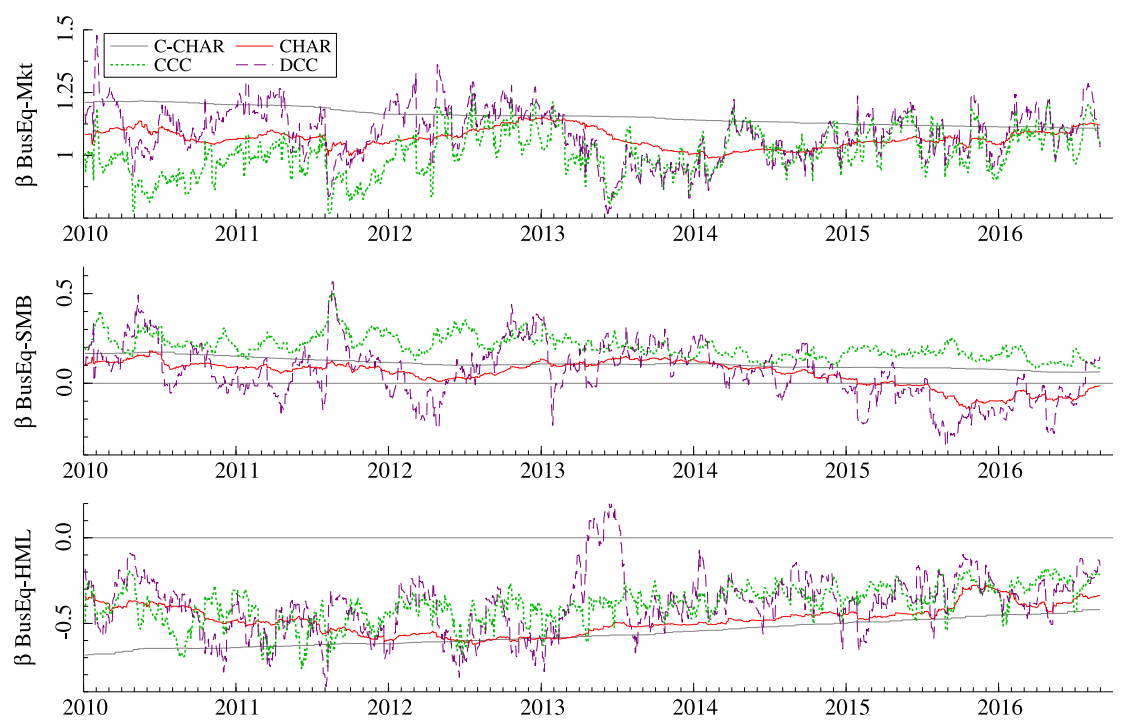

Fig. 8. One-step ahead forecasts of the conditional betas of BusEq for the 4 competing models.

Table 4

Robust (HAC) $t$-statistic of the regression of the tracking errors on a constant.

\begin{tabular}{lrrrrrrrrr}
\hline & \multicolumn{1}{l}{ C- } & CHAR & \multicolumn{1}{l}{ CCC } & \multicolumn{1}{l}{ DCC } & & \multicolumn{1}{l}{ C- } \\
CHAR & & & & & CHAR & CCC & DCC \\
\hline BusEq & -1.025 & -0.442 & 0.133 & -0.889 & Money & -0.699 & -0.648 & -0.837 & -0.541 \\
Chems & 0.683 & 0.150 & 1.187 & 0.252 & NoDur & $\mathbf{2 . 7 3 1}$ & $\mathbf{2 . 1 8 0}$ & $\mathbf{2 . 9 6 1}$ & $\mathbf{2 . 3 4 3}$ \\
Durbl & -0.059 & -0.402 & -0.123 & -0.720 & Other & -0.193 & -0.436 & -0.394 & -0.415 \\
Enrgy & -1.191 & -1.865 & -0.528 & -1.665 & Shops & 1.746 & 1.947 & $\mathbf{2 . 4 4 7}$ & 1.768 \\
Hlth & 1.664 & 1.123 & 1.777 & 1.325 & Telcm & 1.392 & 1.604 & $\mathbf{2 . 2 4 7}$ & 1.858 \\
Manuf & -0.101 & -0.269 & 0.064 & -0.197 & Utils & 1.053 & 0.858 & 1.549 & 1.010 \\
\hline
\end{tabular}

Note: Robust (HAC) $t$-statistics for the null hypothesis that the coefficient in the regression of $T E_{k, t+1}$ on a constant is zero. Values in bold are greater (in absolute value) than the critical value at the $5 \%$ significance level.

predicts that these factors are sufficient to price industry portfolios and therefore the tracking error is expected to have a zero mean (or zero alpha). Before reporting the results of the MCS test, we test the validity of this assumption for the four competing model by regressing $T E_{k, t+1}$ on a constant (on the whole out-of-sample period). Robust (HAC) t-statistics for the null hypothesis that this constant is zero are reported in Table 4. Results are in favor of this assumption in all but one case for the C-CHAR, CHAR and DCC-DCB models and for 9 cases for the CCC-DCB. The only industry portfolio for which this assumption is violated at the $5 \%$ significance level is NoDur

Table 5 contains the results of the MCS test with a MSE loss function, with a significance level of $5 \%$, and 10,000 bootstrap samples (with a block length of 5 observations). Models highlighted with the symbol $\checkmark$ are contained in the model confidence set (or set of superior models). The CHAR tracking portfolios always belong to the set of superior models while the DCC-DCB appears in the MCS in only 3 cases. ${ }^{9}$ Interestingly, the C-CHAR is rejected in all but two cases from the MCS suggesting that the assumption of constant betas leads to inferior tracking portfolios. The CCC-DCB models are also rejected from the MCS in all cases despite the fact that although this model imposes the constancy of the correlations it allows the conditional betas to be time-varying. Very similar results are obtained (but not reported to save place) with a MAD loss function (the only difference is that the DCC model appears in the MCS in only 2 cases instead of 3 ). The main conclusion drawn from Table 5 is that the conditional beta forecasts produced

\footnotetext{
9 While summary statistics on the realized tracking errors are not reported in Table 5, it is worth to mention that the CHAR model has the smallest MSE (and MAD) in 11 cases.
}

by the CHAR model give the best tracking portfolios and that the DCC-DCB model is not statistically inferior in 3 cases when relying on a MSE loss function.

A natural question arises on whether, the conditional beta forecasts of the CHAR model lead to more or less transaction costs than the DCC-DCB model. To answer this question let $\Delta_{\beta_{k, M K T}}=$ $\sum_{t=2}^{1678}\left|\beta_{k, M K T, t+1 \mid t}-\beta_{k, M K T, t \mid t-1}\right|$ be the sum of the absolute value of the variations of the predicted conditional stock market betas of the $k$ th industry portfolio for a given model. A higher value of $\Delta_{\beta_{k, M K T}}$ translates naturally into more transaction costs. Column MKT in Table 6 corresponds to the ratio between the value of $\Delta_{\beta_{k, M K T}}$ obtained for the CHAR and the DCC-DCB models. Columns SMB and HML correspond to the same ratio but for the other two factors. A value close to 1 means that the two models deliver equally stable beta forecasts while a value smaller (resp. greater) than 1 means that the CHAR (resp. DCC-DCB) model delivers more stable beta forecasts and therefore less (resp. more) transaction costs. Interestingly, all ratios are well below 1 (i.e., between about 0.3 and 0.7 ) suggesting that the conditional beta forecasts of the CHAR model are much smoother than those of the DCC-DCB model and therefore the CHAR model induces much less transaction costs.

\section{Conclusion}

This paper introduces a new model to estimate time series regressions with time-varying coefficients, called conditional betas in some financial applications. The CHAR model (for CholeskyGARCH model), is inspired by the model of Pourahmadi (1999), originally proposed in a longitudinal data framework. Unlike conditional correlations, conditional betas need not be constrained, except for the stationarity condition, which makes this approach 
Table 5

Results of the MCS test.

\begin{tabular}{|c|c|c|c|c|c|c|c|c|c|}
\hline & C-CHAR & CHAR & $\mathrm{CCC}$ & DCC & & C-CHAR & CHAR & CCC & DCC \\
\hline BusEq & & $\checkmark$ & & & Money & & $\checkmark$ & & $\checkmark$ \\
\hline Chems & & $\checkmark$ & & & NoDur & & $\checkmark$ & & \\
\hline Durbl & & $\checkmark$ & & & Other & $\checkmark$ & $\checkmark$ & & \\
\hline Enrgy & & $\checkmark$ & & $\checkmark$ & Shops & & $\checkmark$ & & \\
\hline Hlth & & $\checkmark$ & & $\checkmark$ & Telcm & $\checkmark$ & $\checkmark$ & & \\
\hline Manuf & & $\checkmark$ & & & Utils & & $\checkmark$ & & \\
\hline
\end{tabular}

The table shows the models included in the Model Confidence Set in the beta hedging exercise. Models highlighted with the symbol $\checkmark$ are contained in the model confidence set using a MSE loss function. The significance level for the MCS is set to $5 \%$, and 10,000 bootstrap samples (with a block length of 5 observations). The tracking error is defined as $T E_{k, t+1}=r_{k, t+1}-r_{0, t+1}-Z_{k, t+1 \mid t}$, where $Z_{k, t+1 \mid t}=\beta_{k, M K T, t+1 \mid t} M K T_{t+1}+\beta_{k, S M B, t+1 \mid t} S M B_{t+1}+\beta_{k, H M L, t+1 \mid t} H M L_{t+1}$.

Table 6

Transaction costs.

\begin{tabular}{llllllll}
\hline & MKT & SMB & HML & & MKT & SMB & HML \\
\hline BusEq & 0.356 & 0.380 & 0.341 & Money & 0.390 & 0.397 & 0.366 \\
Chems & 0.310 & 0.263 & 0.376 & NoDur & 0.414 & 0.383 & 0.296 \\
Durbl & 0.419 & 0.464 & 0.693 & Other & 0.273 & 0.343 & 0.335 \\
Enrgy & 0.373 & 0.337 & 0.456 & Shops & 0.344 & 0.297 & 0.395 \\
Hlth & 0.461 & 0.667 & 0.397 & Telcm & 0.334 & 0.414 & 0.640 \\
Manuf & 0.442 & 0.402 & 0.430 & Utils & 0.465 & 0.408 & 0.431
\end{tabular}

Note: Let $\Delta_{\beta_{k, j}}=\sum_{t=2}^{1,678}\left|\beta_{k, j, t+1 \mid t}-\beta_{k, j, t \mid t-1}\right|$ be the sum of the absolute value of the variations of the predicted conditional betas between the $k$ th industry portfolio and the $j$ th (where $j=$ MKT, SMB and HML) factor for a given model. For each column, the figures correspond to the ratio between the value of $\Delta_{\beta_{k, j}}$ obtained for the CHAR and the DCC-DCB models.

very appealing. Our model is flexible enough to directly specify the dynamics of conditional betas and impose the constancy of some of these coefficients. We study several specifications of the conditional beta dynamics and derive some stationarity and invertibility conditions. We also prove consistency and asymptotic normality of the Full and Equation-by-Equation QML estimators. The finite sample behavior of these two estimators is also numerically investigated by means of a Monte Carlo simulation.

We illustrate the usefulness of the CHAR model in a portfolio and risk management exercise. We follow Patton and Verardo (2012) and build hedged portfolios to offset some unwanted exposures to the three factors introduced in an asset pricing context by Fama and French $(1992,2004)$. We use four different models to forecast conditional betas, build tracking portfolios and compute tracking errors between forecasted and realized portfolio returns. We find that the CHAR model with time-varying betas is the one tracking the best the 12 US industry portfolios. Indeed, this model outperforms the three competing models and delivers much smoother conditional beta forecasts, which translates into smaller transaction costs.

We limit our investigations to dynamic specifications only involving past shocks and past conditional betas. Adding exogenous explanatory variables like in Patton and Verardo (2012) would allow the identification of variables influencing betas over time. As this would complicate the derivation of its statistical properties, we leave this for future work.

\section{Proofs}

Proof of Theorem 3.1. First note that the top Lyapunov $\gamma_{0}$ is well defined in $[-\infty, \infty)$ because $E \log ^{+}\left\|\boldsymbol{H}_{1}\right\|<\infty$. Using Bougerol and Picard (1992a), it is shown in Francq and Zakoïan (2012) that (2.7) admits a strictly stationary (and non anticipative) solution if and only if $\gamma_{0}<0$. Assume $\gamma_{0}<0$. The stationary and non anticipative solution is unique and ergodic, and is given by the $(2 q+1)$ th component of

$\boldsymbol{z}_{t}=\boldsymbol{h}_{t}+\sum_{k=1}^{\infty}\left(\prod_{i=0}^{k-1} \boldsymbol{H}_{t-i}\right) \boldsymbol{h}_{t-k}$
The process $\left(\boldsymbol{v}_{t}^{\prime}, \mathbf{g}_{t}^{\prime}\right)^{\prime}$ is then stationary and ergodic. By the ergodic theorem, the process $\left(\boldsymbol{c}_{0 t}\right)_{t}$, where $\boldsymbol{c}_{0 t}=\boldsymbol{c}_{0}\left(\boldsymbol{v}_{t}, \ldots, \boldsymbol{v}_{t-r+1}\right.$, $\left.\mathbf{g}_{t}, \ldots, \mathbf{g}_{t-r+1}\right)$, is also stationary and ergodic. Under the condition (ii), a strictly stationary non anticipative and ergodic solution to (2.8) ( or (2.9)) is defined by $\boldsymbol{\ell}_{t}$ (or $\boldsymbol{\beta}_{t}$ ) equal to

$$
\left\{\boldsymbol{I}_{m_{0}}-\sum_{i=1}^{s} \boldsymbol{c}_{0 i} B^{i}\right\}^{-1} \boldsymbol{c}_{0, t-1}=\boldsymbol{c}_{0, t-1}+\sum_{k=1}^{\infty} \Pi_{k} \boldsymbol{c}_{0, t-k-1},
$$

where $B$ denotes the Backward operator. The solution of the CHAR model is then defined by $\boldsymbol{\epsilon}_{t}=\boldsymbol{L}_{t} \boldsymbol{v}_{t}$.

Lemma 2.3 in Berkes et al. (2003) (see also Corollary 2.3 in (Francq and Zakoïan, 2010) shows that the strict stationarity condition $(i)$ implies the existence of $s_{0}>0$ such that $E\left\|\boldsymbol{z}_{1}\right\|^{s_{0}}<\infty$. The condition (iii) then entails $E\left\|\boldsymbol{c}_{01}\right\|^{2 s_{0}}<\infty$. Under the condition (ii), we have $\left\|\Pi_{k}\right\| \leq K \rho^{k}$ for some $K>0$ and $\rho \in[0,1)$. We thus have $E\left\|\ell_{1}\right\|^{s_{0}}<\infty$ for some $s_{0}>0$. By the Hölder inequality, we conclude that $\left\|\boldsymbol{\epsilon}_{t}\right\|$ and $\left\|\boldsymbol{\Sigma}_{t}\right\|$ admit a small order moment.

Without loss of generality, we now take the matrix norm defined by $\|\boldsymbol{M}\|=\sum_{i, j}\left|m_{i, j}\right|$ for any matrix $\boldsymbol{M}$ whose generic element is $m_{i, j}$. Noting that $\|\boldsymbol{A}\|\|\boldsymbol{B}\|=\|\boldsymbol{A} \otimes \boldsymbol{B}\|$ and using elementary properties of the Kronecker product, we obtain

$E\left\|\prod_{i=0}^{k-1} \boldsymbol{H}_{t-i} \boldsymbol{h}_{t-k}\right\|^{m}=E\left\|\prod_{i=0}^{k-1} \boldsymbol{H}_{t-i}^{\otimes m_{1}} \boldsymbol{h}_{t-k}^{\otimes m_{1}}\right\|=\left\|\left(E \boldsymbol{H}_{1}^{\otimes m_{1}}\right)^{k} E \boldsymbol{h}_{1}^{\otimes m_{1}}\right\|$.

Therefore, under the conditions (3.1), the $L^{m_{1}}$-norm of the $k$ th term of the $\operatorname{sum}(8.1)$ is bounded by $K \rho^{k}$, and thus $E\left\|\boldsymbol{z}_{t}\right\|^{m_{1}}<\infty$, which entails $E\left\|\boldsymbol{v}_{t}\right\|^{2 m_{1}}<\infty$ and $E\left\|\boldsymbol{g}_{t}\right\|^{m_{1}}<\infty$. Under the condition iii), we then have $E\left\|\boldsymbol{c}_{0 t}\right\|^{2 m_{1}}<\infty$, and thus $E\left\|\boldsymbol{\epsilon}_{1}\right\|^{2 m_{1}}<\infty$ under ii).

Proof of Corollary 3.1. We write the proof for Model (2.10)(2.11), the proof for Model (2.12)-(2.13) being identical. Under the Condition 1) it is well known that $g_{1 t}$ is well defined and that $v_{1 t}=$ $\sqrt{g_{1 t}} \eta_{1 t}$ is a stationary GJR process. Assume that there exist stationary, non anticipative and ergodic processes $\left(v_{1, t}, \ldots, v_{i_{0}-1, t}\right)$ and $\left(g_{1, t}, \ldots, g_{i_{0}-1, t}\right)$ satisfying $(2.10)$ for $i<i_{0}$ then, by the ergodic theorem, the process

$z_{i_{0}, t}=\omega_{0 i_{0}}+\gamma_{0 i_{0}+}\left(\epsilon_{1, t-1}^{+}\right)^{2}+\gamma_{0 i_{0}-}\left(\epsilon_{1, t-1}^{-}\right)^{2}+\sum_{k=2}^{i_{0}-1} \alpha_{0 i_{0}}^{(k)} v_{k, t-1}^{2}$

and, under 3), the processes

$$
\begin{aligned}
\beta_{i j} & =\sum_{\ell=0}^{\infty} c_{0 i j}^{\ell} u_{i j, t-1-\ell}, \\
u_{i j, t} & =\varpi_{0 i j}+\varsigma_{0 i j+} \epsilon_{1, t-1}^{+}+\varsigma_{0 i j-} \epsilon_{1, t-1}^{-}+\sum_{k=2}^{i} \tau_{0 i j}^{(k)} v_{k, t-1},
\end{aligned}
$$

for $i<i_{0}$ and $(i, j) \in \boldsymbol{T}_{m}$ inherit the property of being stationary, non anticipative and ergodic. By Cauchy's rule, under Condition 2), 
the process

$g_{i_{0}, t}=z_{i_{0}, t}+\sum_{\ell=1}^{\infty} z_{i_{0}, t-\ell} \prod_{k=1}^{\ell}\left\{\alpha_{0 i_{0}}^{\left(i_{0}\right)} \eta_{i_{0}, t-k}^{2}+b_{0 i_{0}}\right\}$

is also stationary, non anticipative and ergodic. The existence of the strictly stationary solution is thus obtained by mathematical induction on $i_{0}$. The existence of the moments is shown as in Theorem 3.1.

Proof of Theorem 4.1. By compactness of $\Theta$, using the arguments of Wald (1949), to establish the strong consistency it suffices to show that for all $\vartheta \neq \vartheta_{0}$, there exists a neighborhood $V(\vartheta)$ of $\vartheta$ such that

$\liminf _{n \rightarrow \infty} \inf _{\boldsymbol{\vartheta}^{*} \in V(\boldsymbol{\vartheta})} \widetilde{O}_{n}\left(\boldsymbol{\vartheta}^{*}\right)>\lim _{n \rightarrow \infty} \widetilde{O}_{n}\left(\boldsymbol{\vartheta}_{0}\right), \quad$ a.s.

We first show that $\widetilde{O}_{n}$ can be replaced by $O_{n}$, where

$O_{n}(\vartheta)=\frac{1}{n} \sum_{t=1}^{n} q_{t}(\vartheta)$

$q_{t}(\boldsymbol{\vartheta})=\boldsymbol{\epsilon}_{t}^{\prime} \boldsymbol{B}_{t}^{\prime}(\boldsymbol{\vartheta}) \boldsymbol{G}_{t}^{-1}(\boldsymbol{\vartheta}) \boldsymbol{B}_{t}(\boldsymbol{\vartheta}) \boldsymbol{\epsilon}_{t}+\sum_{i=1}^{m} \log g_{i t}(\boldsymbol{\vartheta})$.

Omitting the subscript “( $\vartheta)$ " in the notations, we have

$$
\begin{aligned}
\sup _{\vartheta \in \Theta}\left|O_{n}-\widetilde{O}_{n}\right| & \leq \frac{1}{n} \sum_{t=1}^{n} \sup _{\vartheta \in \Theta}\left|\boldsymbol{\epsilon}_{t}^{\prime}\left(\boldsymbol{B}_{t} \boldsymbol{G}_{t}^{-1} \boldsymbol{B}_{t}^{\prime}-\widetilde{\boldsymbol{B}}_{t} \widetilde{\boldsymbol{G}}_{t}^{-1} \widetilde{\boldsymbol{B}}_{t}\right) \boldsymbol{\epsilon}_{t}\right| \\
& +\frac{1}{n} \sum_{t=1}^{n} \sum_{i=1}^{m} \sup _{\vartheta \in \Theta}\left|\log g_{i t}-\log \widetilde{g}_{i t}\right| .
\end{aligned}
$$

Note that we have the decomposition $\boldsymbol{B}_{t} \boldsymbol{G}_{t}^{-1} \boldsymbol{B}_{t}^{\prime}-\widetilde{\boldsymbol{B}}_{t} \widetilde{\boldsymbol{G}}_{t}^{-1} \widetilde{\boldsymbol{B}}_{t}=A_{t}+$ $B_{t}+C_{t}$, with

$A_{t}=\left(\boldsymbol{B}_{t}-\widetilde{\boldsymbol{B}}_{t}\right) \boldsymbol{G}_{t}^{-1} \boldsymbol{B}_{t}^{\prime}, \quad B_{t}=\widetilde{\boldsymbol{B}}_{t} \boldsymbol{G}_{t}^{-1}\left(\widetilde{\boldsymbol{G}}_{t}-\boldsymbol{G}_{t}\right) \widetilde{\boldsymbol{G}}_{t}^{-1} \boldsymbol{B}_{t}$,

$C_{t}=\widetilde{\boldsymbol{B}}_{t} \widetilde{\boldsymbol{G}}_{t}^{-1}\left(\boldsymbol{B}_{t}-\widetilde{\boldsymbol{B}}_{t}\right)$.

In the sequel, we take the spectral norm as matrix norm and the Euclidean norm as vector norm. Using A1 -A2, we obtain

$$
\begin{aligned}
& \frac{1}{n} \sum_{t=1}^{n} \sup _{\vartheta \in \Theta}\left|\boldsymbol{\epsilon}_{t}^{\prime} A_{t} \boldsymbol{\epsilon}_{t}\right|=\frac{1}{n} \sum_{t=1}^{n} \sup _{\vartheta \in \Theta}\left|\operatorname{Tr}\left\{\left(\boldsymbol{B}_{t}-\widetilde{\boldsymbol{B}}_{t}\right) \boldsymbol{G}_{t}^{-1} \boldsymbol{B}_{t}^{\prime} \boldsymbol{\epsilon}_{t} \boldsymbol{\epsilon}_{t}^{\prime}\right\}\right| \\
\leq & K \frac{1}{n} \sum_{t=1}^{n} \sup _{\vartheta \in \Theta}\left\|\boldsymbol{\beta}_{t}-\widetilde{\boldsymbol{\beta}}_{t}\right\|\left\|\boldsymbol{g}_{t}^{-1}\right\|\left\|\boldsymbol{\beta}_{t}\right\|\left\|\boldsymbol{\epsilon}_{t} \boldsymbol{\epsilon}_{t}^{\prime}\right\| \\
\leq & K \frac{1}{n} \sum_{t=1}^{n} \rho_{t}\left\|\boldsymbol{\epsilon}_{t} \boldsymbol{\epsilon}_{t}^{\prime}\right\| \sup _{\vartheta \in \Theta}\left\|\boldsymbol{\beta}_{t}\right\| .
\end{aligned}
$$

Moreover $\sum_{t=1}^{\infty} \rho_{t}\left\|\boldsymbol{\epsilon}_{t} \boldsymbol{\epsilon}_{t}^{\prime}\right\| \sup _{\boldsymbol{\vartheta} \in \Theta}\left\|\boldsymbol{\beta}_{t}\right\|$ is finite a.s. since

$$
\begin{aligned}
& E\left(\sum_{t=1}^{\infty} \rho_{t}\left\|\boldsymbol{\epsilon}_{t}^{\prime} \boldsymbol{\epsilon}_{t}\right\| \sup _{\boldsymbol{\vartheta} \in \Theta}\left\|\boldsymbol{\beta}_{t}\right\|\right)^{s_{0}} \\
& \quad \leq\left\{E\left\|\boldsymbol{\epsilon}_{1}\right\|^{6 s_{0}}\right\}^{1 / 3}\left\{E\left(\sup _{\boldsymbol{\vartheta} \in \Theta}\left\|\boldsymbol{\beta}_{1}\right\|\right)^{3 s_{0}}\right\}^{1 / 3} \sum_{t=1}^{\infty}\left\{E \rho_{t}^{3 s_{0}}\right\}^{1 / 3}<\infty
\end{aligned}
$$

for $s_{0}$ small enough, by $\mathbf{A} \mathbf{2}$ and $\mathbf{A} 3$. The same bounds are obtained when $A_{t}$ is replaced by $B_{t}$ or $C_{t}$. Therefore the first term of the right-hand side of the inequality (8.3) is of order $O\left(n^{-1}\right)$ a.s. Now, consider the second term. The elementary inequality $\log (x) \leq x+1$ and $\mathbf{A 1} \mathbf{- A 2}$ entail

$\sup _{\vartheta \in \Theta}\left|\log g_{i t}-\log \widetilde{g}_{i t}\right|=\sup _{\vartheta \in \Theta}\left|\log \left(1+\frac{\widetilde{g}_{i t}-g_{i t}}{g_{i t}}\right)\right| \leq K \rho_{t}$, noting that $g_{i t}^{-1} \leq\left\|g_{t}^{-1}\right\|$. We thus have shown that

$\sup _{\boldsymbol{\vartheta} \in \Theta}\left|O_{n}(\boldsymbol{\vartheta})-\widetilde{O}_{n}(\boldsymbol{\vartheta})\right|=O\left(n^{-1}\right) \quad$ a.s.

We now show that $E q_{t}(\boldsymbol{\vartheta})$ is well defined in $\mathbb{R} \cup\{+\infty\}$ for all $\vartheta$, and in $\mathbb{R}$ for $\vartheta=\vartheta_{0}$. By $\mathbf{A 1}$ we have

$E q_{t}^{-}(\vartheta) \leq \sum_{i=1}^{m} E \log ^{-} g_{i t}(\vartheta)<\infty$

At $\boldsymbol{\vartheta}_{0}$, Jensen's inequality and $\mathbf{A} \mathbf{3}$ entail

$E q_{t}\left(\boldsymbol{\vartheta}_{0}\right)=E \boldsymbol{\eta}_{t}^{\prime} \boldsymbol{\eta}_{t}+\sum_{i=1}^{m} \frac{1}{s} E \log g_{i t}^{s}\left(\boldsymbol{\vartheta}_{0}\right)<\infty$.

The previous results and the ergodic theorem then entail that $\lim _{n \rightarrow \infty} \widetilde{O}_{n}\left(\boldsymbol{\vartheta}_{0}\right)=\lim _{n \rightarrow \infty} O_{n}\left(\boldsymbol{\vartheta}_{0}\right)=E q_{t}\left(\boldsymbol{\vartheta}_{0}\right)$.

Similarly, (8.4) and the ergodic theorem applied to the stationary process $\left(X_{t}\right)$ with $X_{t}=\inf _{\vartheta^{*} \in V_{m}(\vartheta)} q_{t}\left(\vartheta^{*}\right)$ show that

$$
\begin{aligned}
\liminf _{n \rightarrow \infty} \inf _{\boldsymbol{\vartheta}^{*} \in V_{m}(\boldsymbol{\vartheta})} \widetilde{O}_{n}\left(\boldsymbol{\vartheta}^{*}\right) & \geq \lim _{n \rightarrow \infty} \frac{1}{n} \sum_{t=1}^{n} \inf _{\boldsymbol{\vartheta}^{*} \in V_{m}(\boldsymbol{\vartheta})} q_{t}\left(\boldsymbol{\vartheta}^{*}\right) \\
& =E \inf _{\boldsymbol{\vartheta}^{*} \in V_{m}(\boldsymbol{\vartheta})} q_{t}\left(\boldsymbol{\vartheta}^{*}\right),
\end{aligned}
$$

where $V_{m}(\boldsymbol{\vartheta})$ denotes the ball of center $\vartheta$ and radius $1 / \mathrm{m}$. If $E\left|q_{t}(\boldsymbol{\vartheta})\right|<\infty$, by Fatou's lemma and A5, for any $\varepsilon>0$ there exists $m$ sufficiently large such that

$$
E \inf _{\vartheta^{*} \in V_{m}(\vartheta)} q_{t}\left(\vartheta^{*}\right)>E q_{t}(\vartheta)-\varepsilon .
$$

If $E q_{t}^{+}(\vartheta)=\infty$, then the left-hand side of the previous inequality can be made arbitrarily large.

To show (8.2), it thus remains to show that $E q_{t}(\vartheta)$ is minimized at $\boldsymbol{\vartheta}_{0}$. Without loss of generality, assume that $\operatorname{Eq}_{t}^{+}(\boldsymbol{\vartheta})<\infty$. Let $\lambda_{i, t}$ be the eigenvalues of $\boldsymbol{\Sigma}_{t}\left(\boldsymbol{\vartheta}_{0}\right) \boldsymbol{\Sigma}_{t}^{-1}(\boldsymbol{\vartheta})$, which are positive. We have

$$
\begin{aligned}
E q_{t}(\boldsymbol{\vartheta})-E q_{t}\left(\boldsymbol{\vartheta}_{0}\right)= & E \log \left\{\left|\boldsymbol{\Sigma}_{t}(\boldsymbol{\vartheta}) \boldsymbol{\Sigma}_{t}^{-1}\left(\boldsymbol{\vartheta}_{0}\right)\right|\right\} \\
& +E\left(\operatorname{Tr}\left\{\left[\boldsymbol{\Sigma}_{t}\left(\boldsymbol{\vartheta}_{0}\right) \Sigma_{t}^{-1}(\boldsymbol{\vartheta})-\boldsymbol{I}_{m}\right]\right\}\right) \\
= & E\left\{\sum_{i=1}^{m}\left(\lambda_{i t}-1-\log \lambda_{i t}\right)\right\} \geq 0,
\end{aligned}
$$

where the inequality is strict unless if $\lambda_{i t}=1$ a.s. for all $i$, that is iff $\boldsymbol{\Sigma}_{t}(\boldsymbol{\vartheta})=\boldsymbol{\Sigma}_{t}\left(\boldsymbol{\vartheta}_{0}\right)$ a.s., which is equivalent to $\boldsymbol{\vartheta}=\boldsymbol{\vartheta}_{0}$ under A4. The consistency follows.

Elementary matrix derivative computations yield

$$
\begin{gathered}
\frac{\partial}{\partial \vartheta_{i}} q_{t}\left(\boldsymbol{\vartheta}_{0}\right)=\operatorname{Tr}\left\{\left(\boldsymbol{I}_{m}-\boldsymbol{\eta}_{t} \boldsymbol{\eta}_{t}^{\prime}\right) \boldsymbol{\Sigma}_{t}^{-1 / 2}\left(\boldsymbol{\vartheta}_{0}\right) \frac{\partial \boldsymbol{\Sigma}_{t}\left(\boldsymbol{\vartheta}_{0}\right)}{\vartheta_{i}} \boldsymbol{\Sigma}_{t}^{-1 / 2^{\prime}}\left(\boldsymbol{\vartheta}_{0}\right)\right\} \\
=\frac{\partial \operatorname{vec}^{\prime} \boldsymbol{\Sigma}_{t}\left(\boldsymbol{\vartheta}_{0}\right)}{\partial \vartheta_{i}}\left\{\boldsymbol{\Sigma}_{t}^{-1 / 2^{\prime}}\left(\boldsymbol{\vartheta}_{0}\right) \otimes \boldsymbol{\Sigma}_{t}^{-1 / 2^{\prime}}\left(\boldsymbol{\vartheta}_{0}\right)\right\} \operatorname{vec}\left(\boldsymbol{I}_{m}-\boldsymbol{\eta}_{t} \boldsymbol{\eta}_{t}^{\prime}\right),
\end{gathered}
$$

where "Tr", "vec" and “ $\otimes$ " of denote respectively the trace, vec and Kronecker operators. We also have

$$
\frac{\partial^{2}}{\partial \vartheta_{i} \partial \vartheta_{j}} q_{t}(\vartheta)=\sum_{i=1}^{5} c_{i}
$$

with

$$
\begin{aligned}
c_{1} & =\boldsymbol{\epsilon}_{t}^{\prime} \Sigma_{t}^{-1}(\boldsymbol{\vartheta}) \frac{\partial \boldsymbol{\Sigma}_{t}(\boldsymbol{\vartheta})}{\partial \vartheta_{i}} \Sigma_{t}^{-1}(\boldsymbol{\vartheta}) \frac{\partial \boldsymbol{\Sigma}_{t}(\boldsymbol{\vartheta})}{\partial \vartheta_{j}} \Sigma_{t}^{-1}(\boldsymbol{\vartheta}) \boldsymbol{\epsilon}_{t}, \\
c_{2} & =\boldsymbol{\epsilon}_{t}^{\prime} \boldsymbol{\Sigma}_{t}^{-1}(\boldsymbol{\vartheta}) \frac{\partial \boldsymbol{\Sigma}_{t}(\boldsymbol{\vartheta})}{\partial \vartheta_{j}} \Sigma_{t}^{-1}(\boldsymbol{\vartheta}) \frac{\partial \boldsymbol{\Sigma}_{t}(\boldsymbol{\vartheta})}{\partial \vartheta_{i}} \Sigma_{t}^{-1}(\boldsymbol{\vartheta}) \boldsymbol{\epsilon}_{t} \\
c_{3} & =-\boldsymbol{\epsilon}_{t}^{\prime} \boldsymbol{\Sigma}_{t}^{-1}(\boldsymbol{\vartheta}) \frac{\partial^{2} \boldsymbol{\Sigma}_{t}(\boldsymbol{\vartheta})}{\partial \vartheta_{i} \partial \vartheta_{j}} \Sigma_{t}^{-1}(\boldsymbol{\vartheta}) \boldsymbol{\epsilon}_{t}
\end{aligned}
$$




$$
\begin{aligned}
c_{4} & =-\operatorname{Tr}\left(\frac{\partial \boldsymbol{\Sigma}_{t}(\boldsymbol{\vartheta})}{\partial \vartheta_{i}} \boldsymbol{\Sigma}_{t}^{-1}(\boldsymbol{\vartheta}) \frac{\partial \boldsymbol{\Sigma}_{t}(\boldsymbol{\vartheta})}{\partial \vartheta_{j}} \boldsymbol{\Sigma}_{t}^{-1}(\boldsymbol{\vartheta})\right) \\
c_{5} & =\operatorname{Tr}\left(\boldsymbol{\Sigma}_{t}^{-1}(\boldsymbol{\vartheta}) \frac{\partial^{2} \boldsymbol{\Sigma}_{t}(\boldsymbol{\vartheta})}{\partial \vartheta_{i} \partial \vartheta_{j}}\right) .
\end{aligned}
$$

We thus have

$$
\begin{aligned}
& E\left\{\frac{\partial^{2}}{\partial \vartheta_{i} \partial \vartheta_{j}} q_{t}\left(\boldsymbol{\vartheta}_{0}\right)\right\} \\
& \quad=\operatorname{Tr}\left(\boldsymbol{\Sigma}_{t}^{-1 / 2}(\boldsymbol{\vartheta}) \frac{\partial \boldsymbol{\Sigma}_{t}(\boldsymbol{\vartheta})}{\partial \vartheta_{i}} \Sigma_{t}^{-1}(\boldsymbol{\vartheta}) \frac{\partial \boldsymbol{\Sigma}_{t}(\boldsymbol{\vartheta})}{\partial \vartheta_{j}} \Sigma_{t}^{-1 / 2^{\prime}}(\boldsymbol{\vartheta})\right) \\
& =\operatorname{vec}\left(\frac{\partial \boldsymbol{\Sigma}_{t}(\boldsymbol{\vartheta})}{\partial \vartheta_{i}}\right)^{\prime} \boldsymbol{\Sigma}_{t}^{-1}(\boldsymbol{\vartheta}) \otimes \boldsymbol{\Sigma}_{t}^{-1}(\boldsymbol{\vartheta}) \operatorname{vec}\left(\frac{\partial \boldsymbol{\Sigma}_{t}(\boldsymbol{\vartheta})}{\partial \vartheta_{i}}\right),
\end{aligned}
$$

using elementary properties of the vec and Kronecker operators

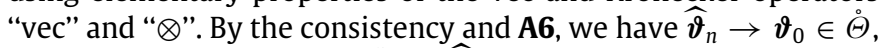
and thus almost surely $\partial \sum_{t=1}^{n} \widetilde{q}_{t}\left(\widehat{\vartheta}_{n}\right) / \partial \vartheta=\mathbf{0}$ for $n$ large enough. Taylor expansions and $\mathbf{A 7 - A 8}$ thus show that almost surely

$$
\begin{aligned}
\mathbf{0}= & \frac{1}{\sqrt{n}} \sum_{t=1}^{n} \frac{\partial q_{t}\left(\boldsymbol{\vartheta}_{0}\right)}{\partial \boldsymbol{\vartheta}} \\
& +\left(\frac{1}{n} \sum_{t=1}^{n} \frac{\partial^{2}}{\partial \vartheta_{i} \partial \vartheta_{j}} q_{t}\left(\boldsymbol{\vartheta}_{i j}^{*}\right)\right) \sqrt{n}\left(\widehat{\vartheta}_{n}-\vartheta_{0}\right)+o_{P}(1),
\end{aligned}
$$

where the $\boldsymbol{\vartheta}_{i j}^{*}$ 's are between $\widehat{\vartheta}_{n}$ and $\vartheta_{0}$ componentwise. To show that the previous matrix into brackets converges almost surely to $\boldsymbol{J}$ it suffices to use the ergodic theorem, the continuity of the derivatives, and to show that

$E \sup _{\vartheta \in V\left(\vartheta_{0}\right)}\left|\frac{\partial^{2} q_{t}(\vartheta)}{\partial \vartheta_{i} \partial \vartheta_{j}}\right|<\infty$

for some neighborhood $V\left(\boldsymbol{\vartheta}_{0}\right)$ of $\boldsymbol{\vartheta}_{0}$, which follows from A7 and A9.

If $\boldsymbol{J}$ was not invertible, there would exist some nonzero $\lambda \in \mathbb{R}^{d}$ such that $\lambda^{\prime} \boldsymbol{J} \lambda=0$. Since $\boldsymbol{\Sigma}_{t}^{-1}\left(\boldsymbol{\vartheta}_{0}\right) \otimes \boldsymbol{\Sigma}_{t}^{-1}\left(\boldsymbol{\vartheta}_{0}\right)$ is almost surely positive definite, this entails that $\boldsymbol{D}_{t} \lambda=\mathbf{0}$ with probability one, which is excluded by A11. The Bahadur linearization (4.2) easily follows.

It is clear from (8.13) below, $\mathbf{A 1 0}$ and already given arguments that $\left\{\frac{\partial}{\partial \vartheta} q_{t}\left(\boldsymbol{\vartheta}_{0}\right)\right\}_{t}$ is a square integrable martingale difference. The conclusion then follows from the central limit theorem in Billingsley (1961).

Proof of Theorem 4.2. First note that Corollary 3.1 and the condition $\left|b_{i}\right|<1$ uniformly in $\Theta$ entail that $\mathbf{A} 3$ is satisfied. Now recall that $\mathbf{B} 5$ entails (4.13). With the notation $\boldsymbol{v}_{t}(\boldsymbol{\varphi})=\boldsymbol{B}_{t}(\boldsymbol{\varphi}) \boldsymbol{\epsilon}_{t}$ and $\widetilde{\boldsymbol{v}}_{t}(\boldsymbol{\varphi})=\widetilde{\boldsymbol{B}}_{t}(\boldsymbol{\varphi}) \boldsymbol{\epsilon}_{t}$, we thus have

$$
\sup _{\boldsymbol{\varphi} \in \Theta_{\varphi}}\left\|\boldsymbol{v}_{t}(\boldsymbol{\varphi})-\widetilde{\boldsymbol{v}}_{t}(\boldsymbol{\varphi})\right\| \leq K \rho^{t}\left\|\boldsymbol{\epsilon}_{t}\right\| .
$$

Thanks to the condition $\left|b_{i}\right|<1$ in $\mathbf{B 3}$, one can then define $\mathbf{g}_{t}(\boldsymbol{\vartheta})=$ $\left(g_{1 t}\left(\boldsymbol{\vartheta}^{(+1)}\right), \ldots, g_{m t}\left(\boldsymbol{\vartheta}^{(+m)}\right)\right)^{\prime}$ by

$g_{i t}\left(\boldsymbol{\vartheta}^{(+i)}\right)=\sum_{j=0}^{\infty} b_{i}^{j}\left\{\omega_{i, t-j-1}+\sum_{k=2}^{i} \alpha_{i}^{(k)} v_{k, t-j-1}^{2}\left(\varphi^{(+k)}\right)\right\}$.

One can also define $q_{t}(\boldsymbol{\vartheta})=\sum_{i=1}^{m} q_{i t}\left(\boldsymbol{\vartheta}^{(+i)}\right)$, where $q_{i t}\left(\boldsymbol{\vartheta}^{(+i)}\right)$ is obtained by replacing $\widetilde{v}_{k t}\left(\varphi^{(+i)}\right)$ and $\widetilde{g}_{i t}\left(\boldsymbol{\vartheta}^{(+i)}\right)$ by $v_{k t}\left(\varphi^{(+i)}\right)$ and $g_{i t}\left(\boldsymbol{\vartheta}^{(+i)}\right)$ in $\widetilde{q}_{i t}\left(\boldsymbol{\vartheta}^{(+i)}\right)$.

Using (8.5) and the compactness of $\Theta$, we have

$$
\begin{aligned}
& \left|g_{i t}\left(\boldsymbol{\vartheta}^{(+i)}\right)-\tilde{g}_{i t}\left(\boldsymbol{\vartheta}^{(+i)}\right)\right| \\
= & \mid \sum_{k=2}^{i} \alpha_{i}^{(k)}\left\{v_{k, t-1}^{2}\left(\boldsymbol{\varphi}^{(+k)}\right)-\widetilde{v}_{k, t-1}^{2}\left(\boldsymbol{\varphi}^{(+k)}\right)\right\}
\end{aligned}
$$

$$
\begin{array}{r}
+b_{i}\left\{g_{i, t-1}\left(\boldsymbol{\vartheta}^{(+i)}\right)-\widetilde{g}_{i, t-1}\left(\boldsymbol{\vartheta}^{(+i)}\right)\right\} \mid \\
\leq K \rho^{t-1}\left\|\boldsymbol{\epsilon}_{t-1}\right\|\left(\left\|\boldsymbol{v}_{t-1}\right\|+\left\|\boldsymbol{\epsilon}_{t-1}\right\|\right) \\
+\rho\left|g_{i, t-1}\left(\boldsymbol{\vartheta}^{(+i)}\right)-\widetilde{g}_{i, t-1}\left(\boldsymbol{\vartheta}^{(+i)}\right)\right| .
\end{array}
$$

It follows that

$\sup _{\boldsymbol{\vartheta} \in \Theta}\left\|\boldsymbol{g}_{t}(\boldsymbol{\vartheta})-\tilde{\mathbf{g}}_{t}(\boldsymbol{\vartheta})\right\| \leq K \rho_{t}, \quad \rho_{t}=\rho^{t} \sum_{i=1}^{t-1}\left\|\boldsymbol{\epsilon}_{i}\right\|\left(\left\|\boldsymbol{v}_{i}\right\|+\left\|\boldsymbol{\epsilon}_{i}\right\|\right)$.

For $s_{1}<s_{0} / 2$ and $s_{0}<1$ satisfying $\mathbf{A} 3$, we have

$$
\begin{aligned}
& \sum_{t=1}^{\infty}\left\{E \rho_{t}^{s_{1}}\right\}^{1 / 3} \\
& \quad \leq \sum_{t=1}^{\infty}\left\{t \rho^{s_{1} t}\left(E\left\|\boldsymbol{\epsilon}_{1}\right\|^{2 s_{1}}+\sqrt{E\left\|\boldsymbol{\epsilon}_{1}\right\|^{2 s_{1}} E\left\|\boldsymbol{v}_{1}\right\|^{2 s_{1}}}\right)\right\}^{1 / 3}<\infty .
\end{aligned}
$$

It follows that $\mathbf{A} \mathbf{2}$ is satisfied. The positivity constraints in $\mathbf{B} 3$ entail that $\mathbf{A 1}$ holds true. We then obtain the Eq. (8.4) that shows the asymptotic irrelevance of the initial values. Using the ergodic theorem, it follows that $\widetilde{O}_{n}(\boldsymbol{\vartheta})$ converges almost surely to $E q_{t}(\boldsymbol{\vartheta})=$ $\sum_{i=1}^{m} E q_{i t}\left(\boldsymbol{\vartheta}^{(+i)}\right)$, which is well defined in $\mathbb{R} \cup\{+\infty\}$ because

$$
\inf _{\vartheta^{(+i)} \in \Theta^{(+i)}} g_{i t}\left(\vartheta^{(+i)}\right)>0
$$

from B3. Since $E\left\|\mathbf{g}_{1}\right\|^{s_{0}}<\infty$ for some $s_{0}>0$ by Corollary 3.1, we have $E \log g_{i t}\left(\boldsymbol{\vartheta}_{0}^{(+i)}\right)<\infty$, and thus $E\left|q_{i t}\left(\vartheta_{0}^{(+i)}\right)\right|<\infty$. When $E q_{i t}\left(\vartheta^{(+i)}\right)<\infty$, we have

$$
\begin{aligned}
E q_{i t}\left(\boldsymbol{\vartheta}^{(+i)}\right)-E q_{i t}\left(\boldsymbol{\vartheta}_{0}^{(+i)}\right)= & E\left\{\log \frac{g_{i t}\left(\boldsymbol{\vartheta}^{(+i)}\right)}{g_{i t}\left(\boldsymbol{\vartheta}_{0}^{(+i)}\right)}+\frac{g_{i t}\left(\boldsymbol{\vartheta}_{0}^{(+i)}\right)}{g_{i t}\left(\boldsymbol{\vartheta}^{(+i)}\right)}-1\right\} \\
& +E \frac{\left\{v_{i t}\left(\boldsymbol{\varphi}^{(+i)}\right)-v_{i t}\left(\boldsymbol{\varphi}_{0}^{(+i)}\right)\right\}^{2}}{g_{i t}\left(\boldsymbol{\vartheta}^{(+i)}\right)} \\
& +2 E \frac{v_{i t}\left(\boldsymbol{\varphi}_{0}^{(+i)}\right)\left\{v_{i t}\left(\boldsymbol{\varphi}^{(+i)}\right)-v_{i t}\left(\boldsymbol{\varphi}_{0}^{(+i)}\right)\right\}}{g_{i t}\left(\boldsymbol{\vartheta}^{(+i)}\right)}
\end{aligned}
$$

with the convention $\varphi^{(+2)}=\varphi^{(2)}$ and $v_{1 t}\left(\varphi^{(+1)}\right)=\epsilon_{1 t}$. The last expectation in (8.7) is equal to zero because, when $i>1$,

$v_{i t}\left(\varphi^{(+i)}\right)-v_{i t}\left(\varphi_{0}^{(+i)}\right)=-\sum_{j=1}^{i-1}\left\{\beta_{i j, t}\left(\varphi^{(+i)}\right)-\beta_{i j, t}\left(\varphi_{0}^{(+i)}\right)\right\} \epsilon_{j t}$

is orthogonal to $v_{i t}=v_{i t}\left(\varphi_{0}^{(+i)}\right)$. By the inequality $x-1 \geq \log x$ for $x>0$, it follows that $E q_{i t}\left(\boldsymbol{\vartheta}^{(+i)}\right) \geq E q_{i t}\left(\boldsymbol{\vartheta}_{0}^{(+i)}\right)$ with equality if and only if

$v_{i t}\left(\boldsymbol{\varphi}^{(+i)}\right)=v_{i t}\left(\boldsymbol{\varphi}_{0}^{(+i)}\right) \quad$ and $\quad g_{i t}\left(\boldsymbol{\vartheta}^{(+i)}\right)=g_{i t}\left(\boldsymbol{\vartheta}_{0}^{(+i)}\right) \quad$ a.s.

In view of (2.10) and (4.6), these equalities entail

$0=\omega_{0 i, t-1}-\omega_{i, t-1}+\sum_{k=2}^{i}\left(\alpha_{0 i}^{(k)}-\alpha_{i}^{(k)}\right) v_{k, t-1}^{2}+\left(b_{0 i}-b_{i}\right) g_{i, t-1}$.

We thus have

$\left(\alpha_{0 i}^{(i)}-\alpha_{i}^{(i)}\right) \eta_{i, t-1}^{2}=R_{t-1}^{(-i)}$,

where $R_{t-1}^{(-i)}$ is a random variable measurable with respect to the $\sigma$-field $\mathcal{F}_{t-1}^{(-i)}$ generated by $\mathcal{F}_{t-2}$ and $\left\{\eta_{j, t-1}, j \neq i\right\}$. It follows that the distribution of $\left(\alpha_{0 i}^{(i)}-\alpha_{i}^{(i)}\right) \eta_{i, t-1}^{2}$ conditionally to $\mathcal{F}_{t-1}^{(-i)}$ is degenerated. Since $\eta_{i, t-1}^{2}$ is independent of $\mathcal{F}_{t-2}$, the distribution 
of $\left(\alpha_{0 i}^{(i)}-\alpha_{i}^{(i)}\right) \eta_{i, t-1}^{2}$ conditionally to $\left\{\eta_{j, t-1}, j \neq i\right\}$ is degenerated. By the first part of B2, it follows that $\alpha_{i}^{(i)}=\alpha_{0 i}^{(i)}$. Repeating the argument, we show that $\alpha_{i}^{(k)}=\alpha_{0 i}^{(k)}$ for $k=2, \ldots, i$. Now, noting that

$$
\begin{aligned}
\omega_{0 i, t-1}-\omega_{i, t-1} & =\omega_{0 i}-\omega_{i}+\left(\gamma_{0 i+}-\gamma_{i+}\right) g_{1, t-1} \eta_{1, t-1}^{2} 1_{\left\{\eta_{1, t-1}>0\right\}} \\
& +\left(\gamma_{0 i-}-\gamma_{i-}\right) g_{1, t-1} \eta_{1, t-1}^{2} 1_{\left\{\eta_{1, t-1}<0\right\}}
\end{aligned}
$$

we obtain

$\left(\gamma_{0 i+}-\gamma_{i+}\right) \eta_{1, t-1}^{2} 1_{\left\{\eta_{1, t-1}>0\right\}}+\left(\gamma_{0 i-}-\gamma_{i-}\right) \eta_{1, t-1}^{2} 1_{\left\{\eta_{1, t-1}<0\right\}}=R_{t-2}$,

where $R_{t}$ is a random variable measurable with respect to $\mathcal{F}_{t}$. An equation of the form $a x^{2} 1_{\{x>0\}}+b=0$ cannot admit two positive solutions when $a \neq 0$. Therefore the last part of $\mathbf{B 2}$ entails that $\gamma_{0 i+}=\gamma_{i+}$ and $\gamma_{0 i_{-}}=\gamma_{i-}$. We then obtain

$0=\omega_{0 i}-\omega_{i}+\left(b_{0 i}-b_{i}\right) g_{i, t-1}$,

which entails $\omega_{0 i}=\omega_{i}$ and $b_{0 i}=b_{i}$, when $g_{i, t-1}$ is not constant with probability one (which is guaranteed by B4 and B2). We have shown that (8.9) implies $\boldsymbol{\theta}^{(i)}=\boldsymbol{\theta}_{0}^{(i)}$. Without loss of generality, assume $i>1$. In view of (8.8), the first equality of (8.9) entails

$$
\sum_{j=1}^{i-1}\left\{\beta_{i j, t}\left(\varphi^{(+i)}\right)-\beta_{i j, t}\left(\varphi_{0}^{(+i)}\right)\right\} \sqrt{g_{j t}} \eta_{j t}=0 \quad \text { a.s. }
$$

Since the variables $\eta_{1 t}, \ldots, \eta_{i-1, t}$ are not linearly dependent, and the variables $g_{j t}$ are strictly positive (by $\mathbf{B 2}$ and $\mathbf{B 3}$ ), it follows that

$\beta_{i j, t}\left(\varphi^{(+i)}\right)=\beta_{i j, t}\left(\varphi_{0}^{(+i)}\right) \quad$ a.s., $\quad j=1, \ldots, i-1$.

Using $v_{i t}\left(\varphi^{(+i)}\right)=v_{i t}\left(\varphi_{0}^{(+i)}\right)$ in (2.11), (4.8), (8.9) and (8.10) entails

$$
\begin{aligned}
0= & \varpi_{i j}-\varpi_{0 i j}+\left(\varsigma_{i j+}-\varsigma_{0 i j+}\right) \epsilon_{1, t-1}^{+}+\left(\varsigma_{i j-}-\varsigma_{0 i j-}\right) \epsilon_{1, t-1}^{-} \\
& +\sum_{k=2}^{i}\left(\tau_{i j}^{(k)}-\tau_{0 i j}^{(k)}\right) v_{k, t-1}+\left(c_{i j}-c_{0 i j}\right) \beta_{i j, t-1} .
\end{aligned}
$$

We thus have

$$
\left(\tau_{i j}^{(i)}-\tau_{0 i j}^{(i)}\right) \eta_{i, t-1}=R_{t-1}^{(-i)} \text {, }
$$

where $R_{t-1}^{(-i)}$ is $\mathcal{F}_{t-1}^{(-i)}$-measurable. B2 then entails $\tau_{i j}^{(i)}=\tau_{0 i j}^{(i)}$. Continuing in this way, by $\mathbf{B 2}$ and $\mathbf{B} 4$ it follows that $\varphi^{(i j)}=\varphi_{0}^{(i j)}$, and finally we have shown that (8.9) implies $\vartheta_{0}^{(i)}=\boldsymbol{\vartheta}^{(i)}$. The remainder of the proof of the consistency of $\widehat{\vartheta}_{n}$ then follows from a standard compactness argument, as in the proof of Theorem 4.1.

Now we show the convergence in distribution (4.16). We first show that the effect of the initial values becomes asymptotically negligible. In Section 4.1.3, we have seen that, under B5, for $t \geq 2$

$\widetilde{\boldsymbol{\beta}}_{t}(\varphi)=\boldsymbol{w}_{t-1}^{*}+\boldsymbol{S}_{t-1} \widetilde{\boldsymbol{\beta}}_{t-1}(\varphi), \quad \boldsymbol{\beta}_{t}(\boldsymbol{\varphi})=\boldsymbol{w}_{t-1}^{*}+\boldsymbol{S}_{t-1} \boldsymbol{\beta}_{t-1}(\varphi)$,

with a fixed initial value $\widetilde{\boldsymbol{\beta}}_{1}(\boldsymbol{\varphi})$. Denoting by $\varphi_{k}$ the $k$ th element of $\varphi$, we thus have

$$
\begin{aligned}
& \frac{\partial \widetilde{\boldsymbol{\beta}}_{t}(\boldsymbol{\varphi})}{\partial \varphi_{k}}=\widetilde{\boldsymbol{w}}_{t-1}^{(k)}+\boldsymbol{S}_{t-1} \frac{\partial \widetilde{\boldsymbol{\beta}}_{t-1}(\boldsymbol{\varphi})}{\partial \varphi_{k}}, \\
& \frac{\partial \boldsymbol{\beta}_{t}(\boldsymbol{\varphi})}{\partial \varphi_{k}}=\boldsymbol{w}_{t-1}^{(k)}+\boldsymbol{S}_{t-1} \frac{\partial \boldsymbol{\beta}_{t-1}(\boldsymbol{\varphi})}{\partial \varphi_{k}},
\end{aligned}
$$

with

$\widetilde{\boldsymbol{w}}_{t}^{(k)}=\frac{\partial \boldsymbol{w}_{t}^{*}}{\partial \varphi_{k}}+\frac{\partial \boldsymbol{S}_{t}}{\partial \varphi_{k}} \widetilde{\boldsymbol{\beta}}_{t}(\boldsymbol{\varphi}), \quad \boldsymbol{w}_{t}^{(k)}=\frac{\partial \boldsymbol{w}_{t}^{*}}{\partial \varphi_{k}}+\frac{\partial \boldsymbol{S}_{t}}{\partial \varphi_{k}} \boldsymbol{\beta}_{t}(\boldsymbol{\varphi})$.

In view of (4.13), this entails that

$$
\sup _{\boldsymbol{\varphi} \in \Theta_{\varphi}}\left\|\widetilde{\boldsymbol{w}}_{t}^{(k)}-\boldsymbol{w}_{t}^{(k)}\right\| \leq K \rho_{t}, \quad \sup _{\boldsymbol{\varphi} \in \Theta_{\varphi}}\left\|\frac{\partial \widetilde{\boldsymbol{\beta}}_{t}(\boldsymbol{\varphi})}{\partial \boldsymbol{\varphi}^{\prime}}-\frac{\partial \boldsymbol{\beta}_{t}(\boldsymbol{\varphi})}{\partial \boldsymbol{\varphi}^{\prime}}\right\| \leq K \rho_{t},
$$

where $\rho_{t}$ is as in $\mathbf{A 2}$. It follows that

$\sup _{\boldsymbol{\vartheta} \in \Theta}\left\|\frac{\partial O_{n}(\boldsymbol{\vartheta})}{\partial \vartheta}-\frac{\partial \widetilde{O}_{n}(\boldsymbol{\vartheta})}{\partial \vartheta}\right\|=O\left(n^{-1}\right)$ a.s.

By the consistency and A6, we thus have

$0=\frac{1}{\sqrt{n}} \sum_{t=1}^{n} \frac{\partial}{\partial \vartheta} \widetilde{q}_{t}\left(\widehat{\vartheta}_{n}\right)=\frac{1}{\sqrt{n}} \sum_{t=1}^{n} \frac{\partial}{\partial \vartheta} q_{t}\left(\widehat{\vartheta}_{n}\right)+o_{P}(1)$

A Taylor expansion then gives

$$
\begin{aligned}
o_{P}(1)= & \frac{1}{\sqrt{n}} \sum_{t=1}^{n} \frac{\partial}{\partial \vartheta} q_{t}\left(\boldsymbol{\vartheta}_{0}\right) \\
& +\left[\frac{1}{n} \sum_{t=1}^{n} \frac{\partial^{2}}{\partial \vartheta_{k} \partial \vartheta_{j}} q_{t}\left(\boldsymbol{\vartheta}_{k l}\right)\right] \sqrt{n}\left(\widehat{\vartheta}_{n}-\vartheta_{0}\right),
\end{aligned}
$$

where, for the generic element $(k, j)$ of the matrix into brackets, $\boldsymbol{\vartheta}_{k l}$ is between $\boldsymbol{\vartheta}_{0}$ and $\widehat{\vartheta}_{n}$. Omitting the argument " $\left(\boldsymbol{\vartheta}^{(+i)}\right)$ " in $g_{i t}\left(\boldsymbol{\vartheta}^{(+i)}\right)$ and " $\left(\varphi^{(+i)}\right)$ " in $v_{i t}\left(\varphi^{(+i)}\right)$, for $i \geq 2$

$\frac{\partial q_{i t}\left(\boldsymbol{\vartheta}^{(+i)}\right)}{\partial \boldsymbol{\vartheta}^{(+i)}}=\left(1-\frac{v_{i t}^{2}}{g_{i t}}\right) \frac{1}{g_{i t}} \frac{\partial g_{i t}}{\partial \boldsymbol{\vartheta}^{(+i)}}+2 \frac{v_{i t}}{\sqrt{g_{i t}}} \frac{1}{\sqrt{g_{i t}}} \frac{\partial v_{i t}}{\partial \boldsymbol{\vartheta}^{(+i)}}$,

and thus

$\frac{\partial q_{i t}\left(\boldsymbol{\vartheta}_{0}^{(+i)}\right)}{\partial \boldsymbol{\vartheta}^{(+i)}}=\left(1-\eta_{i t}^{2}\right) \frac{1}{g_{i t}} \frac{\partial g_{i t}}{\partial \boldsymbol{\vartheta}^{(+i)}}\left(\boldsymbol{\vartheta}_{0}^{(+i)}\right)+2 \eta_{i t} \frac{1}{\sqrt{g_{i t}}} \frac{\partial v_{i t}}{\partial \boldsymbol{\vartheta}^{(+i)}}\left(\boldsymbol{\vartheta}_{0}^{(+i)}\right)$

\section{To establish that}

$\lim _{n \rightarrow \infty} \boldsymbol{I}_{n}=\boldsymbol{I}:=E \frac{\partial q_{t}\left(\boldsymbol{\vartheta}_{0}\right)}{\partial \boldsymbol{\vartheta}} \frac{\partial q_{t}\left(\boldsymbol{\vartheta}_{0}\right)}{\partial \boldsymbol{\vartheta}^{\prime}} \quad$ a.s.,

it suffices to use the ergodic theorem, the consistency of $\widehat{\vartheta}_{n}$, the arguments of Exercise 7.9 in Francq and Zakoïan (2010), and to show that for some neighborhood $V\left(\boldsymbol{\vartheta}_{0}\right)$ of $\boldsymbol{\vartheta}_{0}$

$E \sup _{\boldsymbol{\vartheta} \in V\left(\boldsymbol{\vartheta}_{0}\right)}\left\|\frac{\partial q_{t}(\boldsymbol{\vartheta})}{\partial \boldsymbol{\vartheta}} \frac{\partial q_{t}(\boldsymbol{\vartheta})}{\partial \boldsymbol{\vartheta}^{\prime}}\right\|<\infty$.

Note that $\mathbf{A 6}$ entails that the first $i+3$ components $\vartheta^{(+i)} \in$ $V\left(\vartheta_{0}^{(+i)}\right)$ are strictly positive, for any sufficiently small neighborhood $V\left(\boldsymbol{\vartheta}_{0}^{(+i)}\right)$ of $\boldsymbol{\vartheta}_{0}^{(+i)}$. By an extension of (5.20) in Hamadeh and Zakoïan (2011) and (42) in Francq and Thieu (2015), we then have

$$
\begin{aligned}
& E \sup _{\boldsymbol{\vartheta}^{(+i)} \in V\left(\vartheta_{0}^{(+i)}\right)}\left\|\frac{1}{g_{i t}} \frac{\partial g_{i t}\left(\boldsymbol{\vartheta}^{(+i)}\right)}{\partial \boldsymbol{\theta}^{(i)}}\right\|^{d}<\infty, \\
& E \sup _{\boldsymbol{\vartheta}^{(+i)} \in V\left(\vartheta_{0}^{(+i)}\right)}\left\|\frac{1}{g_{i t}} \frac{\partial^{2} g_{i t}\left(\boldsymbol{\vartheta}^{(+i)}\right)}{\partial \boldsymbol{\theta}^{(i)} \partial \boldsymbol{\theta}^{(i)^{\prime}}}\right\|^{d}<\infty,
\end{aligned}
$$

for any integer $d$ and some neighborhood $V\left(\boldsymbol{\vartheta}_{0}^{(+i)}\right)$ of $\boldsymbol{\vartheta}_{0}^{(+i)}$. Denote by $\varphi_{j}^{(+i)}$ the components of $\varphi^{(+i)}$, for $j=1, \ldots, i(i-1)(2 i+11) / 6$. Now, note that for $i \geq 2$

$$
\frac{\partial g_{i t}\left(\boldsymbol{\vartheta}^{(+i)}\right)}{\partial \varphi_{j}^{(+i)}}=2 \sum_{k=0}^{\infty} b_{i}^{k} \sum_{\ell=2}^{i} \alpha_{i}^{(\ell)} v_{\ell, t-k-1} \frac{\partial v_{\ell, t-k-1}\left(\boldsymbol{\varphi}^{(+\ell)}\right)}{\partial \varphi_{j}^{(+i)}} .
$$

For all $p_{1} \geq 1$, using the inequality $x /\left(1+x^{2}\right) \leq 1 / 2$ for all $x$, we thus have

$$
\begin{aligned}
\left\|\frac{1}{g_{i t}} \frac{\partial g_{i t}\left(\boldsymbol{\vartheta}^{(+i)}\right)}{\partial \varphi_{j}^{(i)}}\right\|_{p_{1}} & \leq 2 \sum_{k=0}^{\infty} \sum_{\ell=2}^{i}\left\|\frac{b_{i}^{k} \alpha_{i}^{(\ell)} v_{\ell, t-k-1} \frac{\partial v_{\ell, t-k-1}\left(\varphi^{(+\ell)}\right)}{\partial \varphi_{j}^{(+i)}}}{\omega_{i}+b_{i}^{k} \alpha_{i}^{(\ell)} v_{\ell, t-k-1}^{2}}\right\|_{p_{1}} \\
& \leq K \sum_{k=0}^{\infty} \rho^{k} \sum_{\ell=2}^{i}\left\|\frac{\partial v_{\ell, t-k-1}\left(\varphi^{(+\ell)}\right)}{\partial \varphi_{j}^{(+i)}}\right\|_{p_{1}} .
\end{aligned}
$$


In view of (8.15), the inequalities $\left\|\boldsymbol{v}_{t}(\boldsymbol{\varphi})\right\| \leq\left\|\boldsymbol{B}_{t}(\boldsymbol{\varphi})\right\|\left\|\boldsymbol{\epsilon}_{t}\right\|$ and $\left\|\frac{\partial \boldsymbol{v}_{t}(\boldsymbol{\varphi})}{\partial \varphi_{k}}\right\| \leq\left\|\frac{\partial \boldsymbol{B}_{t}(\boldsymbol{\varphi})}{\partial \varphi_{k}}\right\|\left\|\boldsymbol{\epsilon}_{t}\right\|$, and $\mathbf{B 6}$, for some neighborhood $V\left(\boldsymbol{\varphi}_{0}\right)$ of $\varphi_{0}$ we have

$E \sup _{\boldsymbol{\varphi} \in V\left(\boldsymbol{\varphi}_{0}\right)}\left\|\boldsymbol{v}_{t}(\boldsymbol{\varphi})\right\|^{4 p_{1}}<\infty$

$E \sup _{\varphi \in V\left(\varphi_{0}\right)}\left\|\frac{\partial \boldsymbol{v}_{t}(\boldsymbol{\varphi})}{\partial \boldsymbol{\varphi}}\right\|^{2 q_{1}}<\infty$,

$E \sup _{\boldsymbol{\vartheta}^{(+i)} \in V\left(\vartheta_{0}^{(+i)}\right)}\left\|\frac{1}{g_{i t}} \frac{\partial g_{i t}\left(\boldsymbol{\vartheta}^{(+i)}\right)}{\partial \boldsymbol{\vartheta}^{(+i)}}\right\|^{2 q_{1}}<\infty$.

In view of (8.12), we thus obtain (8.14) from the Hölder inequality, (8.6) and (8.16)-(8.17).

We now show that $\boldsymbol{I}$ is invertible. If this is not the case, then there exists a non zero vector $\lambda \in \mathbb{R}^{d}$ such that $\sum_{i=1}^{m} \lambda^{\prime} \frac{\partial q_{i t}\left(\vartheta_{0}^{(+i)}\right)}{\partial \vartheta}=0$ a.s., which implies

$\sum_{i=1}^{m}\left(1-\eta_{i t}^{2}\right) \frac{1}{g_{i t}} \lambda^{\prime} \frac{\partial g_{i t}}{\partial \boldsymbol{\vartheta}}\left(\boldsymbol{\vartheta}_{0}^{(+i)}\right)+2 \eta_{i t} \frac{1}{\sqrt{g_{i t}}} \lambda^{\prime} \frac{\partial v_{i t}}{\partial \vartheta}\left(\boldsymbol{\varphi}_{0}^{(+i)}\right)=0 \quad$ a.s.

By B2, this entails

$\lambda^{\prime} \frac{\partial g_{i t}}{\partial \vartheta}\left(\vartheta_{0}^{(+i)}\right)=0 \quad$ and

$\lambda^{\prime} \frac{\partial v_{i t}}{\partial \vartheta}\left(\varphi_{0}^{(+i)}\right)=0 \quad$ a.s. for $i=1, \ldots, m$.

Let the elimination matrices $\mathbf{E}_{\theta^{(i)}}^{0}$ and $\mathbf{E}_{\varphi^{(i j)}}^{0}$ (whose elements are zero or one) such that $\boldsymbol{\theta}^{(i)}=\mathbf{E}_{\boldsymbol{\theta}^{(i)}}^{0} \boldsymbol{\vartheta}$ and $\boldsymbol{\varphi}^{(i j)}=\mathbf{E}_{\boldsymbol{\varphi}^{(i j)}}^{0} \boldsymbol{\vartheta}$. Let $\boldsymbol{\lambda}_{\boldsymbol{\theta}^{(i)}}=$ $\mathbf{E}_{\theta^{(i)}}^{0} \lambda$ and $\lambda_{\varphi^{(i j)}}=\mathbf{E}_{\varphi^{(i j)}}^{0} \lambda$. With this notation, from (2.10) we have

$$
\begin{aligned}
& \lambda^{\prime} \frac{\partial g_{i t}}{\partial \boldsymbol{\vartheta}}\left(\boldsymbol{\vartheta}_{0}^{(+i)}\right)=\lambda_{\boldsymbol{\theta}^{(i)}}^{\prime} \frac{\partial}{\partial \boldsymbol{\theta}^{(i)}}\left\{\omega_{0 i}+\gamma_{0 i+}\left(\epsilon_{1, t-1}^{+}\right)^{2}+\gamma_{0 i-}\left(\epsilon_{1, t-1}^{-}\right)^{2}\right. \\
& \left.\quad+\sum_{k=2}^{i} \alpha_{0 i}^{(k)} v_{k, t-1}^{2}\left(\boldsymbol{\varphi}_{0}^{(+k)}\right)\right\} \\
& \quad+2 \sum_{k=2}^{i} \alpha_{0 i}^{(k)} v_{k, t-1} \lambda^{\prime} \frac{\partial v_{k, t-1}}{\partial \boldsymbol{\vartheta}}\left(\boldsymbol{\varphi}_{0}^{(+k)}\right)+\lambda^{\prime} \frac{\partial g_{i, t-1}}{\partial \boldsymbol{\vartheta}}\left(\boldsymbol{\vartheta}_{0}^{(+i)}\right) .
\end{aligned}
$$

Therefore (8.18) entails

$$
\left(1\left(\epsilon_{t-1}^{+}\right)^{2}\left(\epsilon_{t-1}^{-}\right)^{2} v_{2, t-1}^{2} \cdots v_{i, t-1}^{2} g_{i, t-1}\right) \lambda_{\boldsymbol{\theta}^{(i)}}=0 .
$$

Then, as in Page 163 of Francq and Zakoïan (2010), it can be shown that $\mathbf{B 2}$ and $\mathbf{B} 4$ imply that $\lambda_{\theta^{(i)}}=\mathbf{0}$. Now note that the second equality of (8.18) implies

$0=\lambda_{\varphi^{(i j)}}^{\prime} \frac{\partial v_{i t}}{\partial \varphi^{(i j)}}\left(\varphi_{0}^{(+i)}\right)=-\sum_{\ell=1}^{i-1} \epsilon_{\ell, t} \lambda_{\varphi^{(i j)}}^{\prime} \frac{\partial \beta_{i \ell, t}}{\partial \varphi^{(i j)}}\left(\varphi_{0}^{(+i)}\right)$.

When $\ell \neq j$, we have

$$
\begin{aligned}
\lambda_{\varphi^{(i j)}}^{\prime} \frac{\partial \beta_{i \ell, t}}{\partial \varphi^{(i j)}}\left(\varphi_{0}^{(+i)}\right)= & \tau_{0 i \ell}^{(i)} \lambda_{\varphi^{(i j)}}^{\prime} \frac{\partial v_{i, t-1}}{\partial \varphi^{(i j)}}\left(\varphi_{0}^{(+i)}\right) \\
& +c_{0 i \ell} \lambda_{\varphi^{(i j)}}^{\prime} \frac{\partial \beta_{i \ell, t-1}}{\partial \varphi^{(i j)}}\left(\varphi_{0}^{(+i)}\right) \\
= & c_{0 i \ell} \lambda_{\varphi^{(i j)}} \frac{\partial \beta_{i \ell, t-1}}{\partial \varphi^{(i j)}}\left(\varphi_{0}^{(+i)}\right)=0
\end{aligned}
$$

by stationarity. By B2, we thus have

$$
\begin{aligned}
0 & =\lambda_{\varphi^{(i j)}}^{\prime} \frac{\partial \beta_{i j, t}}{\partial \varphi^{(i j)}}\left(\varphi_{0}^{(+i)}\right) \\
& =\left(1 \epsilon_{t-1}^{+} \epsilon_{t-1}^{-} v_{2, t-1} \cdots v_{i, t-1} \beta_{i j, t-1}\right) \lambda_{\varphi^{(i j)}} .
\end{aligned}
$$

We then show that $\lambda_{\varphi^{(i j)}}=\mathbf{0}$ from B2 and B4. Since the previous results are true for all $i \in\{1, \ldots, m\}$ and all $(i, j) \in \boldsymbol{T}_{m}$, we have shown that $\lambda=\mathbf{0}$, which proves the invertibility by contradiction.

Differentiating (8.12), we obtain

$$
\begin{aligned}
\frac{\partial^{2} q_{i t}\left(\boldsymbol{\vartheta}^{(+i)}\right)}{\partial \boldsymbol{\vartheta}^{(+i)} \partial \boldsymbol{\vartheta}^{(+i)^{\prime}}}= & \left(1-\frac{v_{i t}^{2}}{g_{i t}}\right) \frac{1}{g_{i t}} \frac{\partial^{2} g_{i t}}{\partial \boldsymbol{\vartheta}^{(+i)} \partial \boldsymbol{\vartheta}^{(+i)^{\prime}}} \\
& +\left(\frac{2 v_{i t}^{2}}{g_{i t}}-1\right) \frac{1}{g_{i t}} \frac{\partial g_{i t}}{\partial \boldsymbol{\vartheta}^{(+i)}} \frac{1}{g_{i t}} \frac{\partial g_{i t}}{\partial \boldsymbol{\vartheta}^{(+i)^{\prime}}} \\
& +\frac{2}{g_{i t}} \frac{\partial v_{i t}}{\partial \boldsymbol{\vartheta}^{(+i)}} \frac{\partial v_{i t}}{\partial \boldsymbol{\vartheta}^{(+i)^{\prime}}}+\frac{2 v_{i t}}{g_{i t}} \frac{\partial^{2} v_{i t}}{\partial \boldsymbol{\vartheta}^{(+i)} \partial \boldsymbol{\vartheta}^{(+i)^{\prime}}} \\
& -\frac{2 v_{i t}}{g_{i t}}\left\{\frac{\partial v_{i t}}{\partial \boldsymbol{\vartheta}^{(+i)}} \frac{1}{g_{i t}} \frac{\partial g_{i t}}{\partial \boldsymbol{\vartheta}^{(+i)^{\prime}}}+\frac{1}{g_{i t}} \frac{\partial g_{i t}}{\partial \boldsymbol{\vartheta}^{(+i)}} \frac{\partial v_{i t}}{\partial \boldsymbol{\vartheta}^{(+i)^{\prime}}}\right\} .
\end{aligned}
$$

We thus have

$\boldsymbol{J}:=\sum_{i=1}^{m} E \frac{\partial^{2} q_{i t}\left(\boldsymbol{\vartheta}_{0}^{(+i)}\right)}{\partial \boldsymbol{\vartheta} \partial \boldsymbol{\vartheta}^{\prime}}=\sum_{i=1}^{m} E \frac{1}{g_{i t}} \frac{\partial g_{i t}}{\partial \boldsymbol{\vartheta}} \frac{1}{g_{i t}} \frac{\partial g_{i t}}{\partial \boldsymbol{\vartheta}^{\prime}}+2 E \frac{1}{g_{i t}} \frac{\partial v_{i t}}{\partial \boldsymbol{\vartheta}} \frac{\partial v_{i t}}{\partial \boldsymbol{\vartheta}^{\prime}}$.

The invertibility of $\boldsymbol{J}$ and the convergence of $\boldsymbol{J}_{n}$ to $\boldsymbol{J}$ is shown by already given arguments. The conclusion follows from the central limit theorem for squared integrable martingale differences.

Proof of Theorem 4.3. The consistency of $\widehat{\vartheta}_{n}^{(1)}$ and $\widehat{\vartheta}_{n}^{(2)}$ is obtained exactly as the consistency in Theorem 4.2. We then continue the proof by induction on $i$, and assume that $\widehat{\vartheta}_{n}^{(k)} \rightarrow \boldsymbol{\vartheta}_{0}^{(k)}$ a.s. for $k=$ $1, \ldots, i-1$. Let $V^{(-i)}\left(\varphi_{0}^{(-i)}\right)$ and $V^{(i)}\left(\vartheta_{0}^{(i)}\right)$ be arbitrary neighborhoods of $\varphi_{0}^{(-i)}$ and $\vartheta_{0}^{(i)}$, and let

$V^{(+i)}\left(\vartheta_{0}^{(+i)}\right)=V^{(i)}\left(\vartheta_{0}^{(i)}\right) \times V^{(-i)}\left(\varphi_{0}^{(-i)}\right)$

be the corresponding neighborhood of $\boldsymbol{\vartheta}_{0}^{(+i)}$. For any $\boldsymbol{\vartheta}_{1}^{(i)} \neq \boldsymbol{\vartheta}_{0}^{(i)}$, by (8.9) and arguments given in the proof of Theorem 4.1, there exists a neighborhood $V^{(i)}\left(\vartheta_{1}^{(i)}\right)$ of $\vartheta_{1}^{(i)}$ such that

$$
\begin{aligned}
& \liminf _{n \rightarrow \infty} \frac{1}{n} \sum_{t=1}^{n} \inf _{\vartheta^{(i)} \in V^{(i)}\left(\vartheta_{1}^{(i)}\right)} q_{i t}\left(\boldsymbol{\vartheta}^{(i)}, \widehat{\boldsymbol{\varphi}}_{n}^{(-i)}\right) \\
& \geq E \inf _{\boldsymbol{\vartheta}^{(+i)} \in V^{(i)}\left(\vartheta_{1}^{(i)}\right) \times V^{(-i)}\left(\boldsymbol{\varphi}_{0}^{(-i)}\right)} q_{i t}\left(\boldsymbol{\vartheta}^{(+i)}\right)>E q_{i t}\left(\boldsymbol{\vartheta}_{0}^{(+i)}\right) .
\end{aligned}
$$

By compactness of the parameter space, this entails that, asymptotically, $\widehat{\vartheta}_{n}^{(i)}$ belongs to $V^{(i)}\left(\boldsymbol{\vartheta}_{0}^{(i)}\right)$, which completes the proof of the consistency.

Now we show the convergence in distribution (4.20)-(4.21). For $i=1$, the result comes from already known results on the QMLE of univariate APARCH models (see Hamadeh and Zakoïan, 2011). Note that in the case $i=2$ the EbEE still coincides with the one-step QMLE (see Section 4.2). Therefore (4.20) follows from Theorem 4.2 applied with $m=2$. More precisely, in the proof of this theorem, we have shown that for $j=2$ one has the Bahadur representation

$$
\sqrt{n}\left(\begin{array}{c}
\widehat{\boldsymbol{\vartheta}}_{n}^{(j)}-\boldsymbol{\vartheta}_{0}^{(j)} \\
\widehat{\boldsymbol{\varphi}}_{n}^{(-j)}-\boldsymbol{\varphi}_{0}^{(-j)}
\end{array}\right) \stackrel{o_{P}^{(1)}}{=} \boldsymbol{M}^{(j)} \frac{1}{\sqrt{n}} \sum_{t=1}^{n}\left(\begin{array}{c}
\frac{\partial q_{j t}\left(\boldsymbol{\vartheta}_{0}^{(+j)}\right)}{\partial \boldsymbol{\vartheta}^{(j)}} \\
\frac{\partial q_{j-1, t}\left(\boldsymbol{\vartheta}_{0}^{(+(j-1))}\right)}{\partial \boldsymbol{\varphi}^{(j-1)}} \\
\vdots \\
\frac{\partial q_{2 t}\left(\boldsymbol{\vartheta}_{0}^{(2)}\right)}{\partial \boldsymbol{\varphi}^{(2)}}
\end{array}\right)
$$

for some matrix of the form

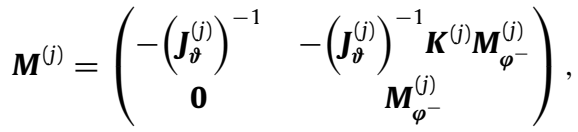

and the convention $\boldsymbol{M}^{(2)}=-\left(\boldsymbol{J}_{\vartheta}^{(2)}\right)^{-1}$. 
We now show (8.19) by induction on $j$, for $3 \leq j \leq m$. Similarly to (8.11), one can show that

$$
\sup _{\boldsymbol{\vartheta}^{(+i)} \in \Theta^{(+i)}}\left\|\frac{\partial O_{n}^{(i)}\left(\boldsymbol{\vartheta}^{(+i)}\right)}{\partial \boldsymbol{\vartheta}^{(+i)}}-\frac{\partial \widetilde{O}_{n}^{(i)}\left(\boldsymbol{\vartheta}^{(+i)}\right)}{\partial \boldsymbol{\vartheta}^{(+i)}}\right\|=O\left(n^{-1}\right) \text { a.s. }
$$

We have

$$
\begin{aligned}
0 & =\frac{1}{\sqrt{n}} \sum_{t=1}^{n} \frac{\partial}{\partial \boldsymbol{\vartheta}^{(i)}} \widetilde{q}_{i t}\left(\widehat{\boldsymbol{\vartheta}}_{n}^{(i)}, \widehat{\boldsymbol{\varphi}}_{n}^{(-i)}\right) \\
& =\frac{1}{\sqrt{n}} \sum_{t=1}^{n} \frac{\partial}{\partial \boldsymbol{\vartheta}^{(i)}} q_{i t}\left(\widehat{\vartheta}_{n}^{(i)}, \widehat{\boldsymbol{\varphi}}_{n}^{(-i)}\right)+o_{P}(1),
\end{aligned}
$$

where the first equality comes from $\mathbf{A 6}$ and the consistency, and the second one from (8.20). A first Taylor expansion thus gives

$$
\begin{aligned}
o_{P}(1)= & \frac{1}{\sqrt{n}} \sum_{t=1}^{n} \frac{\partial q_{i t}\left(\boldsymbol{\vartheta}_{0}^{(i)}, \widehat{\boldsymbol{\varphi}}_{n}^{(-i)}\right)}{\partial \boldsymbol{\vartheta}^{(i)}} \\
& +\left[\frac{1}{n} \sum_{t=1}^{n} \frac{\partial^{2} q_{i t}\left(\boldsymbol{\vartheta}_{k l}^{(+i)}\right)}{\partial \vartheta_{k}^{(i)} \partial \vartheta_{j}^{(i)}}\right] \sqrt{n}\left(\widehat{\vartheta}_{n}^{(i)}-\boldsymbol{\vartheta}_{0}^{(i)}\right),
\end{aligned}
$$

where, for the generic element $(k, j)$ of the matrix into brackets, $\vartheta_{k l}^{(+i)}$ is between $\vartheta_{0}^{(+i)}$ and $\widehat{\vartheta}_{n}^{(+i)}$. Another Taylor expansion yields

$$
\begin{aligned}
& \frac{1}{\sqrt{n}} \sum_{t=1}^{n} \frac{\partial q_{i t}\left(\boldsymbol{\vartheta}_{0}^{(i)}, \widehat{\boldsymbol{\varphi}}_{n}^{(-i)}\right)}{\partial \boldsymbol{\vartheta}^{(i)}}=\frac{1}{\sqrt{n}} \sum_{t=1}^{n} \frac{\partial q_{i t}\left(\boldsymbol{\vartheta}_{0}^{(+i)}\right)}{\partial \boldsymbol{\vartheta}^{(i)}} \\
& +\left[\frac{1}{n} \sum_{t=1}^{n} \frac{\partial^{2} q_{i t}\left(\boldsymbol{\vartheta}_{0}^{(i)}, \boldsymbol{\varphi}_{k l}^{(-i)}\right)}{\partial \vartheta_{k}^{(i)} \partial \varphi_{j}^{(-i)}}\right] \sqrt{n}\left(\widehat{\boldsymbol{\varphi}}_{n}^{(-i)}-\boldsymbol{\varphi}_{0}^{(-i)}\right),
\end{aligned}
$$

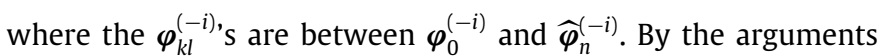
used to prove that $\boldsymbol{I}_{n}$ and $\boldsymbol{J}_{n}$ converge to $\boldsymbol{I}$ and $\boldsymbol{J}$ in Theorem 4.2, one can show that

$$
\lim _{n \rightarrow \infty} \boldsymbol{J}_{n}^{(i)}=\lim _{n \rightarrow \infty}\left[\frac{1}{n} \sum_{t=1}^{n} \frac{\partial^{2} q_{i t}\left(\boldsymbol{\vartheta}_{k l}^{(+i)}\right)}{\partial \vartheta_{k}^{(i)} \partial \vartheta_{j}^{(i)}}\right]=\boldsymbol{J}^{(i)}
$$

and

$$
\lim _{n \rightarrow \infty} \boldsymbol{K}_{n}^{(i)}=\lim _{n \rightarrow \infty}\left[\frac{1}{n} \sum_{t=1}^{n} \frac{\partial^{2} q_{i t}\left(\boldsymbol{\vartheta}_{0}^{(i)}, \boldsymbol{\varphi}_{k l}^{(-i)}\right)}{\partial \vartheta_{k}^{(i)} \partial \varphi_{j}^{(-i)}}\right]=\boldsymbol{K}^{(i)},
$$

where

$\boldsymbol{J}^{(i)}=E \frac{1}{g_{i t}} \frac{\partial g_{i t}}{\partial \boldsymbol{\vartheta}^{(i)}} \frac{1}{g_{i t}} \frac{\partial g_{i t}}{\partial \boldsymbol{\vartheta}^{(i)^{\prime}}}\left(\boldsymbol{\vartheta}_{0}^{(+i)}\right)+2 E \frac{1}{g_{i t}} \frac{\partial v_{i t}}{\partial \boldsymbol{\vartheta}^{(i)}} \frac{\partial v_{i t}}{\partial \boldsymbol{\vartheta}^{(i)^{\prime}}}\left(\boldsymbol{\vartheta}_{0}^{(+i)}\right)$

is a positive definite matrix, and

$$
\boldsymbol{K}^{(i)}=E \frac{\partial^{2} q_{i t}\left(\vartheta_{0}^{(+i)}\right)}{\partial \boldsymbol{\vartheta}^{(i)} \partial \boldsymbol{\varphi}^{(-i)}} \text {. }
$$

We thus have

$$
\begin{aligned}
& \sqrt{n}\left(\widehat{\boldsymbol{\vartheta}}_{n}^{(i)}-\boldsymbol{\vartheta}_{0}^{(i)}\right) \stackrel{o_{P}(1)}{=}-\left\{\boldsymbol{J}^{(i)}\right\}^{-1} \\
& \quad \times\left\{\frac{1}{\sqrt{n}} \sum_{t=1}^{n} \frac{\partial q_{i t}\left(\boldsymbol{\vartheta}_{0}^{(+i)}\right)}{\partial \boldsymbol{\vartheta}^{(i)}}+\boldsymbol{K}^{(i)} \sqrt{n}\left(\widehat{\boldsymbol{\varphi}}_{n}^{(-i)}-\boldsymbol{\varphi}_{0}^{(-i)}\right)\right\} .
\end{aligned}
$$

By induction, one can assume that

$$
\begin{aligned}
\sqrt{n}\left(\widehat{\boldsymbol{\varphi}}_{n}^{(-i)}-\boldsymbol{\varphi}_{0}^{(-i)}\right) & =\sqrt{n}\left(\widehat{\boldsymbol{\varphi}}_{n}^{(+(i-1))}-\boldsymbol{\varphi}_{0}^{(+(i-1))}\right) \\
& =\boldsymbol{M}_{\boldsymbol{\varphi}^{-}}^{(i)} \frac{1}{\sqrt{n}} \sum_{t=1}^{n}\left(\begin{array}{c}
\frac{\partial q_{i-1, t}\left(\boldsymbol{\vartheta}_{0}^{(+(i-1))}\right)}{\partial \boldsymbol{\varphi}^{(i-1)}} \\
\vdots \\
\frac{\partial q_{2 t}\left(\boldsymbol{\vartheta}_{0}^{(2)}\right)}{\partial \boldsymbol{\varphi}^{(2)}}
\end{array}\right),
\end{aligned}
$$

which holds true for $i-1=2$. It follows that (4.2) holds for $j=i$. By (8.13), we have

$$
\frac{1}{\sqrt{n}} \sum_{t=1}^{n}\left(\begin{array}{c}
\frac{\partial q_{j t}\left(\boldsymbol{\vartheta}_{0}^{(+j)}\right)}{\partial \boldsymbol{\vartheta}^{(j)}} \\
\frac{\partial q_{i-1, t}\left(\boldsymbol{\vartheta}_{0}^{(+(i-1))}\right)}{\partial \boldsymbol{\varphi}^{(i-1)}} \\
\vdots \\
\frac{\partial q_{2 t}\left(\boldsymbol{\vartheta}_{0}^{(2)}\right)}{\partial \boldsymbol{\varphi}^{(2)}}
\end{array}\right) \stackrel{\mathcal{L}}{\rightarrow} \mathcal{N}\left(0, \boldsymbol{I}^{(+i)}\right) .
$$

Under B7, the matrix $\mathbf{I}^{(+i)}$ is block-diagonal, and the conclusion follows.

Proof of Theorem 4.4. Note that $\mathbf{A 1}$ follows from (4.22) and the compactness of $\Theta$. By (4.23) we have

$$
\begin{aligned}
& \left|g_{i t}(\boldsymbol{\vartheta})-\widetilde{g}_{i t}(\boldsymbol{\vartheta})\right|=b_{i}\left|g_{i, t-1}(\boldsymbol{\vartheta})-\widetilde{g}_{i, t-1}(\boldsymbol{\vartheta})\right| \leq K \rho^{t}, \\
& \left|\beta_{i j, t}(\boldsymbol{\vartheta})-\widetilde{\beta}_{i j, t}(\boldsymbol{\vartheta})\right| \leq K \rho^{t},
\end{aligned}
$$

thus A2 holds true. The $c_{r}$-inequality, $E\left\|\boldsymbol{\epsilon}_{t}\right\|^{s_{0}}<\infty$ and (4.23) entail A3. Assumption A5 is obviously satisfied. The ergodicity then entails that

$\lim _{n \rightarrow \infty} \frac{1}{n} \sum_{t=1}^{n} \widetilde{q}_{t}(\boldsymbol{\vartheta})=E q_{1}(\boldsymbol{\vartheta}) \quad$ a.s., $\quad q_{t}(\boldsymbol{\vartheta})=\sum_{i=1}^{m} q_{i t}\left(\boldsymbol{\vartheta}^{(i)}\right)$,

with, for $t=1, \ldots, n$,

$$
\begin{aligned}
q_{i t}\left(\boldsymbol{\vartheta}^{(i)}\right)= & \frac{v_{i t}^{2}\left(\boldsymbol{\varphi}^{(i)}\right)}{g_{i t}\left(\boldsymbol{\theta}^{(i)}\right)}+\log g_{i t}\left(\boldsymbol{\theta}^{(i)}\right), \\
g_{i t}\left(\boldsymbol{\theta}^{(i)}\right)= & \omega_{i}+\sum_{k=1}^{m}\left\{\alpha_{i+}^{(k)}\left(\epsilon_{k, t-1}^{+}\right)^{2}+\alpha_{i-}^{(k)}\left(\epsilon_{k, t-1}^{-}\right)^{2}\right\} \\
& +b_{i} g_{i, t-1}\left(\boldsymbol{\theta}^{(i)}\right), \\
v_{i t}\left(\boldsymbol{\varphi}^{(i)}\right)= & \epsilon_{i t}-\sum_{j=1}^{i-1} \beta_{i j, t}\left(\boldsymbol{\varphi}^{(i j)}\right) \epsilon_{j t}, \\
\beta_{i j, t}\left(\boldsymbol{\varphi}^{(i j)}\right)= & \varpi_{i j}+\tau_{i j} \epsilon_{i, t-1} \epsilon_{j, t-1}+c_{i j} \beta_{i j, t-1}\left(\boldsymbol{\varphi}^{(i j)}\right),
\end{aligned}
$$

using the notation $\vartheta=\left(\vartheta^{(1)^{\prime}}, \ldots, \vartheta^{(m)^{\prime}}\right)^{\prime}$,

$$
\boldsymbol{\vartheta}^{(i)}=\left(\boldsymbol{\theta}^{(i)^{\prime}}, \boldsymbol{\varphi}^{(i)^{\prime}}\right)^{\prime}, \quad \boldsymbol{\theta}^{(i)}=\left(\omega_{i}, \alpha_{i+}^{(1)}, \ldots, \alpha_{i-}^{(m)}, b_{i}\right)^{\prime},
$$$$
\varphi^{(i)}=\left(\varphi^{(i 1)}, \ldots, \varphi^{(i, i-1)}\right)^{\prime},
$$

$\varphi^{(i j)}=\left(\varpi_{i j}, \tau_{i j}, c_{i j}\right)^{\prime}$, and obvious conventions, such as $v_{1 t}\left(\varphi^{(1)}\right)=$ $\epsilon_{1 t}$ or $\boldsymbol{\vartheta}^{(1)}=\boldsymbol{\theta}^{(1)}$. By an abuse of notation, we are now writing for instance $\beta_{i j, t}(\vartheta)=\beta_{i j, t}\left(\varphi^{(i)}\right)=\beta_{i j, t}\left(\varphi^{(i j)}\right)$. Similarly to (8.9), one can show that $E q_{i t}\left(\vartheta^{(i)}\right) \geq E q_{i t}\left(\vartheta_{0}^{(i)}\right)$ with equality if and only if

$v_{i t}\left(\varphi^{(i)}\right)=v_{i t}\left(\varphi_{0}^{(i)}\right) \quad$ and $\quad g_{i t}\left(\boldsymbol{\theta}^{(i)}\right)=g_{i t}\left(\boldsymbol{\theta}_{0}^{(i)}\right) \quad$ a.s.

With the notation of the proof of Theorem 4.2 , the second equality entails

$$
\left(\alpha_{0 i+}^{(i)}-\alpha_{i+}^{(i)}\right)\left(\eta_{i, t-1}^{+}\right)^{2}+\left(\alpha_{0 i-}^{(i)}-\alpha_{i-}^{(i)}\right)\left(\eta_{i, t-1}^{-}\right)^{2}=R_{t-1}^{(-i)}
$$

By B2* ${ }^{*}$, this implies $\alpha_{0 i+}^{(i)}=\alpha_{i+}^{(i)}$ and $\alpha_{0 i-}^{(i)}=\alpha_{i-}^{(i)}$. Continuing in this way, we show that, under $\mathbf{B 2}{ }^{*}$ and $\mathbf{B} 4^{*}$, the relation $g_{i t}\left(\boldsymbol{\theta}^{(i)}\right)=$ $g_{i t}\left(\boldsymbol{\theta}_{0}^{(i)}\right)$ a.s. entails $\boldsymbol{\theta}^{(i)}=\boldsymbol{\theta}_{0}^{(i)}$. Now, similarly to (8.10), the first equality of (8.21) entails, for $j=1, \ldots, i-1, \beta_{i j, t}\left(\varphi^{(i j)}\right)=\beta_{i j, t}\left(\varphi_{0}^{(i j)}\right)$. This implies

$\left(\pi_{i j}-\pi_{0 i j}\right) \eta_{i, t-1} \eta_{j, t-1}=R_{t-2}$,

which entails $\pi_{i j}=\pi_{0 i j}$ by the last part of $\mathbf{B 2}{ }^{*}$. Continuing this way, we finally conclude that (8.21) implies $\vartheta^{(i)}=\vartheta_{0}^{(i)}$. The consistency then follows as in the proof of Theorem 4.1. 
Now we turn to the asymptotic distribution. First note that A7 and $\mathbf{A 8}$ are satisfied, with $\rho_{t}=\rho^{t}$. It follows that we have a result of the form (8.11), showing that the initial values are asymptotically unimportant. Now, similarly to (8.13), we have

$$
\begin{aligned}
\frac{\partial q_{i t}\left(\boldsymbol{\vartheta}_{0}^{(i)}\right)}{\partial \boldsymbol{\vartheta}^{(i)}}= & \left(1-\eta_{i t}^{2}\right)\left(\begin{array}{c}
\frac{1}{g_{i t}} \frac{\partial g_{i t}}{\partial \boldsymbol{\theta}^{(i)}}\left(\boldsymbol{\theta}_{0}^{(i)}\right) \\
\mathbf{0}
\end{array}\right) \\
& +2 \eta_{i t}\left(\begin{array}{c}
\frac{1}{\sqrt{g_{i t}}} \frac{\partial v_{i t}}{\partial \boldsymbol{\varphi}^{(i)}}\left(\boldsymbol{\varphi}_{0}^{(i)}\right)
\end{array}\right) .
\end{aligned}
$$

We also have

$$
\begin{aligned}
\frac{\partial^{2} q_{i t}\left(\boldsymbol{\vartheta}^{(i)}\right)}{\partial \boldsymbol{\theta}^{(i)} \partial \boldsymbol{\theta}^{(i)^{\prime}}}= & \left(1-\frac{v_{i t}^{2}}{g_{i t}}\right) \frac{1}{g_{i t}} \frac{\partial^{2} g_{i t}}{\partial \boldsymbol{\theta}^{(i)} \partial \boldsymbol{\theta}^{(i)^{\prime}}} \\
& +\left(\frac{2 v_{i t}^{2}}{g_{i t}}-1\right) \frac{1}{g_{i t}} \frac{\partial g_{i t}}{\partial \boldsymbol{\theta}^{(i)}} \frac{1}{g_{i t}} \frac{\partial g_{i t}}{\partial \boldsymbol{\theta}^{(i)^{\prime}}}, \\
\frac{\partial^{2} q_{i t}\left(\boldsymbol{\vartheta}^{(i)}\right)}{\partial \boldsymbol{\theta}^{(i)} \partial \boldsymbol{\varphi}^{(i)^{\prime}}}= & -\frac{2 v_{i t}}{g_{i t}} \frac{1}{g_{i t}} \frac{\partial g_{i t}}{\partial \boldsymbol{\theta}^{(i)}} \frac{\partial v_{i t}}{\partial \boldsymbol{\varphi}^{(i)^{\prime}}}, \\
\frac{\partial^{2} q_{i t}\left(\boldsymbol{\vartheta}^{(i)}\right)}{\partial \boldsymbol{\varphi}^{(i)} \partial \boldsymbol{\varphi}^{(i)^{\prime}}}= & \frac{2}{g_{i t}} \frac{\partial v_{i t}}{\partial \boldsymbol{\varphi}^{(i)}} \frac{\partial v_{i t}}{\partial \boldsymbol{\varphi}^{(i)^{\prime}}}+\frac{2 v_{i t}}{g_{i t}} \frac{\partial^{2} v_{i t}}{\partial \boldsymbol{\varphi}^{(i)} \partial \boldsymbol{\varphi}^{(i)^{\prime}}} .
\end{aligned}
$$

Note that for any $d$

$$
\begin{aligned}
& E \sup _{\boldsymbol{\theta}^{(i)} \in V\left(\boldsymbol{\theta}_{0}^{(i)}\right)}\left\|\frac{1}{g_{i t}\left(\boldsymbol{\theta}^{(i)}\right)} \frac{\partial g_{i t}\left(\boldsymbol{\theta}^{(i)}\right)}{\partial \boldsymbol{\theta}^{(i)}}\right\|^{d}<\infty, \\
& E \sup _{\boldsymbol{\theta}^{(i)} \in V\left(\boldsymbol{\theta}_{0}^{(i)}\right)}\left\|\frac{1}{g_{i t}\left(\boldsymbol{\theta}^{(i)}\right)} \frac{\partial^{2} g_{i t}\left(\boldsymbol{\theta}^{(i)}\right)}{\partial \boldsymbol{\theta}^{(i)} \partial \boldsymbol{\theta}^{(i)^{\prime}}}\right\|^{d}<\infty
\end{aligned}
$$

for some neighborhood $V\left(\boldsymbol{\theta}_{0}^{(i)}\right)$ of $\boldsymbol{\theta}_{0}^{(i)}$ (by arguments similar to those showing (8.15)). Writing for instance " $\varphi^{(i j)} \in \Theta$ " instead of " $\varphi^{(i j)}$ such that $\vartheta \in \Theta$ ”, the compactness of $\Theta$, (4.23), B3* and Hölder's inequality entail

$E \sup _{\vartheta \in \Theta}\left\|\beta_{i j, t}\left(\varphi^{(i j)}\right)\right\|^{s_{0} / 2}<\infty, \quad E \sup _{\vartheta \in \Theta}\left\|\frac{\partial \beta_{i j, t}\left(\varphi^{(i j)}\right)}{\partial \varphi^{(i j)}}\right\|^{s_{0} / 2}<\infty$,

and

$$
\begin{aligned}
& E \sup _{\vartheta \in \Theta}\left|v_{i t}\left(\boldsymbol{\varphi}^{(i)}\right)\right|^{S_{0} / 3}<\infty, \\
& \left\|\sup _{\vartheta \in \Theta}\right\| \frac{\partial v_{i t}\left(\varphi^{(i)}\right)}{\partial \varphi^{(i j)}}\|\|_{s_{0} / 3}=\left\|\left|\epsilon_{j t}\right| \sup _{\vartheta \in \Theta}\right\| \frac{\partial \beta_{i j, t}\left(\varphi^{(i j)}\right)}{\partial \varphi^{(i j)}}\|\|_{s_{0} / 3}<\infty .
\end{aligned}
$$

We also have $\operatorname{Esup}_{\boldsymbol{\vartheta} \in \Theta}\left\|\frac{\partial^{2} v_{i t}\left(\varphi^{(i)}\right)}{\partial \varphi^{(i j)} \partial \varphi^{(i j)^{\prime}}}\right\|^{s_{0} / 3}<\infty$. By the central limit theorem for squared integrable martingale differences and the ergodic theorem, we then deduce that there exist matrices $I$ and $J$ such that

$$
\frac{1}{\sqrt{n}} \sum_{t=1}^{n} \frac{\partial q_{t}\left(\boldsymbol{\vartheta}_{0}\right)}{\partial \boldsymbol{\vartheta}} \stackrel{\mathcal{L}}{\rightarrow} \mathcal{N}(0, \boldsymbol{I}), \quad \frac{1}{n} \sum_{t=1}^{n} \frac{\partial^{2} q_{t}\left(\widehat{\vartheta}_{n}\right)}{\partial \boldsymbol{\vartheta} \partial \boldsymbol{\vartheta}^{\prime}} \rightarrow \boldsymbol{J},
$$

and we conclude as in the proof of Theorem 4.2. The matrix $\boldsymbol{J}$ is block-diagonal, of the form

$\boldsymbol{J}=\operatorname{diag}\left(\boldsymbol{J}_{\boldsymbol{\theta}}^{(1)}, \boldsymbol{J}_{\boldsymbol{\theta}}^{(2)}, \boldsymbol{J}_{\varphi}^{(2)}, \ldots, \boldsymbol{J}_{\boldsymbol{\theta}}^{(m)}, \boldsymbol{J}_{\varphi}^{(m)}\right)$

with

$\boldsymbol{J}_{\boldsymbol{\theta}}^{(i)}=E \frac{1}{g_{i t}^{2}} \frac{\partial g_{i t}}{\partial \boldsymbol{\theta}^{(i)}} \frac{\partial g_{i t}}{\partial \boldsymbol{\theta}^{(i)^{\prime}}}\left(\boldsymbol{\theta}_{0}^{(i)}\right), \quad \boldsymbol{J}_{\varphi}^{(i)}=2 E \frac{1}{g_{i t}} \frac{\partial v_{i t}}{\partial \boldsymbol{\varphi}^{(i)}} \frac{\partial v_{i t}}{\partial \boldsymbol{\varphi}^{(i)^{\prime}}}\left(\boldsymbol{\varphi}_{0}^{(i)}\right)$.

Note that, when the components of $\eta_{t}$ are independent and symmetric (or more generally when $E \eta_{i t}^{2} \eta_{j t}^{2}=1$ and $E \eta_{i t}^{3}=0$ ), the matrix $\boldsymbol{I}$ is also of the block-diagonal form

$\boldsymbol{I}=\operatorname{diag}\left(\boldsymbol{I}_{\boldsymbol{\theta}}^{(1)}, \boldsymbol{I}_{\boldsymbol{\theta}}^{(2)}, \boldsymbol{I}_{\boldsymbol{\varphi}}^{(2)}, \ldots, \boldsymbol{I}_{\boldsymbol{\theta}}^{(m)}, \boldsymbol{I}_{\boldsymbol{\varphi}}^{(m)}\right)$

$\boldsymbol{I}_{\boldsymbol{\theta}}^{(i)}=\left(E \eta_{i t}^{4}-1\right) \boldsymbol{J}_{\boldsymbol{\theta}}^{(i)} \quad$ and $\quad \boldsymbol{I}_{\varphi}^{(i)}=2 \boldsymbol{J}_{\varphi}^{(i)}$.

\section{Acknowledgments}

The authors thank the participants of the CFE 2016 conference in Seville, of the 8th French Econometric Conference at ESSEC Business School, of the 18th OxMetrics User Conference at CASS Business School, the 70th ESEM meeting in Lisbon and in particular Kris Boudt, Lynda Khalaf, Siem Jan Koopman, Marc Paolella and Andrew Patton for useful comments. The first author gratefully acknowledges the financial support of the chair QuantValley/Risk Foundation "Quantitative Management Initiative". The first two authors are grateful to the Agence Nationale de la Recherche (ANR), which supported this work via the Project MultiRisk (ANR-16CE26-0015-02). The second author also thanks the labex ECODEC.

\section{References}

Alexander, C., 2001. Orthogonal GARCH in mastering risk. Master. Risk 2, 21-38. Alexander, C., Chibumba, A.M., 1997. Multivariate Orthogonal Factor GARCH. Mimeo: University of Sussex, UK.

Amado, C., Teräsvirta, T., 2014. Conditional correlation models of autoregressive conditional heteroscedasticity with nonstationary GARCH equations. J. Bus. Econom. Statist. 32, 69-87.

Aue, A., Hörmann, S., Horváth, L., Reimherr, M., 2009. Break detection in the covariance structure of multivariate time series models. Ann. Statist. 37, 4046-4087.

Avarucci, M., Beutner, E., Zaffaroni, P., 2013. On moment conditions for quasimaximum likelihood estimation of multivariate ARCH models. Econometric Theory 29, 545-566.

Bauwens, L., Hafner, C., Laurent, S., 2012. Volatility models. In: Bauwens, L., Hafner, C., Laurent, S. (Eds.), Handbook of Volatility Models and Their Applications. John Wiley \& Sons, Inc., Hoboken, NJ, USA.

Bauwens, L., Laurent, S., Rombouts, J.V.K., 2006. Multivariate GARCH models: A survey. J. Appl. Econometrics 21, 79-109.

Berkes, I., Horváth, L., Kokoszka, P., 2003. GARCH processes: Structure and estimation. Bernoulli 9, 201-227.

Billingsley, P., 1961. The Lindeberg-Levy theorem for martingales. Proc. Amer. Math. Soc. $12,788-792$

Blasques, F., Gorgi, P., Koopman, S.J., Wintenberger, O., 2016. Feasible Invertibility Conditions and Maximum Likelihood Estimation for Observation-Driven Models, Tinbergen Institute Discussion Paper. 16-082/III.

Bollerslev, T., 1990. Modelling the coherence in short-run nominal exchange rates: A multivariate generalized ARCH model. Rev. Econ. Stat. 72, 498-505.

Bollerslev, T., Engle, R.F., Wooldridge, J.M., 1998. A capital asset pricing model with time-varying covariances. J. Polit. Econ. 96, 116-131.

Boswijk, H.P., van der Weide, R., 2011. Method of moments estimation of GO-GARCH models. J. Econometrics 163, 118-126.

Boudt, K., Laurent, S., Lunde, A., Quaedvlieg, R., Sauri, O., 2017. Positive semidefinite integrated covariance estimation, factorizations and asynchronicity. J. Econometrics 196, 347-367.

Bougerol, P., Picard, N., 1992a. Strict stationarity of generalized autoregressive processes. Ann. Probab. 20, 1714-1729.

Bougerol, P., Picard, N., 1992b. Stationarity of GARCH processes and of some nonnegative time series. J. Econometrics 52, 115-127.

Chan, K.S., 1993. Consistency and limiting distribution of the LSE of a TAR. Ann. Statist. 21, 520-533.

Comte, F., Lieberman, O., 2003. Asymptotic theory for multivariate GARCH processes. J. Multivariate Anal. 84, 61-84.

Doornik, J., 2012. Object-Oriented Matrix Programming Using Ox. Timberlake Consultants Press.

Engle, R.F., 2016. Dynamic conditional beta. J. Financ. Econ. 14, 643-667.

Fama, E., French, K., 1992. The cross-section of expected returns. J. Finance 47, 427-465.

Fama, E., French, K., 2004. The capital asset pricing model: Theory and evidences. J. Econ. Perspect. 18, 25-46.

Fan, J., Wang, M., Yao, Q., 2008. Modelling multivariate volatilities via conditionally uncorrelated components. J. R. Stat. Soc. Ser. B Stat. Methodol. 70, 679-702.

Francq, C., Thieu, L.Q., 2015. QML Inference for Volatility Models with Covariates. MPRA preprint No. 63198.

Francq, C., Zakoïan, J.-M., 2010. GARCH Models: Structure, Statistical Inference and Financial Applications. John Wiley. 
Francq, C., Zakoïan, J.-M., 2012. QML estimation of a class of multivariate asymmetric GARCH models. Econometric Theory 28, 179-206.

Francq, C., Zakoïan, J.-M., 2016. Estimating multivariate volatility models equation by equation. J. R. Stat. Soc. Ser. B Stat. Methodol. 78, 613-635.

Francq, C., Zakoïan, J.-M., 2017. Estimation Risk for the Var of Portfolios Driven by Semi-Parametric Multivariate Models. Preprint.

Glosten, L.R., Jaganathan, R., Runkle, D., 1993. On the relation between the expected values and the volatility of the nominal excess return on stocks. J. Finance 48 , 1779-1801.

Hamadeh, T., Zakoïan, J.-M., 2011. Asymptotic properties of LS and QML estimators for a class of nonlinear GARCH processes. J. Statist. Plann. Inference 141 488-507.

Hansen, P.R., Lunde, A., Nason, J.M., 2011. The model confidence set. Econometrica 79, 453-497.

Hansen, P.R., Lunde, A., Voev, V., 2014. Realized beta GARCH: Multivariate GARCH model with realized measures of volatility and covolatility. J. Appl. Econometrics 29, 774-799.

Harvey, A., 2013. Dynamic Models for Volatility and Heavy Tails: With Applications to Financial and Economic Time Series. Cambridge University Press, New York.

He, C., Teräsvirta, T., 2004. An extended constant conditional correlation GARCH model and its fourth-moment structure. Econometric Theory 20, 904-926.

Jeantheau, T., 1998. Strong consistency of estimators for multivariate ARCH models. Econometric Theory 14, 70-86.

Lanne, M., Saikkonen, P., 2007. A multivariate generalized orthogonal factor GARCH model. J. Bus. Econom. Statist. 25, 61-75.

Laurent, S., Rombouts, J., Violante, F., 2012. On the forecasting accuracy of multivariate GARCH models. J. Appl. Econometrics 12/6, 934-955.

Ling, S., McAleer, M., 2003. Asymptotic theory for a vector ARMA-GARCH model. Econometric Theory 19, 280-310.

Maheu, J.M., Shamsi, A.Z., 2016. Nonparametric Dynamic Conditional Beta. MPRA Paper No. 73764
Markowitz, H., 1952. Porfolio selection. J. Finance 7, 77-91.

McAleer, M., Hoti, S., Chan, F., 2009. Structure and asymptotic theory for multivariate asymmetric conditional volatility. Econometric Rev. 28, 422-440.

Nelson, D.B., 1991. Conditional heteroskedasticity in asset returns: A new approach. Econometrica 59 (2), 347-370.

Noureldin, D., Shephard, N., Sheppard, K., 2014. Multivariate rotated ARCH models. J. Econometrics 179, 16-30.

Osiewalski, J., Pajor, A., 2009. Bayesian analysis for hybrid MSF-SBEKK models of multivariate volatility. Cent. Eur. J. Econ. Model. Econ. 1, 179-202.

Patton, A., Verardo, M., 2012. Does beta move with news? firm-specific information flows and learning about profitability. Rev. Financ. Stud. 25, 2789-2839.

Pedersen, R.S., 2017. Inference and testing on the boundary in extended constant conditional correlation GARCH models. J. Econometrics 196, 23-36.

Pourahmadi, M., 1999. Joint mean-covariance models with applications to longitudinal data: Unconstrained parametrization. Biometrika 86, 677-690.

Sharpe, W., 1964. Capital asset prices: a theory of market equilibrium under conditions of risk. J. Finance 19, 425-442.

Sharpe, W., 1992. Management style and performance measurement. J. Portfolio Manag. 18, 7-19.

Silvennoinen, A., Teräsvirta, T., 2009. Multivariate GARCH models. In: Andersen, T.G., Davis, R.A., Kreiss, J.-P., Mikosch, T. (Eds.), Handbook of Financial Time Series. Springer, New York.

Tsay, R.S., 2010. Analysis of Financial Time Series, third ed. John Wiley \& Sons.

van der Weide, R., 2002. GO-GARCH: A multivariate generalized orthogonal GARCH model. J. Appl. Econometrics 17, 549-564.

Wald, A., 1949. Note on the consistency of the maximum likelihood estimate. Ann. Math. Stat. 20, 595-601.

Wintenberger, O., 2013. Continuous invertibility and stable QML estimation of the EGARCH(1,1) model. Scand. J. Stat. 40 (4), 846-867. 